\title{
Reach out and touch? : improving reach and use of an Internet-delivered lifestyle intervention
}

Citation for published version (APA):

Schneider, F. E. K. (2014). Reach out and touch? : improving reach and use of an Internet-delivered lifestyle intervention. [Doctoral Thesis, Maastricht University]. Uitgeverij BOXPress.

https://doi.org/10.26481/dis.20140131fs

Document status and date:

Published: 01/01/2014

DOI:

$10.26481 /$ dis.20140131fs

Document Version:

Publisher's PDF, also known as Version of record

\section{Please check the document version of this publication:}

- A submitted manuscript is the version of the article upon submission and before peer-review. There can be important differences between the submitted version and the official published version of record.

People interested in the research are advised to contact the author for the final version of the publication, or visit the DOI to the publisher's website.

- The final author version and the galley proof are versions of the publication after peer review.

- The final published version features the final layout of the paper including the volume, issue and page numbers.

Link to publication

\footnotetext{
General rights rights.

- You may freely distribute the URL identifying the publication in the public portal. please follow below link for the End User Agreement:

www.umlib.nl/taverne-license

Take down policy

If you believe that this document breaches copyright please contact us at:

repository@maastrichtuniversity.nl

providing details and we will investigate your claim.
}

Copyright and moral rights for the publications made accessible in the public portal are retained by the authors and/or other copyright owners and it is a condition of accessing publications that users recognise and abide by the legal requirements associated with these

- Users may download and print one copy of any publication from the public portal for the purpose of private study or research.

- You may not further distribute the material or use it for any profit-making activity or commercial gain

If the publication is distributed under the terms of Article $25 \mathrm{fa}$ of the Dutch Copyright Act, indicated by the "Taverne" license above, 


\section{Reach out and touch?}

Improving reach and use of an

Internet-delivered lifestyle intervention 


\section{COLOPHON}

Lay-out: Francine Schneider

Cover design and production: Proefschriftmaken.nl || Uitgeverij BOXPress

(C) Francine Elisabeth Karel Schneider

ISBN: 978-90-8891-784-4

The research in this thesis was conducted at the School for Public Health and Primary Care: CAPHRI, Department of Health Promotion, Maastricht University. CAPHRI participates in the Netherlands School of Primary Care Research (CaRe). CAPHRI was classified as "excellent" by the external evaluation committee of leading international experts that reviewed CAPHRI in December 2010. The research presented in this dissertation was funded by the Netherlands Organization for Health Research and Development (ZonMw, Grant No. 120710004)

All rights are reserved. No part of this book may be reproduced or transmitted in any form or by any means, without the written permission from the author or, where appropriate, the publisher of the article. 


\section{Reach out and touch?}

Improving reach and use of an

Internet-delivered lifestyle intervention

\section{PROEFSCHRIFT}

Ter verkrijging van de graad van doctor

aan de Universiteit Maastricht,

op gezag van de Rector Magnificus,

Prof. dr. L.L.G. Soete

volgens het besluit van het College van Decanen

in het openbaar te verdedigen op

vrijdag 31 januari 2014 om 14:00 uur

\section{Francine Elisabeth Karel Schneider}

geboren op 3 mei 1982

Kerkrade (Nederland) 


\section{PROMOTER}

Prof. dr. Hein de Vries

\section{COPROMOTER}

Dr. Liesbeth A. D. M. van Osch

\section{BEOORDELINGSCOMMISSIE}

Prof. dr. Nanne K. de Vries (voorzitter)

Prof. dr. Gerjo Kok

Prof. dr. Lilian Lechner, Open Universiteit Nederland

Dr. Anke Oenema

Prof. dr. Heleen Riper, Vrije Universiteit Amsterdam 
Contents 


\section{CHAPTER 1}

General introduction

\section{CHAPTER 2}

Optimizing diffusion of an online computer tailored lifestyle program: a study protocol

\section{CHAPTER 5}

The influence of user characteristics and a periodic email prompt on use of an Internetdelivered computer tailored lifestyle intervention

\section{CHAPTER 6}

Periodic email prompts

to re-use an Internetdelivered lifestyle program: influence of prompt content and timing
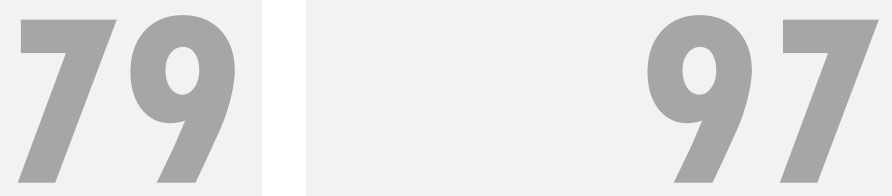


\section{CHAPTER 3}

The use of a proactive dissemination strategy to optimize reach of an Internet-delivered computer tailored lifestyle intervention

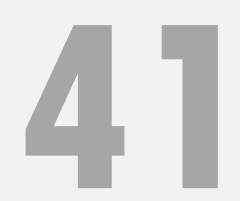

\section{CHAPTER 7}

General discussion

\section{CHAPTER 4}

Identifying factors for optimal development of health-related websites: a Delphi study among experts and potential future users

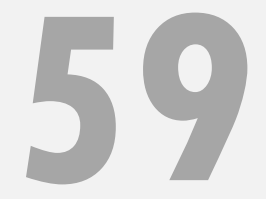

REFERENCES 135

APPENDICES 155

SUMMARY 165

SAMENVATTING 171

CURRICULUM VITAE 177

DANKWOORD 181 



\section{Chapter 1}

General introduction 

Chronic diseases, like cardiovascular diseases, different types of cancer and diabetes type II, are major causes of illness and death in the Netherlands as well as in other high income countries (RIVM, 2012; World Health Organisation, 2011). Important determinants of these diseases are partly related to lifestyle behaviours, like smoking, unhealthy eating habits, physical inactivity and excessive alcohol consumption. These behaviours are highly prevalent (RIVM, 2012; World Health Organisation, 2013) and often tend to co-occur (De Vries, Kremers, Smeets, Brug, \& Eijmael, 2008; Strecher, Wang, Derry, Wildenhaus, \& Johnson, 2002). Morbidity, mortality and prevalence of chronic diseases can be reduced by focussing on primary prevention and promoting the health of the population (World Health Organisation, 2013). Especially, health promoting initiatives that aim at motivating and assisting people to adopt or maintain a healthy lifestyle are essential (World Health Organisation, 2005).

\section{INTERNET-DELIVERED LIFESTYLE INTERVENTIONS}

The use of the Internet as a platform for the delivery of health promoting initiatives (i.e. interventions) offers substantial opportunities in terms of reach among the general public. Internet penetration rates are expanding rapidly, with currently almost two billion people having access to the Internet (Internet World Stats, 2013). Being at the forefront of this worldwide trend, Internet access in the Netherlands at this point covers 93\% of all inhabitants (CBS, 2012; Internet World Stats, 2013). Over recent years, adequacy of reach has also improved. Internet access among older people and people with a lower socioeconomic status has grown rapidly (Spittaels \& De Bourdeaudhuij, 2007; Tu \& Cohen, 2008), indicating gradual closing of the 'digital gap'. Besides high and broad levels of accessibility, the Internet also offers users a degree of anonymity, which might empower people to seek easily accessible help for a wide range of topics including physical activity, dietary control and more sensitive 
topics, like alcohol control and smoking cessation. These advantages have prompted health promoters in recent years to develop internet-delivered interventions. Internet-delivered interventions can be described as "typically behaviourally or cognitivebehaviourally based treatments that have been operationalized and transformed for delivery via the Internet. Usually, they are highly structured; self or semi-self-guided; based on effective face-to-face interventions; personalized to the user; interactive; enhanced by graphics, animations, audio, and possibly video; and tailored to provide follow-up and feedback" (Ritterband \& Thorndike, 2006). Seen from a developer's perspective, the Internet provides opportunities to deliver interventions that closely resemble face-to face interventions to an unlimited amount of people at relatively low costs (Griffiths, Lindenmeyer, Powell, Lowe, \& Thorogood, 2006). Internet-delivered interventions have been proven to be effective in changing a wide range of health behaviours, like physical activity (Norman et al., 2007; Van den Berg, Schoones, \& Vliet Vlieland, 2007; Vandelanotte, Spathonis, Eakin, \& Owen, 2007), dietary control (Norman et al., 2007; Weinstein, 2006), smoking cessation (Shahab \& McEwen, 2009) and alcohol control (White et al., 2010).

\section{REACH OF INTERNET-DELIVERED LIFESTYLE INTERVENTIONS}

At present, the empirical support for the effectiveness of Internet-delivered interventions has been demonstrated and is still emerging (Kohl, Crutzen, \& de Vries, 2013; Myung, McDonnell, Kazinets, Seo, \& Moskowitz, 2009; Portnoy, Scott-Sheldon, Johnson, \& Carey, 2008; Vandelanotte et al., 2007). However, even though the Internet has the potential to reach large amounts of people, efficacy trials have indicated that actual reach is failing to live up to the high expectations (De Nooijer et al., 2005; Glasgow et al., 2007; Leslie, Marshall, Owen, \& Bauman, 2005), with actual participation rates ranging from 2.5\% to 20\% (Brouwer et al., 2010; Glasgow et al., 2007; Leslie et al., 2005). Based on these findings, it is expected that reach and use of these interventions may be even less optimal when implemented in a real life setting (Evers, Cummins, Prochaska, \& Prochaska, 2005; Evers et al., 2003). In order to increase impact on public health of effective Internet-delivered interventions (De Vries \& Brug, 1999; Glasgow, Vogt, \& Boles, 1999), it is important to optimize reach and use of these interventions.

The RE-AIM framework distinguishes five dimensions that can be used to evaluate the public health impact of interventions: reach, efficacy, adoption, implementation and maintenance (Glasgow et al., 1999). Within this framework, reach is defined as 'an individual-level measure of participation involving the absolute number, proportion and representativeness of individuals who are willing to participate in the intervention'. Adoption, implementation and maintenance, originally aimed at the organi- 
sational level, can also be used to describe the degree of exposure to Internetdelivered interventions on an individual level (Crutzen, Kohl, \& de Vries, 2013). Bringing the Internet-delivered intervention to people's notice is an important first step and a prerequisite for actual use (Rogers, 1995). After becoming aware of the existence of the intervention, people must make an active decision to adopt the intervention. Adoption of the intervention can be regarded as accessing the intervention for a first time (first use) (Crutzen, 2009). People subsequently have to make a decision about the actual use of the intervention and about the degree to which they prefer to use it. In line with this decision process, implementation can be considered as the degree to which people use the interventions as intended (prolonged use). Finally, maintenance reflects the degree to which people revisit the intervention (sustained use) (Figure 1.1). The level of first time use of Internet-delivered interventions is generally low, with only small proportions of the potential target population actually accessing the intervention (Crutzen, 2009; Glasgow et al., 1999) and levels of prolonged use of the intervention as well as levels of sustained use of the intervention are even lower (Brouwer, Kroeze, et al., 2011).

\section{Figure 1.1. Graphic representation of first, prolonged and sustained use}

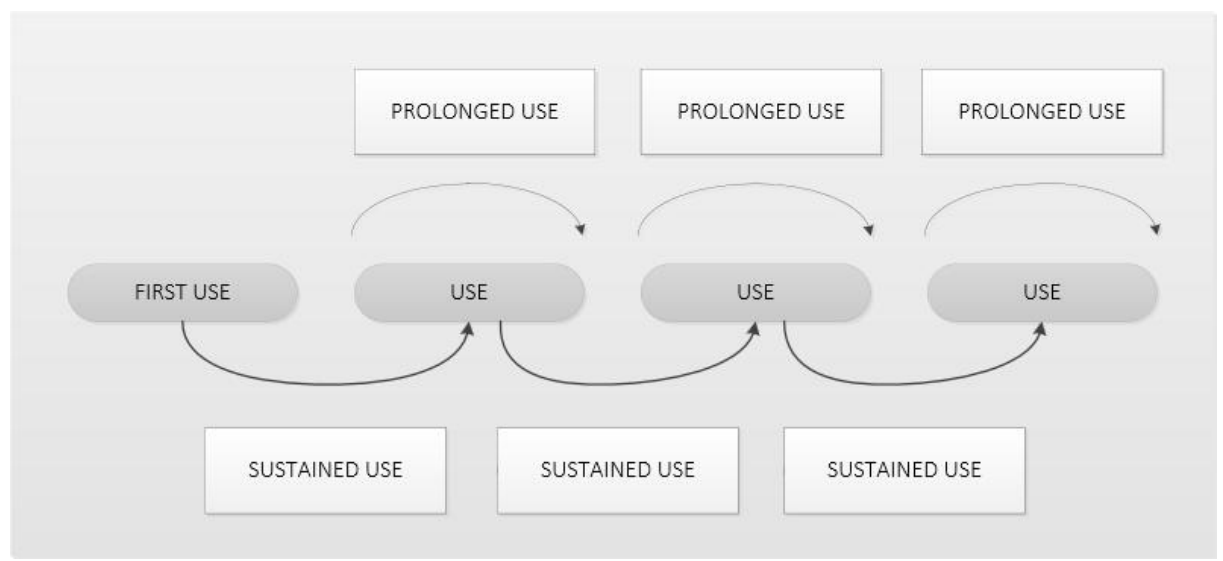

First use of Internet-delivered lifestyle interventions

First use of an Internet-delivered lifestyle intervention entails accessing the intervention for the first time. The Diffusion of Innovations Theory states that adoption of an Internet-delivered intervention is determined by several characteristics (Rogers, 1995). Firstly, intervention characteristics might have an influence on actual interven- 
tion use. Appearance of the intervention can have a substantial impact on its use (Ritterband, Thorndike, Cox, Kovatchev, \& Gonder-Frederick, 2009). Previous studies have for instance indicated that users prefer clearly structured websites with a simple design, that provide easy access, preferably without lengthy and complicated login procedures (Brouwer et al., 2008; Eysenbach \& Kohler, 2002).

Secondly, characteristics of the source delivering the intervention might have an influence on the adoption process. Studies have indicated that this source needs to be credible and reliable in order to proceed to actual intervention adoption (Brouwer et al., 2008; Brug, Oenema, Kroeze, \& Raat, 2005; Hong, 2006).

Thirdly, user characteristics might determine actual adoption of the intervention. Several steps are important in order for users to make a deliberate decision to visit an Internet-delivered intervention (Weinstein, 1988). First they must become aware of the existence of a health problem and their need for an (Internet-delivered) lifestyle intervention. After this, they must become engaged in the subject in order to make an active decision on using the intervention or not. However, research has indicated that people are often unaware of the seriousness of their symptoms (Blumenthal \& Endicott, 1998). Furthermore, other important barriers like lacking motivation to positively change one's behaviour (Ajzen, 1991), or insufficient knowledge on the existence of effective interventions might prevent large proportions of the population to actively seek assistance in changing their lifestyle behaviour (Ajzen, 1991; De Vries et al., 2003; Prochaska, Redding, \& Evers, 1997). In addition, several demographic characteristics, like socioeconomic status (SES), gender and age, can influence intervention use. First use of Internet-delivered lifestyle interventions is lagging behind among people with a lower SES (Lorence \& Park, 2008; Spittaels, De Bourdeaudhuij, Brug, \& Vandelanotte, 2007; Vandelanotte, De Bourdeaudhuij, Sallis, Spittaels, \& Brug, 2005; Yang, Lynch, Schulenberg, Roux, \& Raghunathan, 2008). Previous studies have also pointed out that Internet interventions tend to reach women (Brouwer et al., 2010; Etter, 2005; Spittaels, De Bourdeaudhuij, \& Vandelanotte, 2007) and older people (Steyaert \& de Haan, 2007).

Finally, the delivery strategy of the intervention has an impact on adoption. Dissemination of effective Internet-delivered interventions among the target population can occur in a reactive or proactive manner (Prochaska, Velicer, Fava, Rossi, \& Tsoh, 2001). The use of reactive dissemination strategies implies that a passive approach is taken in which users themselves must undertake action in order to benefit from the intervention content. This means that users must first become aware of their own need for a lifestyle intervention, after which they must actively search for a suitable intervention. However, insufficient knowledge, unawareness or lacking motivation of potential users currently hinders adoption of effective Internet-delivered interven- 
tions (De Vries et al., 2003; Prochaska, Redding, \& Evers, 1997). This stresses the necessity to invest in strategies through which lifestyle interventions can be proactively offered to the target population. These strategies have the potential to diminish important barriers for use and increase the knowledge of existing, evidence-based interventions (Velicer, Prochaska, Fava, Laforge, \& Rossi, 1999).

\section{Prolonged use of Internet-delivered lifestyle interventions}

After arriving at the intervention website for a first visit, it is important that people stay on the intervention for a sufficient amount of time in order to be exposed to the intervention, as this allows for notification of its content and involvement in all intervention components (Danaher, Boles, Akers, Gordon, \& Severson, 2006; Eysenbach, 2005; Strecher et al., 2008). Objective data can be collected in order to map the level of prolonged use. Several studies have reported on measures like the mean duration of each log on (Jacobs, De Bourdeaudhuij, \& Claes, 2010; Jansen et al., 2008; Leslie et al., 2005; Verheijden et al., 2004), often further specified by looking at average page view duration for each specific subsection of the intervention session (Lewis et al., 2008) or the surfing depth on the program website (Jacobs et al., 2010).

Several factors can have an influence on prolonged intervention use. According to the Model of Internet Interventions, the most proximal determinants of use are intervention and user characteristics (Ritterband et al., 2009). Website appearance is an important intervention characteristic that might influence prolonged use. Based on their first impression of the website, people make a decision to use or leave the website (Crutzen, De Kruif, \& De Vries, 2012). A previous study indicated that this process takes place immediately after arriving on the website, as results showed that the majority of visitors left the intervention website within 30 seconds (Brouwer et al., 2010). In addition to appearance, website content needs to be relevant and reliable (Brouwer et al., 2008; Ritterband et al., 2009). Previous studies have also indicated that tailoring website content to user characteristics can enhance prolonged use of Internet-delivered behaviour change programs (Brouwer et al., 2008; Couper et al., 2010; Strecher et al., 2008).

In addition, several user characteristics are known to have an influence on prolonged use. Previous studies have indicated that demographic user characteristics like age, gender and level of education are related to level of program engagement (Brouwer et al., 2010; Strecher et al., 2008; Verheijden, Jans, Hildebrandt, \& HopmanRock, 2007) with women, older and higher educated people displaying higher levels of program engagement. In addition, more cognitive user characteristics might influence prolonged use. People with higher levels of self-efficacy towards execution of the intended behaviour (Wangberg, Bergmo, \& Johnsen, 2008) are more likely to engage 
in the intervention content. Also, a positive attitude towards the health behaviour, motivational readiness to change and planning competences might positively influence on intervention use (De Vries et al., 2003; Ritterband et al., 2009; Van 't Riet, Crutzen, \& De Vries, 2010).

\section{Sustained use of Internet-delivered lifestyle interventions}

Over recent years, several studies have indicated that interventions consisting of a single intervention session can already have significant effects on behaviour change (Dijkstra, De Vries, \& Roijackers, 1998; Poel, Bolman, Reubsaet, \& de Vries, 2009). The impact of these interventions can be further augmented by providing intervention on several occasions (Borland, Balmford, \& Hunt, 2004). For interventions consisting of multiple sessions, a strong dose-response relationship exists between intervention use and behavioural effects (Crutzen, de Nooijer, Candel, \& de Vries, 2008; Verheijden et al., 2007). Sustained use of interventions must therefore be stimulated to further maximize their effects on health behaviour change (Christensen, Griffiths, \& Farrer, 2009; Norman et al., 2007).

Sustained use is an important issue, which has gained considerable attention during the last years. Eysenbach, addressing to this problem with his 'Law of Attrition', defines a clear distinction between drop-out attrition and non-usage attrition (Eysenbach, 2005). Where drop-out attrition refers to the process of losing participants to follow-up in research trials, non-usage attrition refers to the process of participants refraining from sustained use of the intervention. The focus area of this thesis is mainly directed at non-usage attrition of Internet-delivered computer tailored interventions. Research has demonstrated an initial rapid decline in intervention use over the first few weeks (Bennett \& Glasgow, 2009; Eysenbach, 2005). After these weeks, participants often lose interest in the program or realize that it does not fully meet their wishes or expectations. Since, research suggests that suboptimal adherence to interventions is likely to lessen their effect (Verheijden et al., 2007) and that achieving health behaviour change is a complex and lengthy process requiring continuous guidance (Christensen et al., 2009), sustained use of interventions must be stimulated.

Previous studies have identified several characteristics that are related to sustained use of Internet-delivered interventions. With regard to user characteristics, intervention users tend to be female, middle-aged and highly educated (Brouwer et al., 2010). Furthermore, participants with an unhealthier lifestyle (Brouwer et al., 2010) and increased levels of self-efficacy towards the desired behaviour were more likely to re-visit the Internet-delivered intervention (Wangberg et al., 2008). In addition, several intervention characteristics can be identified, that contribute to in- 
creased levels of sustained use. Firstly, incorporating important behaviour change strategies, such as providing personalized feedback and facilitating goal setting, can enhance sustained use (Brouwer, Kroeze, et al., 2011). Furthermore, sustained use can be stimulated by offering visitors the opportunity to self-monitor their level of behaviour change and by regularly offering new and updated intervention content (Brouwer et al., 2009). The provision of support is also a factor that contributes to sustained use, especially peer and counsellor support (Brouwer et al., 2009; Kelders, Kok, Ossebaard, \& Van Gemert-Pijnen, 2012). Finally, the use of periodic email reminders can be an effective strategy to enhance sustained use of Internet-delivered intervention (Brouwer, Kroeze, et al., 2011; Fry \& Neff, 2009; Wangberg et al., 2008).

\section{FOCUS OF THIS THESIS}

Suboptimal reach and use of Internet-delivered interventions is currently limiting their impact on public health (De Nooijer et al., 2005; Evers et al., 2005; Evers et al., 2003; Glasgow et al., 2007; Leslie et al., 2005). The studies described in this dissertation were part of a greater project. The focus of this thesis was on optimizing reach and use of the intervention. The intervention used in this dissertation was an Internetdelivered computer tailored lifestyle intervention, which integrated content from interventions aimed at increasing smoking cessation, promoting the intake of fruit and vegetables, increasing the level of physical activity and reducing the consumption of alcohol (Brug, Steenhuis, van Assema, Glanz, \& de Vries, 1999; Poel et al., 2009; Smeets, Brug, \& de Vries, 2008; Van Keulen et al., 2011). Studies aimed at establishing efficacy of the intervention were incorporated in the second part of the project (Schulz et al., 2011) and are therefore not included in this thesis.

\section{First use - a proactive dissemination strategy}

This project aimed at increasing first use of the intervention by using a proactive dissemination strategy. The Internet-delivered lifestyle intervention was embedded in an already existing channel; the Adult Health Monitor used by all Dutch Regional Public Health Services (RHPS).

In the Netherlands, RPHS's are responsible for the protection of inhabitants against diseases and calamity and for the promotion of health. In addition, all RPHS'S have a statutory obligation to periodically monitor the health of the adult population. By means of a nationwide survey, the Adult Health Monitor (AHM), the general health state of all Dutch adult inhabitants (aged 17 to 65) is assessed (GGD Nederland, 2011). This monitor takes place with an interval of four years and general health is assessed by inviting a representative sample of the adult population to complete a written or online version of the monitor questionnaire. Based on findings of this monitoring tool, 
future health policies at a national, provincial and local level are outlined. Overall levels of health, as well as different aspects of general health, e.g. physical, mental and social health, are assessed. This also includes the monitoring of important lifestyle behaviours, like physical activity, fruit and vegetable intake, smoking and alcohol consumption, according to guidelines set for these lifestyle behaviours (RIVM, 2005, 2012).

Within the current project, the Internet-delivered computer tailored lifestyle intervention was embedded in the AHM. After completion of the AHM questionnaire, all online participants were introduced to the intervention and were invited to use the intervention. Offering the lifestyle intervention as an additional service to AHM participants was expected to have several benefits. Firstly, embedding the intervention in the AHM might provide an important access point for reaching a considerably large segment of the Dutch population with an Internet-delivered lifestyle intervention. Secondly, by integrating the intervention in the AHM and offering the intervention to all online participants, awareness of the existence of the program was expected to increase. Thirdly, by embedding the intervention in an existing context, AHM data could directly be obtained and presented in order to inform people on the status of their current lifestyle. This was expected to increase the level of lifestyle awareness and serve as an important cue to action for participants included in the AHM to positively change their current lifestyle. Fourthly, integrating the Internet-delivered computer tailored lifestyle intervention may foster the RPHS's to optimize their public health education task; besides solely monitoring health behaviour, the RPHS's would now obtain an opportunity to provide people with personalized advice on how to change their lifestyle status. Finally, since the embedded intervention offered an overview of participants' current lifestyle status, as well as free of charge personalized advice and strategies on how to improve their lifestyle behaviours, the program was expected to serve as an incentive for online participation.

\section{Prolonged use - development and monitoring of an Internet-delivered lifestyle interven- tion}

The current project aimed at optimizing intervention development, as this is known to enhance positive users experiences, which subsequently result in prolonged intervention use (Crutzen, Cyr, \& de Vries, 2011). During the development process primary characteristics of an intervention are determined. According to Diffusion of Innovations Theory and Social Marketing principles (Cugelman, Thelwall, \& Dawes, 2011; Rogers, 1995), program development is one of the main processes to influence use of a new product (e.g. an Internet-delivered intervention). Besides including experts in the development process, development should also occur in accordance with needs 
and wishes of the target group (Cugelman et al., 2011). By considering and incorporating the opinion of potential future users, involvement is expected to increase, consequently resulting in increased appreciation of the intervention, lower levels of nonusage attrition and higher levels of prolonged and sustained use (Glasgow, Kleges, Dzewaltowski, Bull, \& Estabrooks, 2004). Development of the Internet-delivered computer tailored lifestyle intervention content and its online environment was guided by results from the Delphi study (step 1). By means of a Delphi study factors potentially related to optimal development of health-related websites were identified and agreed upon by experts and potential users. This resulted in a first version of the intervention. This version was further refined by conducting focus group interviews with potential futures users $(\mathrm{N}=20)$ using a think-aloud approach (step 2). These focus group interviews resulted in valuable insights with regard to experiences of users and their opinions about intervention content, appeal and ease of use. These insights were further complemented by results from a process-evaluation ( $N=521)$, which provided valuable information with regard to relevance of the intervention content, ease of use and satisfaction with the intervention content (step 3). Only results from the first step, entailing the Delphi study, are discussed in this thesis.

This intervention used a computer tailored approach, since tailoring website content to user characteristics is known to positively impact prolonged use of Internet-delivered interventions (Brouwer et al., 2008; Couper et al., 2010; Strecher et al., 2008). Over the recent years, positive effects of Internet-delivered lifestyle interventions applying computer tailored techniques have been reported (Krebs, Prochaska, \& Rossi, 2010; Lustria, Cortese, Noar, \& Glueckauf, 2009; Ritterband et al., 2003). Computer tailoring is a technique that is used to adapt intervention content to specific characteristics and circumstances of individuals using the intervention (De Vries \& Brug, 1999; Kreuter, Farrell, Olevitch, \& Brennan, 2000). The advantage of computer tailoring is that it allows for reaching large amounts of people with personalized health information (De Vries \& Brug, 1999). As a result, the provided information is more likely to be read, remembered and perceived as personally relevant, which results in intensified elaboration of this information and increased use of the intervention (Kreuter et al., 2000).

The Internet-delivered computer tailored lifestyle intervention integrated content of established programs that were tested and proven to be effective in randomized control trials for increasing smoking cessation, promoting the intake of fruit and vegetables, increasing the level of physical activity and reducing the consumption of alcohol (Brug et al., 1999; Poel et al., 2009; Smeets et al., 2008; Van Keulen et al., 2011). The intervention used a dual approach to guide people towards behaviour change. The first part consisted of a health risk appraisal and was aimed at increasing partici- 
pants' awareness of their current health behaviour status, by comparing their status to the Dutch public health guidelines set for these health behaviours (i.e. being moderately physically active for 30 minutes at least five days a week, eating two pieces of fruit per day, eating 200 grams of vegetables per day, not drinking more than one (women) or two (men) glasses of alcohol a day and non-smoking). Within this health risk appraisal, feedback messages were used to inform people on the current status of the health behaviour and to provide them with additional information on the content of the separate guidelines. In case of discrepancies between current behaviour and the guidelines, people were alerted and directed to the CT modules. Second, assistance was provided in changing participants' health behaviour by offering five separate computer tailored modules. The content of these modules was based on the Integrated Model for exploring motivational and behavioural change (I-Change Model) (Cremers, Mercken, Oenema, \& de Vries, 2012; De Vries et al., 2003; Elfeddali, Bolman, \& de Vries, 2012; Schulz et al., 2011). Within the modules, all health advices were adapted to individuals' characteristics by considering demographic, behavioural and cognitive characteristics (De Vries \& Brug, 1999).

Since prolonged use of the intervention is an important predictor of sustained use (Couper et al., 2010), it is important to track visitors' intervention use (Crutzen, Roosjen, \& Poelman, 2012) and to study determinants of prolonged use (Brouwer et al., 2010). This knowledge can subsequently be used to make specific program adjustments, or to develop specific strategies that can be used to enhance prolonged use of the program. Within the current project, prolonged use was closely monitored by studying levels of module initiation and completion and by studying predictors of initiation and completion.

\section{Sustained use - the use of periodic email prompts}

Currently Internet-delivered computer tailored lifestyle intervention are offered reactively to the public, implying a passive approach in which users themselves must undertake action in order to repeatedly use the intervention (Prochaska et al., 2001). Prior research has, however, demonstrated that attaining visitors' loyalty to an intervention over an extended period of time is a very effortful process, entailing continuous endeavours to keep users satisfied and interested (Eysenbach, 2005; Glasgow et al., 2007; Verheijden et al., 2007). Results from a recent review demonstrate the advantages of using periodic prompts as a relatively cost-effective method to encourage sustained use of lifestyle interventions (Fry \& Neff, 2009). Periodic email prompts can be used to invite people to re-use the intervention in order to self-monitor their level of behaviour change and to benefit from new and updated intervention content (Brouwer et al., 2009). 
However, most studies included in this review did not employ the use of a control group and long-term data collection. Therefore, to date, the effectiveness of periodic prompting cannot be firmly established. Additional research to reliably assess the effectiveness of proactive strategies, such as prompting, is therefore needed. Within this thesis we tested the effect of using periodic email prompt to increase use of the Internet-delivered lifestyle program. Furthermore, we examined optimal content and timing of email prompts to further optimize their effect.

\section{CONTENT OF THIS THESIS}

This thesis consists of three major parts, each consisting of several studies describing dissemination and adoption of the Internet-delivered computer tailored lifestyle program (first use: part 1), development of the intervention and intervention use (prolonged use: part 2) and attempts to increase adherence to the intervention (sustained use: part 3). The intervention and accompanied studies are registered in the Dutch Trial Register (NTR1786) and Medical Ethics Committee of Maastricht University and the University Hospital Maastricht (NL2723506809/MEC0903016).

\section{Part 1 - first use}

In the first part, the proactive dissemination strategy that was used in order to increase reach and use of the Internet-delivered intervention is described. First the Internet-delivered computer tailored lifestyle program, the proactive dissemination strategy and the study aims are described in a research protocol (chapter 2). Finally, in chapter 3 the results concerning reach of the dissemination strategy as well as detailed profiles of users and non-users and predictors of use of the Internet-delivered computer tailored lifestyle program are described.

\section{Part 2 - prolonged use}

In the second part qualitative and quantitative strategies are used to guide development of the intervention in close collaboration with users and experts. Results of a Delphi study among international experts and potential users conducted in order to distinguishing factors that might be related to optimal development of health related websites are described in chapter 4 . In addition, in chapter 5 an observational study is presented, regarding demographics, behavioural and socio-cognitive correlates of use of the Internet-delivered computer tailored lifestyle intervention.

\section{Part 3 - sustained use}

In the third part the results of using a proactive strategy consisting of periodic email prompts to boost sustained use of the Internet-delivered computer tailored lifestyle 
program are described. Chapter 6 studies the influence of content and timing of a single email prompt on sustained use of the Internet-delivered computer tailored lifestyle intervention. Finally, chapter 7 provides a general discussion of the main findings of this thesis and considers suggestions for future research, as well as important implications for theory and practice. 


\section{Chapter 2}

\section{Optimizing diffusion of an online}

computer tailored lifestyle program: a study protocol 


\section{ABSTRACT}

Although the Internet is a promising medium to offer lifestyle interventions to large amounts of people at relatively low costs and effort, actual exposure rates of these interventions fail to meet the high expectations. Since public health impact of interventions is determined by intervention efficacy and level of exposure to the intervention, it is imperative to put effort in optimal dissemination. The present project attempts to optimize the dissemination process of a new online computer tailored generic lifestyle program by carefully studying the adoption process and developing a strategy to achieve sustained use of the program. A prospective study will be conducted to yield relevant information concerning the adoption process by studying the level of adoption of the program, determinants involved in adoption and characteristics of adopters and non-adopters as well as satisfied and unsatisfied users. Furthermore, a randomized control trial will be conducted to the test the effectiveness of a proactive strategy using periodic email prompts in optimizing sustained use of the new program. Closely mapping the adoption process will gain insight in characteristics of adopters and nonadopters and satisfied and unsatisfied users. This insight can be used to further optimize the program by making it more suitable for a wider range of users, or to develop adjusted interventions to attract subgroups of users that are not reached or satisfied with the initial intervention. Furthermore, by studying the effect of a proactive strategy using period prompts compared to a reactive strategy to stimulate sustained use of the intervention and, possibly, behaviour change, specific recommendations on the use and the application of prompts in online lifestyle interventions can be developed. 


\section{INTRODUCTION}

Due to its high accessibility and its potential to reach large numbers of people, the Internet is considered to be a promising medium in the field of health promotion for offering tailored and targeted behaviour change programs to the general public (Bansil, Keenan, Zlot, \& Gilliland, 2006; Brug et al., 2005; Internet World Stats, 2013; Ritterband et al., 2003; World Health Organisation, 2011). Over the recent years, positive effects of online interventions applying computer tailored (CT) techniques have been reported (Krebs et al., 2010; Lustria et al., 2009; Ritterband et al., 2003), addressing physical activity (Van den Berg et al., 2007; Vandelanotte et al., 2007), fruit and vegetable intake (Brug, Glanz, Van Assema, Kok, \& van Breukelen, 1998; Neville, O'Hara, \& Milat, 2009), smoking (Borland et al., 2004; Dijkstra, De Vries, \& Roijackers, 1998; Strecher, 1999) and alcohol consumption (Riper et al., 2008; White et al., 2010). However, in sharp contrast to these high promises, actual reach of these interventions is failing to live up to the high expectations (Cook, Heath, \& Thompson, 2000; Crutzen, 2009; De Nooijer et al., 2005; Evers et al., 2005; Eysenbach, 2005; Glasgow et al., 2007).

Since public health impact of interventions is determined by their efficacy and exposure in the target group (De Vries \& Brug, 1999; Glasgow et al., 1999), it is important to optimize exposure to these interventions by conducting implementation studies and optimizing the methods by which they are disseminated to the general public. Earlier studies defined exposure as pertaining to three different aspects; accessing the intervention (first use), staying on the intervention for a substantial period of time (prolonged use) and revisiting the intervention (sustained use) (Crutzen, 2009). Adop- 
tion, or first use, rates of online interventions are generally considered to be low (Crutzen, 2009; Glasgow et al., 1999; Rogers, 1995), causing actual levels of engagement in the intervention as well as adherence to the intervention to be even lower. Since health behaviour change is a complex process, achieving sustained behaviour change is not only dependent on adoption of a behaviour change program. During a visit, intensive engagement in the program is essential to allow further notification of its content and involvement in its effective components (Danaher et al., 2006; Eysenbach, 2005). Furthermore, the number of visits to an intervention is important. Due to a high dose-response relation (Crutzen, de Nooijer, Candel, et al., 2008; Verheijden et al., 2007), sustained use of the program is essential to further maximize its effect on subsequent health behaviour change (Christensen et al., 2009; Norman et al., 2007).

The current project aims at optimizing exposure rates of a combination of several effective online CT programs, targeting physical activity, fruit and vegetable consumption, smoking and alcohol consumption, offered through the Internet. This combined $\mathrm{CT}$ program will be integrated in the Dutch Adult Health Monitor, a monitoring tool used by all Regional Public Health Service (RPHS) in the Netherlands in order to assess different aspects of general health and health related topics at fixed points in time. This integration will result in an online program that respondents of the Dutch Adult Health Monitor can turn to in order to obtain detailed and personalized information regarding their current health behaviour status, as well as personalized advice on how to improve their health by focusing on the key health behaviours.

Several theories underline the importance of different processes to foster effective diffusion of interventions (Cugelman et al., 2011; Rogers, 1995). These processes pertain to actual adoption (or first use) of the intervention by the target group and development of strategies to achieve sustained use of the intervention over time. In order to increase adoption rates of an intervention, it is imperative to obtain detailed profiles of those who successfully adopt a new intervention, i.e. so-called innovators or early adopters (Glasgow et al., 1999; Rogers, 1995). It is important to reach those people that benefit most from online lifestyle interventions, i.e. the people that engage in risk behaviours like smoking, excessive alcohol use, lack of physical activity or unhealthy eating patterns. By studying characteristics of first-time users and mapping the way the program is used and re-used, detailed knowledge on who adopts the intervention and on determinants of adoption can be obtained. Subsequently, this knowledge can be used to further optimize intervention content, as well as dissemination strategies in order to reach the target group. Furthermore, if first use is established, effective strategies need to be developed and tested in order to attain sustained use by first time users. 
Currently computer tailored interventions are offered reactively to the public, implying a passive approach in which users themselves must undertake action in order to benefit from the intervention (Prochaska et al., 2001). Prior research has, however, demonstrated that attaining visitors' loyalty to an intervention over an extended period of time is a very effortful process, entailing continuous endeavours to keep users satisfied and interested (Eysenbach, 2005; Glasgow et al., 2007; Verheijden et al., 2007). The suboptimal levels of exposure that are currently reached with the use of reactive strategies, combined with the high levels of attrition, indicate that the efforts put into ensuring visitor's loyalty are inadequate (Danaher et al., 2006). Therefore, focus should be directed towards developing and testing more proactive approaches in order to increase sustained use of effective behaviour change programs. Results from a recent review demonstrate the advantages of using periodic prompts as a relatively cost-effective method to encourage involvement in health promoting interventions (Fry \& Neff, 2009). However, since most studies included in this review refrained from using control groups and long-term data collection, to date, effectiveness of periodic prompting cannot be firmly established. Additional research to reliably assess the effectiveness of proactive strategies, such as prompting, is therefore needed.

In order to optimally disseminate the program, the present research project will thoroughly investigate adoption of an online CT lifestyle program and development of strategies to achieve sustained use. First, studying the adoption process will yield results on the level of adoption, determinants involved in adoption and characteristics of adopters and non-adopters as well as satisfied and unsatisfied users. A strategy aimed at increased and sustained level of use will be designed and tested. On that account the efficacy of a pro-active approach using periodic email prompts in order to obtain sustained use of the intervention over time will be studied in a randomized control trial.

\section{METHODS}

The current project emphasizes the processes of first use (adoption) and re-use of a combined computer tailored online lifestyle program, by examining each process in a single study. First, a general description of the CT program will be provided, before proceeding to the detailed description of the separate studies. The current project is approved by the Medical Ethics Committee of Maastricht University and the University Hospital Maastricht (NL27235.068.09/MEC 09-03-016) and is registered with the Dutch Trial Register (NTR1768). 


\section{The CT program}

The new CT program integrates content from established CT programs tested and proven to be effective in randomized control trials for increasing smoking cessation, promoting the intake of fruit and vegetables, increasing the level of physical activity and reducing the consumption of alcohol (Brug et al., 1999; Poel et al., 2009; Smeets et al., 2008; Van Keulen et al., 2008). The multi-component CT program is embedded in an already existing channel; the Adult Health Monitor used by all Dutch RPHS's. This combination results in an online CT program that a substantial proportion of the Dutch population can use in order to obtain detailed and personalized information regarding the status of their current health behaviours, as well as personalized advice on how to positively change their health by focusing on key determinants of the five health behaviours.

\section{The Adult Health Monitor}

The Adult Health Monitor takes place with an interval of four years and serves as a monitoring tool used by the RPHS's to assess the overall level of health in the Dutch population by approaching a representative sample of the population to fill out a questionnaire assessing different aspects of general health (e.g. physical and mental health) and health related topics (e.g. social and physical environment) (GGD Nederland, 2011). Physical health entails the five key behaviours targeted in this intervention; physical activity, measured by the Short QUestionnaire to ASsess Health enhancing physical activity (SQUASH) (Wendel Vos, Schuit, Saris, \& Kromhout, 2003), fruit and vegetable intake, measured by the Short Questionnaire for Fruit and Vegetable Intake (Bogers, Van Assema, Kester, Westerterp, \& Dagnelie, 2004; Van Assema, Brug, Ronda, Steenhuis, \& Oenema, 2002), alcohol consumption, measured by the Dutch Quantity-Frequency-Variability Questionnaire (QFV) (Lemmens, Tan, \& Knibbe, 1992) and smoking, measured by the abbreviated version of the Fagerström Test for Nicotine Dependence (Heatherton, Kozlowski, Frecker, \& Fagerstrom, 1991). Results obtained through this Adult Health Monitor are communicated to the concerning municipalities, which subsequently use these as a basis for future health policies.

Integrating the new CT program into the Adult Health Monitor may have several benefits. Firstly, it can enable the RPHS's to optimize their public health education task; besides solely monitoring health behaviour, the RPHS's now have an opportunity to provide people with personalized advice on how to change their health behaviours. Furthermore, it may provide the random sample of adults included in the Adult Health Monitor an incentive for their participation and might serve as a cue to action for them to positively change their health behaviour. Since the current CT program is embedded in the Adult Health Monitor of the Provinces of Limburg, Brabant and Zee- 
land, with an approximate total reach of 100.000 inhabitants, this integration also provides an important access point for reaching a considerably large segment of the Dutch population with a lifestyle intervention.

\section{Content of the CT program}

The CT program uses a step-by-step approach to guide people towards behaviour change. After online completion of the Adult Health Monitor people are informed in detail on the embedded program and the accompanied studies. People that indicate to be interested in the program are subsequently linked to the program. During their first visit demographic data (age, gender, height, weight, religious background, ethnicity, educational level and income) and personal data concerning the target health behaviours, acquired through the Adult Health Monitor questionnaire, are transferred to the CT program and used to inform people on the current status of their health behaviours by comparing these to Dutch public health guidelines (i.e. being moderately physically active for 30 minutes at least 5 days a week, eating 2 pieces of fruit per day, eating 200 grams of vegetables per day, not drinking more than one (women) or two (men) glasses of alcohol a day and non-smoking). In case of discrepancies, people are alerted and provided assistance in changing their health behaviour(s) with help of CT advices available per behaviour.

Changing health behaviours with help of computer tailoring is the main component of the program. A CT module is available per health behaviour and within each module people have the opportunity to obtain personalized advices regarding the opted health behaviour. All health behavioural advices are adapted to individuals' characteristics by considering demographic, behavioural and social-cognitive variables (De Vries \& Brug, 1999; Dijkstra \& De Vries, 1999; Kreuter et al., 2000). Some variables, such as individuals' current health behaviours and characteristics of the respondent (gender), are directly obtained through the Adult Health Monitor. Other variables, such as cognitive variables with regard to the health behaviour (attitude, perceived social influence and self-efficacy), intention to change the health behaviour and planning strategies (action plans and coping plans) are assessed by using an additional tailoring questionnaire. All tailoring components are based on the Integrated Model for exploring motivational and behavioural Change (I-Change Model) (De Vries et al., 2003).

\section{Intervention materials}

Message content and algorithms of the CT advices are based on prior theory and evidence based CT interventions on physical activity, fruit and vegetable intake, alcohol consumption and smoking cessation (Poel et al., 2009; Smeets et al., 2008; 
Smeets, Kremers, Brug, \& de Vries, 2007; Van Keulen et al., 2008). Online questionnaires available for the health behaviours serve as basis for the CT advices. Attitude is measured by investigating the respondent's level of agreement to a total of 6 statements regarding the pros ( 3 statements) and cons ( 3 statements) of the health behaviour under consideration (five-point scale; e.g. 'When I quit smoking, my health will improve', 'When I quit smoking, I will gain weight'). Social influence is measured by three different concepts; social norms, social modelling and social support. Social norms are assessed with one item, asking respondents to complete statements regarding perceived support from people in their environment (i.e., partner, family members, friends and colleagues) regarding the health behaviour (five-point scale; e.g. 'According to the people within my direct environment.... I certainly should not smoke'). Social modelling is assessed by one item asking participants how many people within their environment (i.e., partner, family members, friends and colleagues) engage in the health behaviour under consideration (five point scale; e.g. 'How many of your family members eat at least two pieces of fruit every day?'). Social support is measured with one item assessing the degree of support respondents receive from the people within their direct environment (i.e., partner, family members, friends and colleagues) to engage in the health behaviour (four-point scale; e.g. 'Do your friends stimulate you to be physically active?'). Self-efficacy is measured by means of 6 items asking participants to indicate whether they would be able to engage in (physical activity and nutrition) or refrain from (smoking and alcohol consumption) the key health behaviour when encountering difficult situations (seven-point scale; e.g. 'Will you be able to eat a sufficient daily amount of vegetables when other tempting alternatives are within reach?'). The intention to change behaviour is measured by means of two items. The first item is an extended version of the Stages of Change concept (Prochaska \& DiClemente, 1983; Prochaska et al., 1997) using an algorithm consisting of ten stages varying from unaware to action, whereas the second item assesses the degree to which people are considering to engage in the key behaviour in the future; (seven-point scale; e.g. 'Do you intend to quit smoking in the future?'). Action planning is measured by three items asking respondents to indicate whether they plan to use a particular preparatory strategy when engaging in a certain behaviour (five-point scale; e.g. 'Are you planning to join a sports club?'). Coping planning is assessed by six questions asking participants to what degree they have made a specific plan regarding how to cope with difficult situations that might hinder execution of the health behaviour. These questions are based on the difficult situations used for the self-efficacy items (five-point scale; e.g. 'I have made a detailed plan to refrain from smoking when I just finished a meal.'). 


\section{STUDY 1 - ASSESSING THE ADOPTION PROCESS OF THE CT PROGRAM}

The main goal of the first study is to assess the level of first time use of the new CT program, frequency of use, level of satisfaction with the new CT program by participants of the Adult Health Monitor 2009 and determinants of adoption and use. Furthermore, characteristics of users and non-users and satisfied and unsatisfied users will be investigated.

\section{Design}

This is a prospective study, employing a longitudinal design. At baseline participants of the Adult Health Monitor interested in the new CT program receive feedback concerning their current health behaviour. In case of a negative discrepancy between the status of their current health behaviours and public health guidelines set for these behaviours participants are stimulated to change these behaviours with help of the CT program. During a period of three months, use of the CT program is monitored anonymously resulting in a detailed overview of number of first visits, revisits, and the specific health behaviours selected.

\section{Recruitment and procedure}

The research sample consists of Dutch adults participating in the Adult Health Monitor 2009 of the Dutch Provinces of Limburg, Brabant and Zeeland. The RPHS's will invite a representative sample of inhabitants of the provinces North-Brabant, Zeeland and Limburg (+/- 100.000) by mail to participate in the Monitor. Participants will be offered the opportunity to fill out a written questionnaire, which is included, or to complete an online version of the Monitor questionnaire. Electronic completion will be highly encouraged since the new CT program is an online program only accessible for respondents participating via the Internet. Based on previous experiences with this Monitor it is expected that a response rate of approximately $33 \%$ will be reached, indicating that about 33.000 respondents will fill out the Adult Health Monitor.

After completing the Adult Health Monitor, all participants of the online version are introduced to the new embedded program, which is free of charge and consists of an opportunity to receive CT feedback about their health behaviour. Participants indicating to be interested in the new program will be asked to leave their email address and will receive an email including an invitation to log in to the CT program with a personal login code and password approximately three weeks after completion of the Monitor. After logging on to the program participants receive detailed information on the goal and the content of both studies described in this protocol. After receiving this information, participants are asked to fill out an online informed consent form. Only those participants that consent to participation are directed to the new service. Sub- 
sequently, data on demographics and the five health behaviours obtained through the Monitor are transported to the new program, resulting in a personal overview in which an individual's current health behaviour status is compared to the guidelines set for these health behaviours. Within the program, five modules generating CT health advice are available to assist participants in changing their current health behaviour status when needed. Once respondents decide to leave the CT program after their first visit, they will be asked to fill out an additional questionnaire to assess their satisfaction with the program and their intention to revisit the program again in the near future (Figure 2.1).

\section{Inclusion and exclusion criteria for participants}

Participants can be included in the current study when they are between 18 and 65 years and able to understand the Dutch language sufficiently. Moreover, they have to have access to the Internet. There are no explicit exclusion criteria stated for the current study.

\section{Measures}

User characteristics

User characteristics are collected to produce a detailed user profile entailing information on personal characteristics, health behaviour status as well as socio-cognitive characteristics. Questions used in the Health Monitor pertaining to personal characteristics, included questions on gender, height, weight, marital status, religious background, ethnicity, educational level, current work status, and income. Furthermore, questions used in the Health Monitor pertaining to physical activity (Wendel Vos et al., 2003), fruit and vegetable intake (Bogers et al., 2004), alcohol intake (Lemmens et al., 1992) and smoking behaviour (Heatherton et al., 1991) were used to assess health behaviour status. Finally, several determinants of health behaviour as mentioned in the I-Change model were used as socio-cognitive characteristics; intention, attitude, social influence, self-efficacy, action and coping planning.

\section{Program evaluation}

Satisfaction with the CT program will be assessed by using a total of five items; four items measuring the degree to which respondents regard the information provided within the program as new, personal, useful and encouraging (five-point scale; e.g. 'I think the information within the CT program is useful') and one item assessing overall satisfaction (ten-point scale; e.g. 'How satisfied are you with the current program?'). 


\section{Figure 2.1. Flowchart study 1}

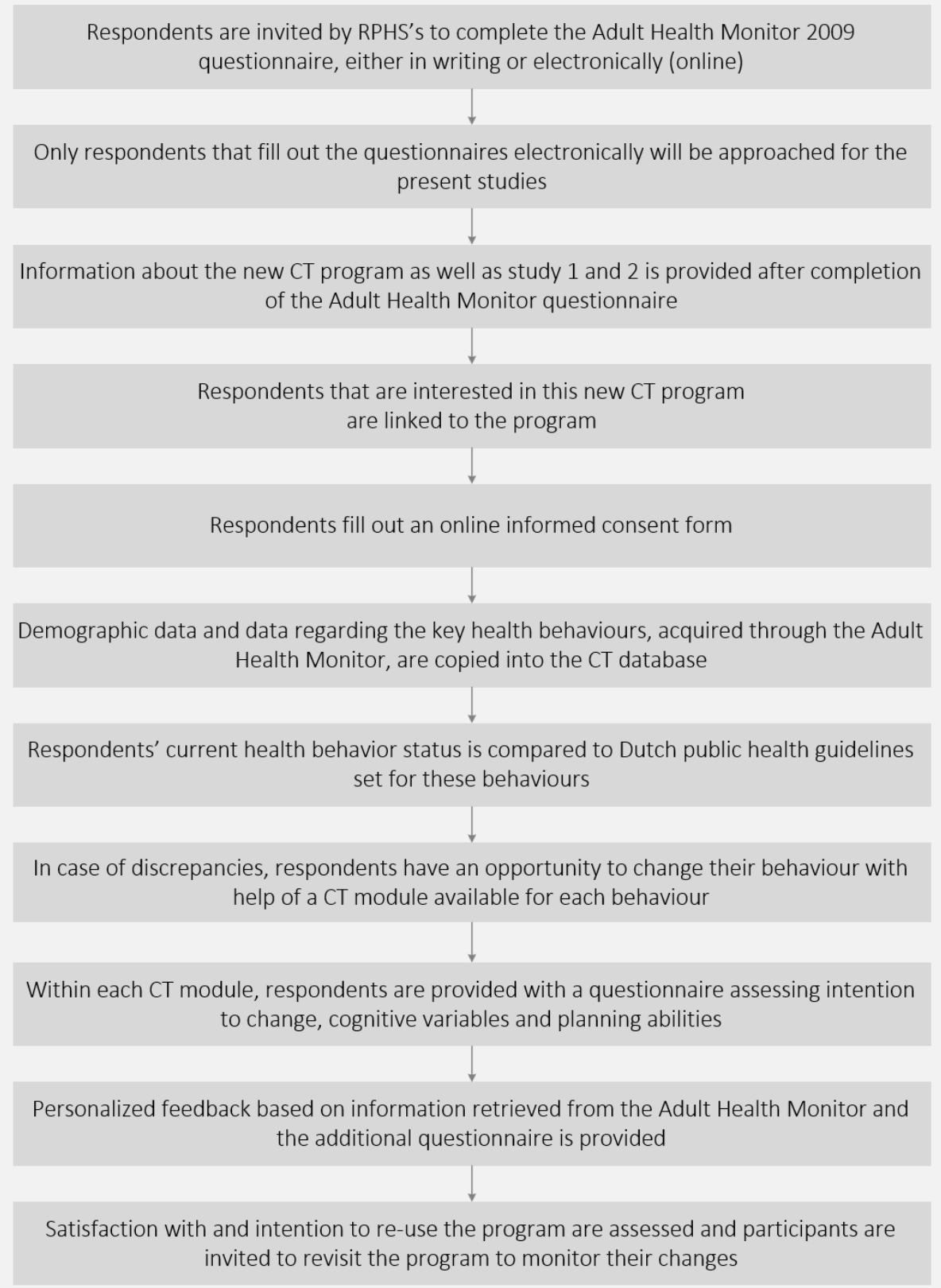


Intention to re-use the program

Intention to re-use the CT program is assessed by using a 10 point intention scale ('I intend to use this CT program again in the near future').

\section{Outcome measures}

To measure the adoption level of the new CT program focus will be on level of first use, demographic and behavioural characteristics of users and non-users, satisfaction with the program, characteristics of satisfied and unsatisfied users, intention to revisit the program and frequency of revisits. In order to measure level of first use of the program, two dichotomous variables will be created; one indicating whether participants initiate a behaviour change module and one indicating whether participants finish the module. Initiating a module is labelled as "yes" when people fill out the first question of this module, which is the question concerning intention to change the specific health behaviour. Completion of a module is labelled as "yes" when people also fill out the final question of the module. Re-visiting of the program will be assessed by monitoring whether participants log in to the program within a three month period after baseline ( 0 =yes $/ 1=$ no). Revisits to the program will be measured by comparing the dates of log-in to the baseline date.

\section{Statistical analysis}

First, general descriptive statistics will be used to describe personal characteristics of the participants, as well as main findings concerning current health behaviour status, adherence to the public health guidelines and socio-cognitive characteristics. Second, logistic regression analyses will be conducted with initiation of a module, completion of a module and revisiting of the program as dependent variables. Demographics, such as gender, height, weight, marital status, religious background, ethnicity, educational level, current work status, income, health behaviour status and cognitions (measured at baseline) are included in the model as predictors.

\section{STUDY 2 - INCREASING SUSTAINED USE OF THE PROGRAM}

The aim of this study is to test the effect of using a proactive approach (i.e. periodic email prompting) on revisiting of the program compared to a reactive approach (no additional email prompting). Furthermore, the efficacy of these approaches with regard to behaviour change will be studied.

\section{Design}

The second study consists of a randomized control trial (RCT) including one experimental group. A design with a baseline measurement and three follow-up measure- 
ments (6, 12 and 18 months) measuring health behaviour status, intention to change, cognitive variables and planning abilities, will be used to compare the experimental condition (use of a proactive technique) to a control condition (use of a reactive approach).

\section{Recruitment and procedure}

The research sample consists of individuals that participate in the Dutch Adult Health Monitor and that decide to pay a first visit to the new CT program. After logging in to the program at baseline and consenting to participation by filling out an online informed consent form, participants are randomly assigned to one of the two conditions. People in the experimental condition are reminded proactively via email every three months for a period of 18 months to visit the CT program in order to obtain iterative feedback concerning their health behaviour status and additional CT advice, whereas people in the control condition do not receive any additional prompts and are only encouraged to re-visit the program in the future at baseline. All participants will be invited by email for the follow-up measurements at 6, 12 and 18 months (Figure 2.2).

\section{Figure 2.2. Flowchart study 2}

All respondents participating in study 2 are randomly assigned to either the control or the experimental condition, by means of a computer software randomization device

\section{Prompting condition}

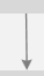

Participants are reminded proactively via email every three months that they can visit the new service as often as they want to fill out the behavioral questions and to receive feedback on changes in behavior

\section{No prompting condition}

Participants are reminded reactively at baseline (no-prompts) that they can visit the new service as often as they want to fill out the behavioral questions and to receive feedback on changes in behavior

Follow-up measurements assessing health behavior status, intention to change, cognitive variables and planning abilities at 6,12 and 18 months 


\section{Inclusion and exclusion criteria for participants}

Participants can be included in the current study when they are between 18 and 65 years and able to understand the Dutch language sufficiently. Moreover, they have to have access to the Internet. There are no explicit exclusion criteria stated for the current study.

\section{Materials}

Three months after their first visit, participants in the experimental condition receive an email, prompting them to revisit the program. This email opens with a personal salutation and reminds people about their first visit to the program. Also, in short, the content and purpose of the program will be summarized, in case people have forgotten about the details of the program. Subsequently, people are invited to revisit the program in order to obtain information on their current health status and to monitor their progress. Participants were also pointed out the opportunity to receive additional, iterative, health advice on the health behaviour selected at baseline or on a new behaviour. In order to facilitate logging in to the program, the email will also contain details on personal log-in information (user name and password).

\section{Randomization}

Interested participants are directed through the Adult Health Monitor to the new program, where they can log in to the program with a personal username and password. After signing in to the program, participants will be randomly allocated to either the control condition or the experimental condition. Randomization will occur at the respondent level by means of a computer software randomization device. Blinding of participants is not possible, since participants will take notice of the fact that they receive additional email prompts or not.

\section{Outcome measures}

\section{Primary outcome measures}

The primary outcome measures are revisits to the program and level of behaviour change. Program revisits are objectively monitored for a total period of eighteen months. Recordings will be done anonymously and will yield information about the number of times the program is visited, the duration of each visit (in minutes) and which specific behaviours are most frequently selected. Level of behavioural change is assessed by studying participants' adherence to public health guidelines set for these behaviours ( 0 = not complying with recommendations $/ 1$ = complying with recommendations). 


\section{Secondary outcome measures}

Secondary outcome measures include overall tobacco use (number of cigarettes/day), level of physical activity (minutes/day), and overall level of fruit (pieces/day), vegetables (grams/day) and alcohol consumption (glasses/day). Furthermore, socio-cognitive measures, such as intention, attitude, social influence, self-efficacy, action and coping planning will be measured.

\section{Statistical analysis}

Sample size and power

With regard to revisits to the program (first primary outcome measure), it is estimated that approximately $50 \%$ of the respondents of the no-prompting condition will visit the site more often than four times (after the baseline, and the first three post-tests after six, twelve and eighteen months). It is estimated that in the prompting condition $70 \%$ of the respondents will visit the site more than four times. Power calculations reveal that in order to be able to detect this difference with a power of $80 \%(p=.05)$ at least 186 persons per condition are needed. Taking into account potential attrition, it is proposed to collect the data of 250 persons per cell. The second primary outcome measure is the level of health behaviour change. The current program entails a total of five health behaviours. Therefore, with regard to the assessment of behaviour change (second outcome measure), estimations for finding effects will be based on smoking, since this behaviour results in the most conservative power calculations. Approximately $25 \%$ to $30 \%$ of the population smoke (RIVM, 2008). Power estimations are based on the ability to detect a difference in quit rates of $10 \%$ with a power of .80 and a p-value of .05. It is estimated that, in order to detect this significant difference in quit rates, 219 smoking persons per cell are needed, resulting in a total of 438 smokers (DSS Research, 2011). To be able to have 438 smokers in the sample (under the condition that $30 \%$ of the Dutch population smokes), 1575 participants need to be recruited. Accounting for $35 \%$ attrition during the trial period, the total sample size for this study will be approximately 4500 persons.

\section{Analyses}

First, general descriptive statistics will be used to describe personal characteristics of the participants, as well as findings concerning current health behaviour status, adherence to the public health guidelines and socio-cognitive characteristics. Second, logistic regression analyses will be conducted with revisits to the program and level of behaviour change as dependent variables. Furthermore, linear regression analyses will be conducted with number of revisits, duration of revisits and number of behaviours selected as dependent variables. Demographics, such as gender, height, weight, mari- 
tal status, religious background, ethnicity, educational level, current work status, and income, health behaviour status and cognitions (measured at baseline) will be included in the model as predictors of the dependent variables.

\section{DISCUSSION}

Although the Internet is a promising medium to offer lifestyle interventions (Internet World Stats, 2013), it is not yet used to its full potential, since actual exposure to online lifestyle interventions is falling short to the high expectations (De Nooijer et al., 2005; Glasgow, 2007). Since public health impact of interventions is determined by efficacy and actual use of the intervention by the target group (Crutzen, 2009), it is necessary to ensure optimal reach of and exposure to these interventions. The current implementation project aims at optimizing the methods by which effective computer tailored programs are disseminated to the general public.

Since optimal dissemination largely depends on the processes of actual adoption of the intervention and development of a strategy to achieve sustained use of the intervention over time (Rogers, 1995), each process is thoroughly and independently studied. Firstly, closely mapping the adoption process of the intervention will gain insight on characteristics of adopters and non-adopters and satisfied and unsatisfied users, as well as determinants of adoption and use. This acquired knowledge can be used to optimize the existing intervention by making it more suitable for the intended target group of Dutch adults aged 18 to 65 years, or to develop adjusted interventions to attract certain subgroups of users that are not satisfied or not reached with the initial intervention. Furthermore, attracting users to the intervention multiple times allows for repeated exposure to the content of the intervention and assimilation of the effective components within the intervention, resulting in an increased likelihood of actual behaviour change. Therefore, focus within the current project is also directed at developing and testing a proactive strategy that may be used to increase sustained use of the intervention over time. Findings from this study are relevant for the current program, but also for other existing and future interventions. Even though a proactive strategy is a relatively simple method and therefore often used in current online health promoting interventions, little is known about their positive or even adverse effects (Fry \& Neff, 2009). Therefore, the current study aims at testing the effect of using a proactive approach entailing periodic email prompts compared to a reactive approach on revisits to an online CT lifestyle program. When periodic prompts are proven to be effective in stimulating sustained use and, possibly, behaviour change and improved health, this could lead to specific recommendations on the use of prompts in online lifestyle interventions. Also, with regard to the use of periodic prompting, results from this study may be further refined by investigating not only 
the optimal frequency and methods by which respondents should be prompted, but also the content of the prompts. 


\section{Chapter 3}

The use of a proactive dissemination strategy to optimize reach of an Internet-delivered computer tailored lifestyle intervention 


\section{ABSTRACT}

The use of reactive strategies to disseminate effective Internet-delivered lifestyle interventions restricts their level of reach within the target population. This stresses the need to invest in proactive strategies to offer these interventions to the target population. The present study used a proactive strategy to increase reach of an Internet-delivered multi-component computer tailored intervention, by embedding the intervention in an existing online health monitoring system of the Regional Public Health Services in the Netherlands. The research population consisted of Dutch adults who were invited to participate in the Adult Health Monitor ( $N=96,388$ ) offered by the Regional Public Health Services. This Monitor consisted of an online or a written questionnaire. A prospective design was used to determine levels of reach, by focusing on actual participation in the lifestyle intervention. Furthermore, adequacy of reach among the target group was assessed by composing detailed profiles of intervention users. Participants' characteristics, like demographics, behavioural and mental health status and quality of life, were included in the model as predictors. A total of 41,155 (43\%) people participated in the Adult Health Monitor, of which $41 \%(n=16,940)$ filled out the online version. More than half of the online participants indicated their interest $(n=9169 ; 54 \%)$ in the computer tailored intervention and 5168 participants (31\%) actually participated in the Internet-delivered computer tailored intervention. Males, older respondents and individuals with a higher educational degree were significantly more likely to participate in the intervention. Furthermore, results indicated that especially participants with a relatively healthier lifestyle and a healthy BMI were likely to participate. With one out of three online Adult Health Monitor participants actually participating in the computer tailored lifestyle intervention, the employed proactive dissemination strategy succeeded in ensuring relatively high levels of reach. Reach among at-risk individuals (e.g. low socioeconomic status and unhealthy lifestyle) was modest. It is therefore essential to further optimize reach by putting additional effort into increasing interest in the lifestyle intervention among at-risk individuals and to encourage them to actually use the intervention. 


\section{INTRODUCTION}

Lifestyle behaviours, such as smoking, unhealthy eating habits, physical inactivity and excessive alcohol consumption are established risk factors for chronic diseases, most importantly for cardiovascular diseases, different types of cancer and diabetes type II (RIVM, 2012; World Health Organisation, 2011). The high prevalence of these behaviours (RIVM, 2012) and their tendency to co-occur (De Vries, et al., 2008; Strecher et al., 2002), stresses the need to invest in effective lifestyle interventions. Currently, lifestyle interventions are more and more frequently disseminated through the Internet. Due to the high level of reach and accessibility of the Internet (Internet World Stats, 2013), it offers a good platform for the dissemination of tailored and targeted interventions to the general public (Brug et al., 2005; Internet World Stats, 2013; Lustria et al., 2009; Ritterband et al., 2003).

Especially interventions using computer tailored techniques have reported positive effects (Krebs et al., 2010; Lustria et al., 2009; Ritterband et al., 2003), e.g. in the fields of physical activity (Van den Berg et al., 2007; Vandelanotte et al., 2007), fruit and vegetable intake (Brug et al., 1998; Neville et al., 2009), smoking (Borland et al., 2004; Dijkstra, De Vries, \& Roijackers, 1998) and alcohol consumption (Riper et al., 2008; White et al., 2010). This potentially high level of reach, combined with the proven effectiveness of computer tailored interventions, would suggest that interventions offered through the Internet hold the promise to significantly contribute to an increased impact on public health (Glasgow et al., 1999).

However, despite the promising prospects of the Internet, actual reach of effective interventions within the target population remains inadequate (Evers et al., 2005; Eysenbach, 2005; Glasgow et al., 2007; Portnoy et al., 2008), as only a limited proportion of people is actually reached by the interventions (Brouwer et al., 2009; Evers et al., 2005). These suboptimal levels of reach might be partially explained by the fact that most effective interventions are offered reactively to the target population 
(Prochaska et al., 2001). The use of reactive dissemination strategies implies that a rather passive approach is taken in which users themselves must undertake action in order to use and optimally benefit from the intervention content (Prochaska et al., 1997). Potential users must actively look and register for existing lifestyle initiatives. However, several barriers prevent large proportions of the population from actively seeking assistance in changing their lifestyle behaviours. The barriers pertain to insufficient knowledge on the existence of interventions or places where they can be found, unawareness of the current status of their lifestyle behaviours or a lack of motivation to change their lifestyle (Ajzen, 1991; De Vries et al., 2003). This stresses the necessity to invest in strategies through which lifestyle interventions can be proactively offered to the target population. These strategies have the potential to diminish important barriers for use and increase the knowledge of existing, evidence-based interventions (Schneider, van Osch, Schulz, Kremers, \& de Vries, 2012; Velicer et al., 1999).

Within the present study, a proactive strategy was used to increase reach of an Internet-delivered multi component computer tailored intervention, focusing on physical activity, fruit and vegetable consumption, alcohol intake and smoking behaviour. In order to proactively offer the intervention to the target population, the intervention was embedded in a nationwide survey, the Adult Health Monitor, of two of the Regional Public Health Services (RPHS) in the Netherlands (GGD Nederland, 2011). As part of their statutory obligation to periodically monitor the health of the Dutch adult population, this survey is used to assess overall levels of health, as well as different aspects of general health, e.g. physical, mental and social health among a representative sample with an interval of four years. Based on findings of this monitoring tool, future health policies at a national, provincial and local level are outlined. Integrating the Internet-delivered computer tailored intervention in the Adult Health Monitor was expected to have several benefits. Most importantly, the Adult Health Monitor was expected to provide an important access point for reaching a considerably large segment of the Dutch population with an evidence-based intervention. Furthermore, by integrating the computer tailored intervention in the Adult Health Monitor, awareness of the existence of the intervention could be increased. Thirdly, by embedding the intervention in the Adult Health Monitor, data can directly be obtained and presented in order to inform people on the status of their current lifestyle. This may increase the level of lifestyle awareness and serve as an important cue to action to positively change their current lifestyle. Finally, integrating an Internet-delivered computer tailored intervention will enable the RPHS's to optimize their public health education task; besides solely monitoring health behaviour, the RPHS's now have an opportunity to provide people with personalized advice on how to effectively change their lifestyle 
status. The main aim of this study was to determine the effect of this proactive dissemination strategy on reach of the Internet-delivered computer tailored intervention. Within the RE-AIM framework, reach is defined as the percentage of people invited and eligible for participation that actually participate in the intervention (Glasgow et al., 1999). Therefore, success of this proactive dissemination strategy was assessed by studying actual levels of participation in the computer tailored lifestyle intervention among people included in the Adult Health Monitor sample. Furthermore, the second aim of this study was to determine adequacy of reach among the target population (Velicer et al., 1999) by composing and analyzing detailed profiles of computer tailored intervention users (Glasgow et al., 1999; Rogers, 1995).

\section{METHODS}

Within the current study the level of reach of an Internet-delivered computer tailored lifestyle intervention offered through the Adult Health Monitor was examined. Furthermore, adequacy of reach among the target group was assessed by composing detailed profiles of computer tailored intervention users. This study was approved by the Medical Ethics Committee of Maastricht University and the University Hospital Maastricht (NL27235.068.09/MEC 09-3-016) (Schneider et al., 2011; Schulz et al., 2011). Data for the present study were collected from October 2009 until July 2010.

\section{Participants and inclusion criteria}

The research population consisted of adults (19-64 years) living in the Dutch Provinces of Zeeland and North-Brabant who participated in the Adult Health Monitor 2009. Additional inclusion criteria for participation in the computer tailored intervention were that participants had to be able to understand the Dutch language sufficiently, had to have access to the Internet and had to have a valid email address. There were no explicit exclusion criteria stated for the current study. Participants had to consent to their participation by filling out an online informed consent form.

\section{Recruitment and procedure}

In the fall of 2009, four RPHS's of the provinces North-Brabant and Zeeland invited a representative sample of inhabitants of these provinces to participate in the Monitor 2009. A total of 96,388 participants received an invitation to participate by postal mail. This invitation included information on the content and purpose of the Adult Health Monitor. Furthermore, a return envelope was included to return the questionnaire after completion. Besides filling out the written Adult Health Monitor questionnaire, participants were also offered an opportunity to fill out an online version of the questionnaire. For this purpose, a link to the website was included in the invitation, as 
well as personal log-in information. Since the Internet-delivered computer tailored lifestyle intervention was a web-based intervention, it was only available for participants that decided to fill out the online version of the monitor questionnaire. Online participation was therefore encouraged by informing people about the embedded intervention in the invitation letter and by pointing out its exclusive availability for online participants.

After completing the Adult Health Monitor, all participants of the online version were introduced to the embedded computer tailored lifestyle intervention. Participants were explained that the intervention was free of charge and provided an opportunity to receive personalized computer tailored feedback about their health behaviours. Participants indicating to be interested in the new intervention were asked to leave their email address and to fill out an online form indicating their consent to participate in the current study. Approximately three weeks after completion of the Adult Health Monitor, all interested participants received an email invitation to log in to the computer tailored intervention with a personal login code and password. After approximately one month, non-responders received an additional email reminding them about their interest in the intervention and inviting them to participate. After logging on to the intervention, participants received detailed information on its goal and content. Data on demographic and behavioural characteristics obtained through the Adult Health Monitor were transported to the computer tailored intervention.

\section{Materials}

The computer tailored intervention integrates interventions that have been tested and proven to be effective in randomized control trials for increasing smoking cessation, promoting the intake of fruit and vegetables, increasing the level of physical activity and reducing the consumption of alcohol (Brug et al., 1999; Poel et al., 2009; Smeets et al., 2008; Van Keulen et al., 2011). The intervention used a dual approach to guide people towards behaviour change. The first part consisted of a health risk appraisal and compared participants' health behaviour status to the Dutch public health guidelines set for these behaviours (i.e. being moderately physically active for 30 minutes at least five days a week (Hildebrandt, Chorus, \& Stubbe, 2010), eating two pieces of fruit per day and eating 200 grams of vegetables per day (Gezondheidsraad, 2006), not drinking more than one (women) or two (men) glasses of alcohol a day (Alcoholinfo.nl, 2012) and non-smoking (Kwaliteitsinstituut voor de Gezondheidszorg CBO, 2004)). We used feedback messages to inform people on the current status of each health behaviour. All messages were complemented by using graphic representations of traffic lights (Strecher et al., 2008), with a green light corresponding to adherence to the guidelines and a red light corresponding to non-adherence. An orange 
light was used for people that were close to adherence to these guidelines. In case of discrepancies between current behaviour and the guidelines, people were alerted and directed to the computer tailored modules (Schulz et al., 2011).

Second, participants were provided with assistance in changing their health behaviour by means of five separate computer tailored modules on physical activity, fruit and vegetable intake, alcohol consumption and smoking cessation. The content of these modules was based on the Integrated Model for exploring motivational and behavioural change (I-Change Model) (De Vries et al., 2003) and used a fixed, gradual approach to guide people towards behaviour change. Within the modules, all health advices were adapted to individuals' demographic, behavioural and cognitive characteristics (De Vries \& Brug, 1999; Kreuter et al., 2000). Demographic and behavioural characteristics, like participants' gender and current health behaviour status were directly obtained through the Adult Health Monitor. Cognitive variables, such as attitude, perceived social influence, self-efficacy, intention and action planning strategies (preparatory plans and coping plans) were assessed by using an additional tailoring questionnaire. An extensive description of the intervention content is described elsewhere (Schneider et al., 2011; Schulz et al., 2011).

\section{Measures}

User characteristics were collected in order to produce detailed profiles of people who decided to participate in the computer tailored intervention in order to determine adequacy of reach. These profiles entailed information on demographic characteristics, health behaviour status, mental health status and quality of life, obtained through the Adult Health Monitor.

\section{Demographic characteristics}

Demographic characteristics included questions on age, gender (1=male; 2=female), height, weight, marital status (widower, divorced, unmarried (not in a relationship $=1$ ); or living together, married (in a relationship=2)), native country (1=not from the Netherlands; 2 =from the Netherlands), educational level (no education, primary or lower vocational school (low=1); secondary vocational school or high school (medium=2); or higher professional education or university (high=3)) and current work status (unemployed=1; employed=2). Data on participants' height (in meters) and weight (in kilograms) were used to compose a Body Mass Index (BMI). This index was defined by dividing participants' body weight $(\mathrm{kg})$ by the square of participants' height (m) and was categorized as $<18.5=1$ (underweight); $\geq 18.5$ to $<25=2$ (normal weight); $\geq 25$ to $<30=3$ (overweight); $\geq 30=4$ (obesity). 
Health behaviour status

Health behaviour status was objectively assessed by measuring level of physical activity, fruit and vegetable intake, alcohol consumption and smoking behaviour. Physical activity was measured by the Short QUestionnaire to ASsess Health enhancing physical activity (SQUASH) (Wendel Vos et al., 2003). Fruit and vegetable intake were assessed using the Food-Frequency-Questionnaire (FFQ) for fruit and vegetable intake (Bogers et al., 2004; Van Assema et al., 2002). Alcohol consumption was measured by the Dutch Quantity-Frequency-Variability Questionnaire (QFV) (Lemmens et al., 1992) and smoking status was assessed by asking participants whether they smoked, what they smoked (cigarettes, cigars, packets pipe tobacco) and how much they smoked per day (cigarettes) and per week (cigars/ packets pipe tobacco). Furthermore, an additional item was used to obtain a more subjective assessment of participants' lifestyle status. This item assessed the degree to which participants considered their own current lifestyle to be healthy and was measured on a five point scale (1=very unhealthy; $5=$ very healthy).

\section{Mental health status}

Mental health status was assessed by using the Kessler Psychological Distress Scale (K10) (Andrews \& Slade, 2001; Kessler et al., 2002). This scale assesses the occurrence of non-specific psychological distress using ten items measured on a five point scale ( $1=$ none of the time; $5=$ all of the time). A sum score was computed, with higher scores indicating higher levels of distress.

\section{Quality of life}

Quality of life was assessed using the Medical Outcomes Study 12-Item Short Form Health Survey (SF-12) (Jenkinson et al., 1997; Ware, Kosinski, \& Keller, 1996).

\section{Outcome measures}

The primary outcome measure was level of actual participation in the intervention. Secondary outcome measures were medium of participation and level of interest in the intervention. Dichotomous variables were created in order to examine level of participation ( $0=$ no participation/ $1=$ participation), medium of participation ( $0=$ written questionnaire/1=online questionnaire) and level of interest in the intervention $(0=$ not interested/1=interested). Participants of the online Adult Health Monitor were asked to indicate their interest in the intervention by leaving their email address. Interest in the intervention was labelled as "yes" when people filled out their email address. Furthermore, participation was objectively monitored by looking at log-in data and was labelled as "yes" when people responded to the email invitation by logging in to 
the computer tailored intervention.

\section{Statistical analyses}

First, general descriptive statistics were used to describe user characteristics of the participants, by focusing on demographic characteristics, health behaviour status, mental health status and quality of life as well as level of interest and participation in the computer tailored intervention. Second, logistic regression analyses were conducted. Medium of participation, interest and participation in the computer tailored intervention were the dependent variables. Demographic characteristics (age, gender, $\mathrm{BMI}$, marital status, native country, educational level and current work status), health behaviour status, mental health status and quality of life were included in the model as predictors. An alpha of .05 was used to indicate statistical significance. All statistical analyses were done with the program SPSS 19.0.

\section{RESULTS}

\section{Reach of the proactive dissemination strategy}

In total, 41.155 (43\%) people participated in the Adult Health Monitor 2009 in the provinces of North-Brabant and Zeeland; 24.215 participants (59\%) filled out the written version and 16.940 participants (41\%) filled out the online version (Figure 3.1). All online participants were introduced to the Internet-delivered computer tailored lifestyle intervention and 9.169 participants (54\%) actually indicated to be interested in the intervention. Finally, all participants that indicated their interest were invited to use the intervention. This resulted in a total of 5.168 participants ( $31 \%$ of the online sample and $56 \%$ of the sample of people interested in participating) logging in to the Internet-delivered computer tailored intervention.

\section{Baseline characteristics of intervention users}

Users of the Internet-delivered computer tailored intervention had a mean age of 44 years $(S D=12.67)$, around $54 \%$ was male and almost $50 \%$ had a medium educational level (Table 3.1). Furthermore, a little over $75 \%$ of all participants was employed and in a relationship and $95 \%$ was born in the Netherlands. Approximately $50 \%$ of all participants had a healthy body weight, whereas a little less than $2 \%$ of all visitors was underweight, $35 \%$ was overweight and $10 \%$ was obese. With regard to physical activity, $21 \%$ did not comply with the Dutch guidelines, whereas $46 \%$ and $69 \%$ were not adhering to the Dutch guidelines of fruit and vegetables intake respectively. One out of five visitors (19\%) indicated that they smoked, whereas approximately one of four participants (28\%) did not comply with the Dutch guidelines for alcohol intake. In total, the largest group of people complied with three lifestyle guidelines (36\%), 
whereas a very limited percentage of people complied with none of these guidelines (1\%) or all of the guidelines (10\%). Characteristics of all Adult Health Monitor participants, online Adult Health Monitor participants and people that indicated their interest in the Internet-delivered computer tailored lifestyle intervention are also listed in Table 3.1.

\section{Figure 3.1. Flowchart of Adult Health Monitor and CT lifestyle intervention participation}

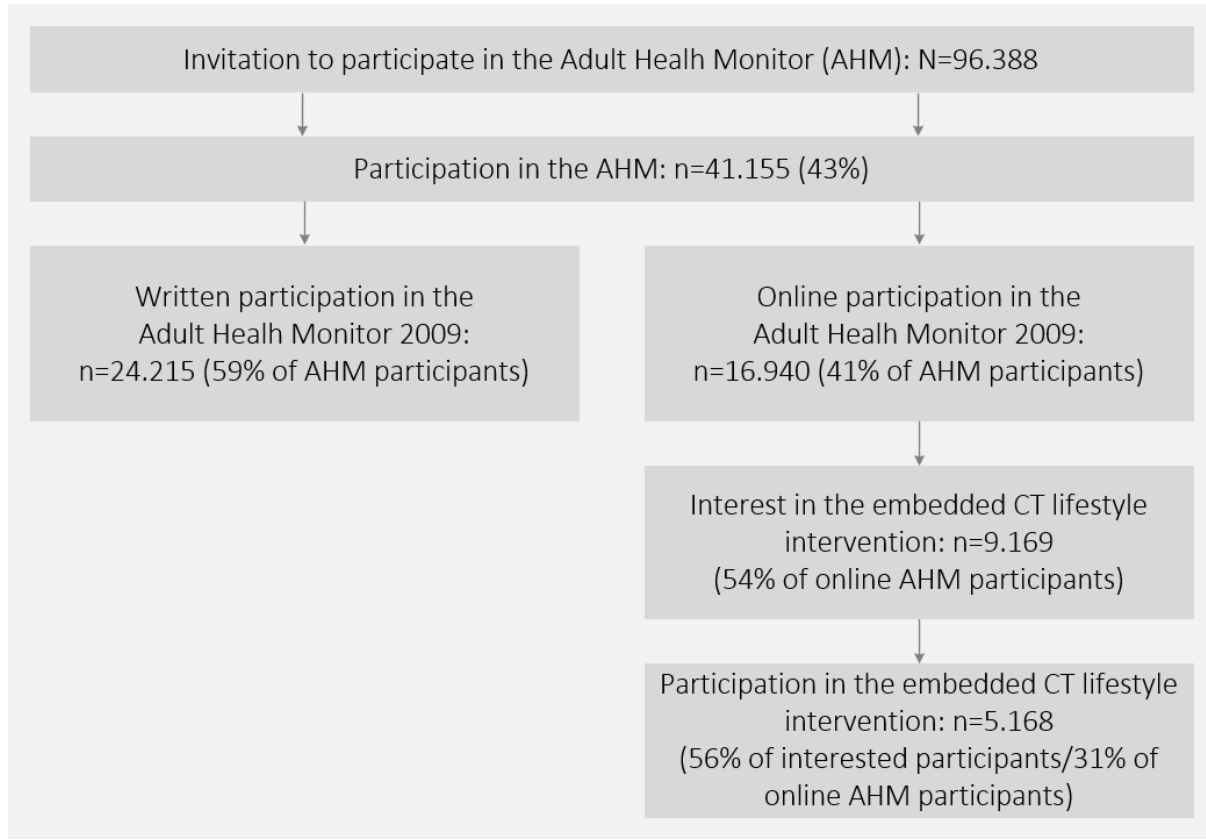

\section{Adequacy of reach}

Participation in the Adult Health Monitor

Results of multiple logistic regression analyses indicated that younger $(\mathrm{OR}=0.99 ; \mathrm{Cl}$ 0.99 - 0.99; $\mathrm{p}<0.05)$, male $(\mathrm{OR}=0.65 ; \mathrm{Cl} 0.62-0.68 ; \mathrm{p}<0.05)$ and relatively highly educated participants $(\mathrm{OR}=2.63 ; \mathrm{Cl} 2.45-2.81 ; \mathrm{p}<0.05)$, as well as participants that were employed $(\mathrm{OR}=1.06 ; \mathrm{Cl} 1.00-1.12 ; \mathrm{p}<0.05)$ and in a relationship $(\mathrm{OR}=1.09$; $\mathrm{Cl} 1.03-1.15 ; \mathrm{p}<0.05)$ were more inclined to fill out the online version of the Adult Health Monitor. Furthermore, participants with an unhealthier lifestyle (fewer guidelines complied with) were more likely to fill out the online version of the Adult Health Monitor (OR = 0.97; $\mathrm{Cl} 0.95-0.99 ; \mathrm{p}<0.05)$. 
Table 3.1. Characteristics of Adult Health Monitor participants ( $N=41.155$ )

\begin{tabular}{|c|c|c|c|c|}
\hline Characteristics & $\begin{array}{c}\text { Total } \\
\text { sample } \\
\mathrm{N}=41.155\end{array}$ & $\begin{array}{c}\text { Online } \\
\text { completion } \\
n=16.940\end{array}$ & $\begin{array}{l}\text { Interest } \\
n=9.169\end{array}$ & $\begin{array}{l}\text { Participation } \\
\text { n=5.168 }\end{array}$ \\
\hline Age (19-64) (Mean, SD) & $44.78,12.70$ & $43.19,12.42$ & $43.23,12.77$ & $43.94,12.67$ \\
\hline \multicolumn{5}{|l|}{ Gender } \\
\hline Male & 44.7 & 51.1 & 52.6 & 53.8 \\
\hline Female & 55.3 & 48.9 & 47.4 & 46.2 \\
\hline \multicolumn{5}{|l|}{ Education level (\%) } \\
\hline Low & 23.2 & 14.7 & 13.2 & 10.6 \\
\hline Medium & 48.5 & 48.9 & 47.7 & 46.9 \\
\hline High & 28.2 & 36.4 & 39.1 & 42.6 \\
\hline \multicolumn{5}{|l|}{ Work situation (\%) } \\
\hline Employed & 72.8 & 77.1 & 76.5 & 76.3 \\
\hline Unemployed & 27.2 & 22.9 & 23.5 & 23.7 \\
\hline \multicolumn{5}{|l|}{ Marital status (\%) } \\
\hline In a relationship & 76.8 & 77.0 & 75.4 & 76.0 \\
\hline Not in a relationship & 24.2 & 23.0 & 24.6 & 24.0 \\
\hline \multicolumn{5}{|l|}{ Native country (\%) } \\
\hline The Netherlands & 93.3 & 93.5 & 93.8 & 95.0 \\
\hline Other & 6.7 & 6.5 & 6.2 & 5.0 \\
\hline \multicolumn{5}{|l|}{ BMI $\left(\mathrm{kg} \mathrm{m}^{-2}\right)(\%)$} \\
\hline$<18.5$ & 1.5 & 1.4 & 1.5 & 1.7 \\
\hline$\geq 18.5$ to 25 & 50.9 & 51.5 & 51.1 & 52.6 \\
\hline$\geq 25$ to $<30$ & 35.1 & 35.4 & 35.6 & 35.4 \\
\hline$\geq 30$ & 12.4 & 11.7 & 11.8 & 10.4 \\
\hline \multicolumn{5}{|l|}{ Physical activity (\%) } \\
\hline Adherence & 78.4 & 77.1 & 77.7 & 79.1 \\
\hline Non-adherence & 21.6 & 22.9 & 22.3 & 20.9 \\
\hline \multicolumn{5}{|c|}{ Vegetable consumption (\%) } \\
\hline Adherence & 31.2 & 29.9 & 30.4 & 31.5 \\
\hline Non-adherence & 68.8 & 70.1 & 69.6 & 68.5 \\
\hline \multicolumn{5}{|l|}{ Fruit consumption (\%) } \\
\hline Adherence & 49.7 & 46.1 & 45.8 & 45.9 \\
\hline Non-adherence & 50.3 & 53.9 & 54.2 & 54.1 \\
\hline \multicolumn{5}{|l|}{ Smoking behavior (\%) } \\
\hline Adherence & 74.7 & 77.6 & 78.1 & 81.7 \\
\hline Non-adherence & 25.3 & 22.4 & 21.9 & 18.3 \\
\hline \multicolumn{5}{|l|}{ Alcohol intake (\%) } \\
\hline Adherence & 73.6 & 73.6 & 72.3 & 71.7 \\
\hline Non-adherence & 26.4 & 26.4 & 27.7 & 28.3 \\
\hline \multicolumn{5}{|l|}{ Total \# of guidelines } \\
\hline 0 & 0.9 & 0.9 & 0.9 & 0.7 \\
\hline 1 & 7.2 & 7.5 & 7.3 & 6.5 \\
\hline 2 & 21.3 & 22.1 & 22.4 & 20.9 \\
\hline 3 & 33.6 & 34.1 & 33.9 & 35.5 \\
\hline 4 & 27.1 & 26.2 & 26.3 & 26.6 \\
\hline 5 & 9.9 & 9.1 & 9.2 & 9.7 \\
\hline \multicolumn{5}{|l|}{ Personal judgment lifestyle } \\
\hline Very unhealthy & 5.7 & 5.5 & 5.6 & 6.5 \\
\hline Unhealthy & 61.8 & 61.6 & 60.3 & 61.8 \\
\hline
\end{tabular}


Table 3.1. continued: Characteristics of Adult Health Monitor participants ( $N=41.155$ )

\begin{tabular}{lcccc}
\hline Characteristics & $\begin{array}{c}\text { Total } \\
\text { sample } \\
\mathrm{N}=41.155\end{array}$ & $\begin{array}{c}\text { Online } \\
\text { completion } \\
\mathrm{n}=16.940\end{array}$ & $\mathrm{n}=9.169$ & $\mathrm{n}=5.168$ \\
\cline { 2 - 5 } Not unhealthy/not healthy & 28.9 & 28.9 & 29.4 & 27.5 \\
Healthy & 3.2 & 3.7 & 4.3 & 3.9 \\
Very healthy & 0.4 & 0.3 & 0.4 & 0.3 \\
K10 (Mean, SD) & $15.41,6.26$ & $15.17,5.90$ & $15.47,6.08$ & $15.21,5.71$ \\
SF-12 (Mean, SD) & $39.76,5.63$ & $40.09,5.29$ & $39.88,5.39$ & $40.12,5.19$ \\
\hline
\end{tabular}

Interest in the Internet-delivered lifestyle intervention

Results of multiple logistic regression analyses indicated that younger $(\mathrm{OR}=0.99 ; \mathrm{Cl}$ $0.99-0.99 ; p<0.05)$, male $(O R=0.65 ; \mathrm{Cl} 0.62-0.68 ; p<0.05)$ and relatively highly educated participants $(\mathrm{OR}=2.63 ; \mathrm{Cl} 2.45-2.81 ; \mathrm{p}<0.05)$, as well as participants that were employed $(\mathrm{OR}=1.06 ; \mathrm{Cl} 1.00-1.12 ; \mathrm{p}<0.05)$ and in a relationship $(\mathrm{OR}=1.09$; $\mathrm{Cl} 1.03-1.15 ; \mathrm{p}<0.05)$ were more inclined to fill out the online version of the Adult Health Monitor. Furthermore, participants with an unhealthier lifestyle (fewer guidelines complied with) were more likely to fill out the online version of the Adult Health Monitor (OR = 0.97; Cl $0.95-0.99 ; \mathrm{p}<0.05)$.

Table 3.2. Predictors of participation in the $\mathrm{CT}$ lifestyle intervention ( $\mathrm{n}=9.169)$

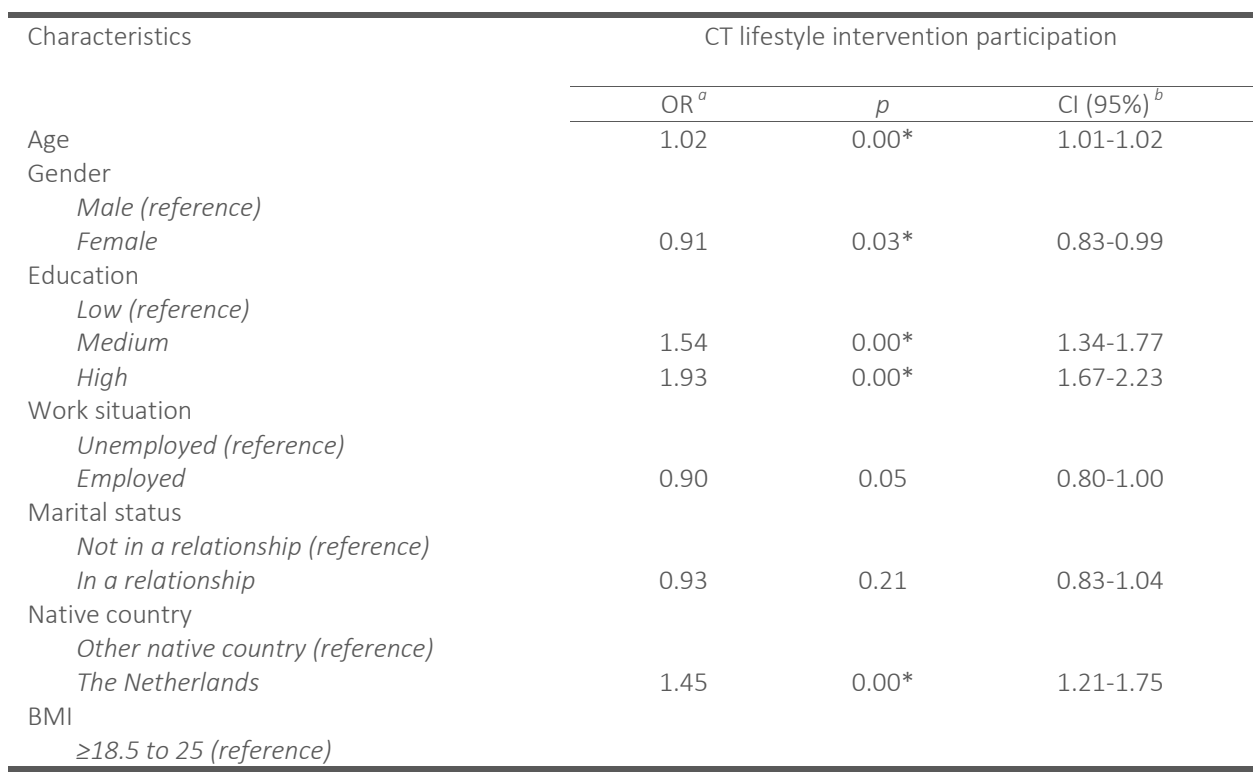


Table 3.2. continued: Predictors of participation in the $\mathrm{CT}$ lifestyle intervention ( $\mathrm{n}=9.169)$

Characteristics

$<18.5$

$\geq 25$ to $<30$

$\geq 30$

Total \# of guideline (adherence)

Personal judgment own current lifestyle

K10

SF-12

CT lifestyle intervention participation

\begin{tabular}{ccc}
\hline $\mathrm{OR}^{a}$ & $p$ & $\mathrm{Cl}(95 \%)^{b}$ \\
\hline 1.55 & $0.02^{*}$ & $1.06-2.27$ \\
0.88 & $0.01^{*}$ & $0.79-0.97$ \\
0.71 & $0.00^{*}$ & $0.61-0.82$ \\
1.06 & $0.01^{*}$ & $1.01-1.10$ \\
1.06 & 0.14 & $0.98-1.15$ \\
1.00 & 0.82 & $0.99-1.01$ \\
1.00 & 0.56 & $0.99-1.02$
\end{tabular}

${ }^{a}$ Odds ratio; ${ }^{b}$ Confidence interval.

$* p<.05$

Use of the Internet-delivered lifestyle intervention

Results of multiple logistic regression analyses indicated that male $(\mathrm{OR}=0.91 ; \mathrm{Cl} 0.83$ - 0.99; $\mathrm{p}<0.05)$, older $(\mathrm{OR}=1.02 ; \mathrm{Cl} 1.01-1.02 ; \mathrm{p}<0.05)$ and relatively highly educated participants $(\mathrm{OR}=1.93 ; \mathrm{Cl} 1.67-2.23 ; \mathrm{p}<0.05)$ were more likely to participate in the intervention by logging in to the intervention (see Table 3.2). Furthermore, intervention participants were more likely to be from the Netherlands $(O R=1.45 ; \mathrm{Cl}$ $1.21-1.75 ; p<0.05)$. Finally, participants with a relatively healthier lifestyle (more guidelines complied with) $(\mathrm{OR}=1.06 ; \mathrm{Cl} 1.01-1.10 ; \mathrm{p}<0.05)$ and underweighted participants $(\mathrm{OR}=1.55 ; \mathrm{Cl} 1.06-2.27 ; \mathrm{p}<0.05)$ were more likely to participate in the computer tailored intervention, whereas overweighed $(\mathrm{OR}=0.88 ; \mathrm{Cl} 0.79-0.97 ; \mathrm{p}<$ $0.05)$ and obese participants $(O R=0.71 ; \mathrm{Cl} 0.61-0.82 ; \mathrm{p}<0.05)$ were less likely to participate.

\section{DISCUSSION}

Within the current study, a proactive dissemination strategy was used to optimize reach of an Internet-delivered computer tailored lifestyle intervention. This proactive strategy consisted of embedding the intervention in the Adult Health Monitor of the RPHS's of two Dutch provinces. Success of this strategy was determined by studying actual levels of participation in the computer tailored lifestyle intervention. Furthermore, we investigated adequacy of reach by studying user characteristics that predicted interest en participation in the computer tailored lifestyle intervention.

\section{Reach of the proactive dissemination strategy}

More than half of the online Adult Health Monitor participants indicated their interest in the intervention. Moreover, more than half of all interested participants actually participated in the computer tailored lifestyle intervention, which is one out of three 
invited and eligible participants. It is difficult to compare these results to other studies that study reach in a real world setting, since actual estimates of reach are scarce (Bennett \& Glasgow, 2009). However, a multi-risk lifestyle behaviour intervention offered to a comparable sample reported levels of reach around $3 \%$ of the eligible participants (Brouwer et al., 2010). Results from efficacy trials have indicated that actual reach of the intervention is suboptimal (Brouwer et al., 2010; Glasgow et al., 2007; Leslie et al., 2005), with participation rates ranging from $2.5 \%$ to $20 \%$. Considering these results, the current proactive dissemination strategy can be regarded as successful.

\section{Adequacy of reach}

The current study also aimed at examining adequacy of reach among the target group (i.e. at-risk individuals). This was done by composing profiles of users and by examining which user characteristics predicted both interest and participation in the intervention. Results indicated that especially older, male and relatively highly educated participants were inclined to indicate their interest in the intervention. Furthermore, participants that were unemployed, not in a relationship and born in the Netherlands were more likely to be interested in the computer tailored intervention. Finally, participants with relatively more symptoms of depression and anxiety were more likely to be interested in the computer tailored intervention. Even though the effect of employment status, marital status and depressive symptomatology diminished with regard to actual participation in the intervention, the effect of education and native country remained, with relatively higher educated participants and participants from the Netherlands being more willing to participate in the intervention. This finding is in line with previous studies examining use of Internet-delivered lifestyle interventions, where the majority of participants had a relatively high educational level (Brouwer et al., 2010; Lorence \& Park, 2008; Spittaels, De Bourdeaudhuij, Brug, et al., 2007; Yang et al., 2008). The level of reach was modest in other subgroups, as results indicated that especially participants with a relatively healthier lifestyle and a healthy BMI were likely to actually participate in the intervention. These results are to a large extent in line with previous findings that Internet-delivered computer tailored interventions tend to be predominantly used by people with a healthy lifestyle (Brouwer et al., 2010; Spittaels, De Bourdeaudhuij, Brug, et al., 2007; Strecher et al., 2008; Verheijden et al., 2007).

\section{Participation in the Adult Health Monitor}

Of the sample that was selected to participate in the Adult Health Monitor 2009, a little over forty per cent accepted the invitation to participate. Even though the over- 
all percentages of people that participated in the Monitor has decreased compared to the Adult Health Monitor conducted in 2005 (GGD Nederland, 2012), the percentage of people that decided to participate using the online monitoring questionnaire has substantially increased. In 2005, only one out of four respondents preferred to use the online questionnaire (GGD Nederland, 2012). This increase is in line with the rapidly expanding penetration rates of the Internet in the Netherlands. Since 2006, Internet access rates have increased from 65\% to almost 93\% of all Dutch inhabitants in 2013 (Internet World Stats, 2013), allowing for an increased amount of people filling out the online AHM questionnaire.

Participation in the online version of the Adult Health Monitor was preferred by people with an unhealthier lifestyle compared to the written version. These results imply that using the online Adult Health Monitor is a good tool to reach those people that are expected to benefit most from lifestyle interventions (i.e. people that engage in health risk behaviours). However, results on actual participation in the intervention imply that the proactive strategy did not succeed in persuading these at-risk people to actually participate in the embedded computer tailored lifestyle intervention. Even though suboptimal participation rates among people with an unhealthy lifestyle are common for most computer tailored lifestyle interventions (Brouwer et al., 2010; Spittaels, De Bourdeaudhuij, Brug, et al., 2007; Strecher et al., 2008; Verheijden et al., 2007), the online environment of the Adult Health Monitor offers good opportunities to reach at-risk groups. However, these opportunities might be further optimized in the future. Direct transportation of data from the Adult Health Monitor to the computer tailored lifestyle intervention allowed for composition of an individual health overview addressing all relevant lifestyle behaviors. However, this overview was only presented to participants that were interested in the intervention and decided to actually participate. Impact of this proactive strategy might be further improved if this overview could be presented to all Adult Health Monitor participants, instead of only interested participants. The overview provided personalized information on the status of individuals' current lifestyle behaviours, as well as additional information regarding the guidelines set for these behaviours. As a result, the detailed content of this overview was very suitable to increase awareness of people's current lifestyle status and to point out discrepancies with the guidelines set for these behaviours. This overview may then serve as an important cue to action to change their lifestyle among people with an unhealthy lifestyle.

Considering the promising results of the used proactive dissemination strategy with regard to actual levels of reach, the Adult Health Monitor can be regarded as a suitable and promising vehicle to disseminate evidence-based Internet-delivered lifestyle interventions to the general public. The proportion of people reached shows 
the potential for broad reach when implemented on a national level. Offering effective interventions through the Adult Health Monitor environment at a national level can therefore serve as an important access point to reach the target population. As a consequence, public health impact of these interventions can be further optimized. Even though we partly succeeded in reaching the target population of at-risk individuals, adequacy of reach may be further optimized by putting additional effort into the recruitment of online and at-risk participants. It is therefore recommendable to explore which promotion strategies should be used to attract especially older and lower educated people to the online Adult Health Monitor questionnaire. Furthermore, effort should be put into increasing interest in the computer tailored lifestyle intervention among online Adult Health Monitor participants and to persuade them to actually use the intervention.

\section{Strengths and limitations}

One of the major strengths of this study was the opportunity to proactively offer an Internet-delivered computer tailored lifestyle intervention to a large sample of Dutch inhabitants within the controlled environment of the Adult Health Monitor. This allowed us to carefully determine the number of people invited and eligible for participation, as well the number of actual intervention participants. As a result, valuable information was obtained regarding actual numbers of reach of the used dissemination strategy. Furthermore, detailed information was gathered regarding not only characteristics of people that were interested and participated in the intervention, but also of people that refrained from participation. This is exceptional, since a problem encountered in most studies evaluating dissemination strategies is the accuracy by which all non-users can be tracked. Thus, the current study provided a unique possibility to obtain information on so-called non-users (e.g. people not interested in the intervention or people that were interested, but refrained from actual participation).

The study also suffered from several limitations. First, the sample used for this study was a convenience sample, consisting of people from two Dutch provinces. However, it is important to stress that all RPHS's invite an initial sample which is representative of the Dutch population, to participate in the Adult Health Monitor (GGD Nederland, 2011). However, this study might have been susceptible to selection bias. Within this study, we tried to optimize reach of a computer tailored lifestyle intervention by embedding the intervention in the Adult Health Monitor. Since this intervention was an Internet-delivered intervention, it was only offered to people that decided to fill out the electronic version of the Adult Health Monitor. Results have indicated that these specific electronic participants tend to be younger, have a relatively higher education and an unhealthier lifestyle. As a result, data on actual intervention partici- 
pation were obtained from a selective sample. This sample does not provide a good cross-section of the general Dutch population (CBS, 2012), which implies that the obtained results should be generalized with caution. Previous studies have, however, indicated that Internet-delivered computer tailored interventions tend to predominantly reach higher educated people (Brouwer, Kroeze, et al., 2011; Schneider, van Osch, Schulz, et al., 2012), which might imply that our sample partly corresponds to the subgroup of people known to be reached by Internet-delivered computer tailored interventions. Although it does not represent the general population, the obtained results are still valuable in the context of Internet-delivered computer tailored lifestyle interventions.

\section{Conclusions}

The present study used a proactive strategy to increase reach of an Internet-delivered multi component computer tailored intervention, by embedding the intervention in an existing online health monitoring system of the Regional Public Health Services in the Netherlands. With one out of three online Adult Health Monitor participants actually using the intervention, the employed proactive dissemination strategy succeeded in ensuring relatively high levels of reach. With regard to adequacy of reach result from this study indicated that reach among at-risk individuals (e.g. low socioeconomic status and unhealthy lifestyle) remained modest. We therefore recommend to further optimize reach by putting additional effort into increasing interest in the lifestyle intervention among at-risk individuals and to encourage them to actually use the intervention. 


\title{
Chapter 4
}

\author{
Identifying factors for optimal \\ development of health-related \\ websites: a Delphi study among \\ experts and potential future users
}




\section{ABSTRACT}

The Internet has become a popular medium for offering tailored and targeted health promotion programs to the general public. However, suboptimal levels of program use in the target population limit the public health impact of these programs. Optimizing program development is considered as one of the main processes to increase usage rates. The objective of this study was to distinguish factors potentially related to optimal development of health-related websites by involving both experts and potential users. By considering and incorporating the opinions of experts and potential users in the development process, involvement in the program is expected to increase, consequently resulting in increased appreciation, lower levels of attrition, and higher levels of sustained use. We conducted a systematic three-round Delphi study through the Internet. Both national and international experts (from the fields of health promotion, health psychology, ecommunication, and technical Web design) and potential users were invited via email to participate. During this study an extensive list of factors potentially related to optimal development of health-related websites was identified, by focusing on factors related to layout, general and risk information provision, questionnaire use, additional services, and ease of use. Furthermore, we assessed the extent to which experts and potential users agreed on the importance of these factors. Differences as well as similarities among experts and potentials users were deduced. In total, 20 of 62 contacted experts participated in the first round (32\% response rate); 60 of 200 contacted experts (30\% response rate) and 210 potential users (95\% response rate) completed the second-round questionnaire, and 32 of 60 contacted experts completed the third round ( $53 \%$ response rate). Results revealed important factors consented upon by experts and potential users (e.g., ease of use, clear structure, and detailed health information provision), as well as differences regarding important factors consented upon by experts (e.g., visual aids, self-monitoring tool, and iterative health feedback) or by potential users only (e.g., bread crumb navigation and prevention of receiving spam). This study is an important first step in determining the agreed-upon factors that should be taken into account when developing online health promotion programs. The public health impact of these programs will be improved by optimizing the development process in line with these factors. 


\section{INTRODUCTION}

Worldwide, more and more people are accessing the Internet in search of health-related information (Bansil et al., 2006). It is estimated that globally a minimum of nearly seven million health-related Internet searches are conducted daily (Eysenbach \& Kohler, 2003). Since Internet penetration rates are still expanding, with currently almost two billion people having access to the Internet, the number of health-related searches is also expected to increase (Internet World Stats, 2013). Therefore, the Internet is a promising channel for offering a broad range of health-related information, such as background information on health, treatment information, medication information, and health behaviour information (Bansil et al., 2006).

Due to this high level of accessibility and its potential to reach large numbers of people (Internet World Stats, 2013), the Internet has also become a popular medium in the field of health promotion for offering tailored and targeted health promotion programs (Brug et al., 2005; Ritterband et al., 2003). As a consequence, in recent years positive effects of online interventions applying computer tailored techniques have been reported addressing different health behaviours (Krebs et al., 2010), such as physical activity (Van den Berg et al., 2007; Vandelanotte et al., 2007), nutrition (Brug et al., 1998; Neville et al., 2009), smoking cessation (Borland et al., 2004; Dijkstra, De Vries, \& Roijackers, 1998; Myung et al., 2009; Strecher, 1999), and alcohol consumption (Riper et al., 2008; White et al., 2010). Although these tailored interventions are very promising and have proven to be effective, actual reach is failing to live up to the high expectations (Cook et al., 2000; Crutzen, 2009; De Nooijer et al., 2005; Evers et al., 2005; Eysenbach, 2005; Glasgow et al., 2007). Since the public health impact of interventions is determined not only by their efficacy but also by their levels of exposure in the target group (De Vries \& Brug, 1999; Glasgow et al., 1999), it is imperative to put effort into optimizing the level of exposure to Internet-delivered lifestyle interventions.

Successful exposure is partly defined by the level of first-time access of the inter- 
vention, also referred to as first use or adoption (Crutzen, 2009; Glasgow et al., 1999; Rogers, 1995). Besides first use, prolonged use of the intervention is essential. That is, engaging users in the intervention for a substantial amount of time fosters their knowledge of its content and involvement in its effective components, which consequently increases the chances of health behaviour change (Danaher et al., 2006; Eysenbach, 2005). Since health behaviour change is a complex and continuous process, achieving sustained behaviour change depends on both the intensity of the intervention and the number of times the intervention is visited (Crutzen, de Nooijer, Candel, et al., 2008; Verheijden et al., 2007). Due to this high dose-response relationship, ensuring adherence or sustained use of the program is essential to further maximize its effect on subsequent health behaviour change (Christensen et al., 2009; Norman et al., 2007). Both prolonged and sustained use of the intervention can be influenced not only by user characteristics (e.g., demographic characteristics and motivation to use the intervention (Brouwer et al., 2008; Eysenbach, 2005; Moorman \& Matulich, 1993)), but also by specific strategies to increase adherence (e.g., sending periodic reminders (Brouwer, Kroeze, et al., 2011; Fry \& Neff, 2009)) and by intervention characteristics (e.g., appearance and content of the intervention (Brouwer, Kroeze, et al., 2011; Ritterband et al., 2009)).

As online behaviour change interventions are delivered to the public by using a website or Web-based program, development of the website or program refers to composition of the actual intervention, and requires careful composition of the complete website or program it is embedded in. The website as a mode of delivery is described in the Internet intervention model as consisting of 8 main areas (Ritterband et al., 2009): appearance (e.g., the organization of information), behavioural prescriptions (e.g., instructions on how to achieve behaviour change), burdens (e.g., poor navigation applications), content (e.g., treatment information), delivery (e.g., use of animations, audio, or graphics), message (e.g., credibility and likability of the source), participation (e.g., degree of interaction or the use of rewards), and assessment (e.g., measuring needs of users or adjusting content to personal wishes). Adjusting these characteristics enables tailoring of the website to special needs of the population under consideration. All individual characteristics in these areas should therefore be kept in mind while developing the program.

The primary characteristics of an intervention are determined during its development process. According to diffusion theory and social marketing principles (Cugelman et al., 2011; Rogers, 1995), program development is one of the main processes to influence adoption rates of a new product (e.g., a new website or online intervention). Besides including experts in the development process, it should also be done in accordance with the needs and wishes of the target group (Cugelman et al., 
2011). By considering and incorporating the opinions of potential future users, involvement is expected to increase, consequently resulting in increased appreciation of the intervention, lower levels of attrition, and higher levels of prolonged and sustained use (Glasgow et al., 2004). A new intervention should, therefore, be developed in close collaboration with the target group.

Although many studies have investigated the effectiveness and appreciation of numerous components of websites that deliver health behaviour interventions, results have been inconclusive. Some studies recommend using a multimedia approach (Cunningham, Rapee, \& Lyneham, 2006) or the use of interactive tools, such as surveys, quizzes, and games, whereas other studies seem to contradict these findings (Ritterband et al., 2003). Systematically studying different elements of websites is a very extensive and time-consuming process. Even though some elements of websites should be incorporated at all times, such as accurate and comprehensible information (Eysenbach \& Kohler, 2002), a lot of effort should still be put into making other elements operational (Ritterband et al., 2009).

To date, studies on development of health-related websites have not included perspectives of both experts and users. Hence, this study was a first attempt to identify which elements on health-related websites are perceived as necessary and preferable by these two groups. The current study thus included both experts in the fields of health promotion, health psychology, e-communication, and technical Web design and potential future users. By including elements that are perceived as important by both groups while developing the website, developers may optimize exposure rates of the program (Cugelman et al., 2011). Furthermore, involving different groups of experts will lead to more diverse information regarding health communication and behaviour change as well as technical information regarding website development. To identify the potential factors that are related to optimal development of healthrelated websites, we conducted a three-round Delphi study. The specific aim of the study was to identify factors that are associated with optimal development of healthrelated websites. Besides identifying these factors, we investigated the degree to which experts and potential future users agreed on the importance of the factors.

\section{METHODS}

We conducted a three-round Delphi study through the Internet. Due to its systematic nature, a Delphi study is considered to be an appropriate method to derive consensus on health-related issues for which scientific evidence is incomplete or scarce by involving a representative panel of experts (Adler \& Ziglio, 1996). Also, because after each round feedback on group results is provided, the iterative approach allows participants to adjust their opinions when needed. Finally, the structure of the study 
guarantees anonymity of the participants, thereby preventing conformity biases (Crutzen, de Nooijer, Brouwer, et al., 2008; Elfeddali, Bolman, Mesters, Wiers, \& de Vries, 2010).

The current Delphi study consisted of two sub studies. The first sub study (study $1 \mathrm{~A}$ ), including only experts, consisted of three rounds. The first round aimed at providing a list of potential factors related to optimal development of health-related websites by means of an open-ended questionnaire. Next, for the second round, experts were invited to rate the importance of all factors identified in the first round by using a structured questionnaire. Finally, a third round enabled experts to re-evaluate their opinions by providing controlled feedback regarding group mean scores, thereby producing consensus. The second sub study (study 1B) included only potential future users and consisted of one round. This round resembled the second round of the first sub study and allowed users to rate the importance of those factors that were identified by experts in the first round by using the same structured questionnaire. We compared second-round results from both studies in order to identify potential differences between experts and potential users regarding the importance they placed on factors related to optimal design.

\section{STUDY $1 A$ - EXPERTS}

\section{First round}

Procedure and participants

For the first round, we selected experts from the fields of health promotion, health psychology, e-communication, and technical Web design to obtain a variety of insights from researchers with both theory-based and more practice-based backgrounds. Invited experts with a theory-based background were all first or second authors on scientific papers in the field of eHealth and eHealth promotion published between 2000 and 2009. We used database searches in PsychINFO and Medline to identify experts and to examine reference lists from related papers, book chapters, review studies, and conference abstracts. We selected experts with a practice-based background on the basis of their publications, but also by approaching our own network and by asking responding experts to provide names of important experts in the field.

This resulted in a list of 62 experts who were invited by email to participate in all three rounds of the Delphi study (Table 4.1). The email contained detailed information on the goal and study procedure, as well as a link referring them directly to the first-round questionnaire. Non-responders received a reminder email after the 3week response period expired. A total of 20 experts (32\% response rate) responded to the invitation. 
Table 4.1. First-, second- and third-round rates of responses of experts

\begin{tabular}{lcccccc}
\hline \multirow{2}{*}{ Field } & \multicolumn{2}{c}{ Response round 1 } & \multicolumn{2}{c}{ Response round 2 } & \multicolumn{2}{c}{ Response round 3 } \\
\cline { 2 - 6 } & Invitations & Response & Invitations & Response & Invitations & Response \\
\cline { 2 - 7 } Health promotion & 16 & $7(44 \%)$ & 60 & $18(30 \%)$ & 18 & $10(56 \%)$ \\
Health psychology & 16 & $6(38 \%)$ & 60 & $14(23 \%)$ & 14 & $8(57 \%)$ \\
E-communication & 15 & $5(33 \%)$ & 40 & $12(30 \%)$ & 12 & $5(42 \%)$ \\
Web-design & 15 & $2(13 \%)$ & 40 & $16(40 \%)$ & 16 & $9(56 \%)$ \\
\hline
\end{tabular}

\section{Questionnaire}

The first-round questionnaire consisted of 7 open-ended questions. The questions pertained to different subjects related to the development of health-related websites: (1) optimal layout, (2) type of general information provided, (3) type of health risk information provided, (4) ease of use, (5) use of visual aids, and (6) additional information provided. Health-related websites often provide questionnaires to allow visitors the opportunity to assess their own current health status. To provide an accurate update of their health status these questionnaires often tend to be extensive and therefore sensitive to early dropout. Therefore, we also included one open-ended question to gain more insight into factors that contribute to completion of the questionnaires often provided on health-related websites (7). Health-related websites were defined as websites aiming at assisting people to adopt a healthier lifestyle, by offering them important and diverse information regarding health and health-related behaviours (e.g., physical activity, smoking, alcohol consumption, and nutrition).

\section{Data analysis}

Responses of experts were analysed, resulting in an extensive list of potential factors related to optimal development of health-related websites. Two researchers independently listed all unique factors and combined similar responses into 1 factor. For those factors on which no agreement was obtained, a third researcher was approached to give a decisive answer.

\section{Second round}

\section{Procedure and participants}

Experts participating in the first round were also invited to participate in the second round. We selected an additional 180 experts by means of the same strategies used for the first round. A total of 200 experts received an email inviting them to participate in the second and third rounds. The invitation contained a link that directed experts to the second-round questionnaire. Non-responders received two reminder 
emails: after 3 and after 5 weeks. A total of 60 experts (30\% response rate) responded to the invitation (Table 4.1).

\section{Questionnaire}

The second-round questionnaire was composed based on the factors identified by experts in the first round. This resulted in a questionnaire consisting of 85 structured questions. Experts were asked to rate these factors on a 7-point Likert scale ranging from 1 (not at all important) to 7 (extremely important).

\section{Data analysis}

The closed-ended questions were analysed following the standards for analysing data for a Delphi study, by calculating median scores, also referred to as the $50^{\text {th }}$ percentile score, to determine the importance of the various factors. Furthermore, the interquartile range (IQR) was calculated to assess the degree of agreement between the experts on the importance of the factors (Brouwer et al., 2008; Crutzen, de Nooijer, Brouwer, et al., 2008; De Vet, Brug, De Nooijer, Dijkstra, \& De Vries, 2005). The IQR represents the distance between the $25^{\text {th }}$ and the $75^{\text {th }}$ percentiles, with a small value indicating a higher degree of agreement. An IQR smaller than 1 is considered to indicate good consensus on a 7-point scale as used in the present study and means that more than $50 \%$ of all cases fall within 1 point of one another (Linstone \& Turoff, 1975). To deduce those factors that were considered to be either very or extremely important by the majority of experts $(I Q R<1)$, the cut-off point for importance was a median score of $\geq 6$.

\section{Third round}

\section{Procedure and participants}

All experts participating in the second round $(n=60)$ were invited to participate in the third and final Delphi round, using the same procedure as in the previous round. A total of 32 experts (53\% response rate of second-round participants) responded to the invitation (Table 4.1).

\section{Questionnaire}

The third-round questionnaire was an adapted version of the second-round questionnaire, containing only those factors ( $n=27$ ) on which no consensus was obtained during the second round (IQR $>1)$. In line with the Delphi method, additional feedback on second-round group results (median and IQR) was provided, enabling experts to rerate their answers.

Data analysis 
The degree of agreement and consensus among experts was measured by computing median scores and IQRs.

\section{STUDY IB - POTENTIAL FUTURE USERS}

\section{Second round}

Procedure and participants

Potential future users were recruited through an independent, commercial Dutch Internet research panel (FlyCatcher, 2011). From this panel, consisting of approximately 20,000 members, a sample of 220 members was invited to participate in this study. Potential users were invited to take part only in the second round of this Delphi study, since the main goal was to compare users' opinions with experts' opinions and not to force consensus among users on the different factors. Respondents from this panel were rewarded for their participation in this study in accordance with the standards of the consumer panel (approximately $€ 5$ ). All participants received an email invitation informing them about the goal and content of the study. The invitation also contained a link that directed participants to the questionnaire. A total of 210 potential future users (95.5\% response rate) responded to the invitation (Table 4.2).

\section{Questionnaire}

The questionnaire assessed demographics such as gender, age, ethnicity, education level, and income. In addition, participants were asked to indicate how often they used the Internet for work or study purposes (1, [almost] never; 6, every day), for private use (1, [almost] never; 6 , every day), and for finding information on healthrelated topics ( 1 , never; 6 , very often). For the questions assessing Internet use for work and private purposes, new categories were composed: frequent user ( $>3$ times a week), average user (every week, but not $>3$ days a week), and infrequent user ( $<1$ a week). Furthermore, the questionnaire contained all factors identified by experts in the first round of study 1 a, resulting in a questionnaire consisting of 92 questions. Potential future users were asked to rate the importance of all factors on a 7-point Likert scale ranging from 1 (not at all important) to 7 (extremely important).

\section{Data analysis}

Data were analysed according to the same principles used for the second round of study 1 a, by calculating median scores and IQRs. Differences in consensus between the expert group and potential future users were analysed using Wilcoxon signed rank sum tests. Similarities and differences in second-round results between experts and 
potential future users were further analysed by using multivariate analysis of variance.

\section{Table 4.2. Demographic profile of potential future users}

\begin{tabular}{|c|c|c|}
\hline \multirow[t]{2}{*}{ Demographic characteristics } & \multicolumn{2}{|c|}{$n=210$} \\
\hline & $\%$ & $\mathrm{~N}$ \\
\hline \multicolumn{3}{|l|}{ Age (19-65), $46.49(S D=47.00)$} \\
\hline \multicolumn{3}{|l|}{ Gender } \\
\hline Male & 51 & 106 \\
\hline Female & 49 & 104 \\
\hline \multicolumn{3}{|l|}{ Ethnicity } \\
\hline Native & 98 & 206 \\
\hline Non-native & 2 & 4 \\
\hline \multicolumn{3}{|l|}{ Education } \\
\hline Low & 11 & 24 \\
\hline Medium & 59 & 123 \\
\hline high & 30 & 63 \\
\hline \multicolumn{3}{|l|}{ Income } \\
\hline Less than average & 29 & 61 \\
\hline Average & 26 & 54 \\
\hline More than average & 45 & 94 \\
\hline \multicolumn{3}{|l|}{ Internet use: work or study } \\
\hline Frequent user & 55 & 116 \\
\hline Average user & 12 & 26 \\
\hline Infrequent user & 10 & 20 \\
\hline Not applicable & 23 & 48 \\
\hline \multicolumn{3}{|l|}{ Internet use: private use } \\
\hline Frequent user & 96 & 202 \\
\hline Average user & 3 & 6 \\
\hline Infrequent user & 1 & 2 \\
\hline \multicolumn{3}{|l|}{ Internet use: health purposes } \\
\hline Frequent user & 21 & 45 \\
\hline Average user & 69 & 144 \\
\hline Infrequent user & 10 & 21 \\
\hline
\end{tabular}

\section{RESULTS}

\section{Study $1 A$ - experts}

We used experts' responses from the first round to compose the questionnaire for the second round, and results of the first round are therefore shown as question items. We grouped items with regard to layout, general information content, health risk information content, ease of use, questionnaire completion, visual aids, and additional services. This resulted in a list of 85 factors thought to be related to optimal development of health-related websites. There were 10 factors in the layout category, 11 in general information content, and 16 in health risk information content. A total of 
18 factors were mentioned as facilitating ease of use. The remaining factors referred to questionnaire completion (11), visual aids (6), and additional services (13). An overview of all results is given in Appendix 1.

\section{Consensus}

During the second round, consensus was reached (IQR $\leq 1)$ on 57 factors. After experts rerated their answers during the third round, aided by feedback on secondround group results, consensus was obtained on another 6 factors. In total consensus was obtained on $74 \%$ of all factors.

Table 4.3. Median scores for important factors related to development of health-related Internet sites on which consensus was obtained by experts, potential users or both

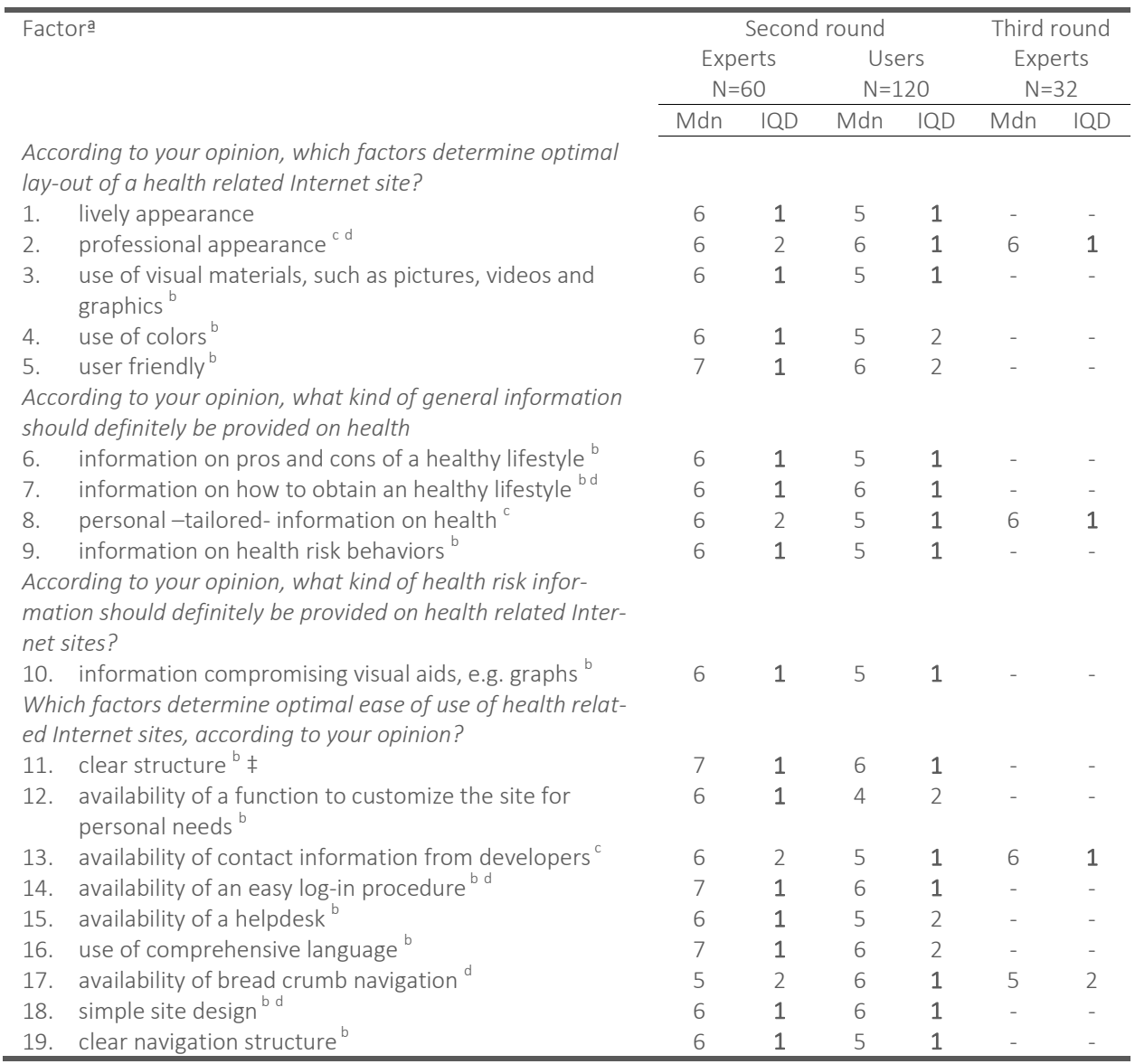


Table 4.3. continued: Median scores for important factors related to development of health-related Internet sites on which consensus was obtained by experts, potential users or both

\begin{tabular}{|c|c|c|c|c|c|c|}
\hline \multirow[t]{3}{*}{ Factor $\underline{a}$} & \multicolumn{4}{|c|}{ Second round } & \multirow{2}{*}{\multicolumn{2}{|c|}{$\begin{array}{l}\text { Third round } \\
\text { Experts } \\
\mathrm{N}=32\end{array}$}} \\
\hline & \multicolumn{2}{|c|}{$\begin{array}{l}\text { Experts } \\
\mathrm{N}=60\end{array}$} & \multicolumn{2}{|c|}{$\begin{array}{l}\text { Users } \\
\mathrm{N}=120\end{array}$} & & \\
\hline & Mdn & IQD & Mdn & IQD & Mdn & IQD \\
\hline \multicolumn{7}{|l|}{$\begin{array}{l}\text { According to your opinion, which factors determine whether } \\
\text { visitors complete questionnaires provided on health related } \\
\text { Internet sites? }\end{array}$} \\
\hline $\begin{array}{l}\text { 20. provide information on relevance of questionnaire } \\
\text { completion }^{\text {b }}\end{array}$ & 6 & 1 & 5 & 1 & - & - \\
\hline 21. provide information on personal benefits of completion & 6 & 1 & 5 & 1 & - & - \\
\hline 22. provide a progress bar ${ }^{\text {b d }}$ & 6 & 1 & 6 & 1 & - & - \\
\hline $\begin{array}{l}\text { 23. provide clearly structured questionnaire by using clear } \\
\text { heads and subheads }{ }^{\text {bd }}\end{array}$ & 6 & 1 & 6 & 1 & - & - \\
\hline $\begin{array}{l}\text { 24. provide guarantee that completion will not result in the } \\
\text { receipt of spam }{ }^{d}\end{array}$ & 6 & 2 & 7 & 1 & 5 & 1 \\
\hline $\begin{array}{l}\text { 25. provide opportunity to stop completion and proceed at } \\
\text { a later point in time }{ }^{b d}\end{array}$ & 6 & 1 & 6 & 1 & - & - \\
\hline 26. use of original questions ${ }^{d}$ & 4 & 1 & 6 & 1 & - & - \\
\hline \multicolumn{7}{|l|}{$\begin{array}{l}\text { What additional services should be provided on health relat- } \\
\text { ed Internet sites, according to your opinion? }\end{array}$} \\
\hline $\begin{array}{l}\text { 27. provide a self-monitoring tool to monitor personal } \\
\text { health behavior change }\end{array}$ & 6 & 1 & 5 & 2 & - & - \\
\hline provide a privacy statement $^{\mathrm{c}}$ & 6 & 2 & 5 & 2 & 6 & 1 \\
\hline $\begin{array}{l}\text { 29. provide the opportunity to print or download relevant } \\
\text { information }^{\text {b }}\end{array}$ & 6 & 1 & 5 & 1 & - & - \\
\hline 30. provide a search engine & 5 & 1 & 6 & 1 & - & - \\
\hline 31. provide the opportunity for regular revisits to the site ${ }^{d}$ & 6 & 1 & 5 & 1 & - & - \\
\hline $\begin{array}{l}\text { 32. provide iterative feedback during revisits to assess } \\
\text { users against their own previous performances }{ }^{\text {b }}\end{array}$ & 6 & 1 & 5 & 2 & - & - \\
\hline
\end{tabular}

\footnotetext{
${ }^{a}$ Only experts were included in the third round. Statements on which consensus was obtained in the second round were excluded from the third round and results are therefore missing. Only factors on which consensus was obtained either by experts, users or both are displayed (interquartile range $\leq 1$ ).

${ }^{b}$ Interquartile range.

c Not applicable.

${ }^{d}$ Factors on which consensus was obtained by only by potential users.

${ }^{b}$ Factors on which consensus was obtained by both experts and potential users.
}

\section{Importance}

Experts identified 32 factors as being very or extremely important (median $\geq 6$ ): (1) layout, with 5 factors ( $50 \%$ of layout-related factors, e.g., professional appearance and use of colour), (2) general information provision, with 4 factors (36\% of general information-related factors, e.g., information on pros and cons of a healthy lifestyle, and tailored information on health), (3) health risk information provision, with 1 factors (6\% of health risk-related factors, e.g., information on skills that help to decrease 
health risks and personal advice on how to decrease health risks), (4) ease of use, with 10 factors (56\% of factors related to ease of use, e.g., availability of an easy log-in procedure and a clear navigation structure), (5) questionnaire completion, with 6 factors ( $55 \%$ of factors related to completion, e.g., provision of a progress bar and provision of an option for partial completion), and (6) additional services, with 5 factors (38\% of factors related to additional services, e.g., provision of a self-monitoring tool and iterative feedback).

Although consensus was obtained on 3 factors relating to the provision of visual aids (e.g., provision of cartoons, pictures, and graphical representations of relevant information), these factors had respective median scores of 4, 5, and ,5 and thus were not considered as extremely or very important. Combining the results on importance and obtained consensus, we can conclude that consensus (IQR $\leq 1)$ was obtained on the importance (median $\geq 6$ ) of 24 factors (Table 4.3).

\section{Study 1B - potential future users}

Concensus

Potential future users reached consensus (IQR $\leq 1)$ on 60 of the 85 factors (71\%). An overview of all results is given in Appendix 1.

\section{Importance}

A total of 17 factors were identified as being very or extremely important (median $\geq$ 6). These factors were mapped into different categories: (1) layout, with 3 factors (30\% of factors related to layout, e.g., limited amount of distractions and user friendliness), (2) general information provision, with 1 factor ( $9 \%$ of factors related to general information; information on how to attain a healthy lifestyle), (3) ease of use, with 7 factors (39\% of factors related to ease of use, eg, availability of an easy log-in procedure and use of comprehensive language), (4) questionnaire completion, with 3 factors ( $55 \%$ of factors related to questionnaire completion, eg, provision of a progress bar and provision of an option for partial completion), and (5) additional services, with 5 factors (38\% of factors related to additional services, e.g., provision of a self-monitoring tool and iterative feedback).

Although consensus was obtained on 14 factors relating to ease of use (e.g., information on skills that help to decrease health risks, and personal advice on how to decrease health risks), all these factors had median scores of 5 and were not considered to be extremely or very important. Furthermore, all factors related to provision of visual aids had median scores $<6$, even though consensus was obtained on all 6 factors. Combining the results on importance and obtained consensus, we can conclude that consensus $(I Q R \leq 1)$ was obtained on the importance (median $\geq 6$ ) of 11 
factors (Table 4.3).

Similarities and differences between experts and potential future users

The results of the second round revealed that experts agreed on the importance of 24 factors, whereas the majority of potential future users agreed on the importance of 11 factors (median $\geq 6 ; \mid Q R \leq 1$ ). The statistical tests showed a not significant ( $z=-$ $.262, P=.29$ ) difference between the overall level of consensus obtained in the two groups. Experts and potential future users both agreed on the importance of $7 \mathrm{fac}$ tors: (1) general information, with 1 factor (the availability of information on how to attain a healthy lifestyle), (2) ease of use, with 3 factors (clear structure, easy log-in procedure, simple site design), and (3) questionnaire completion, with 3 factors (provision of a progress bar, a clearly structured questionnaire, and an option for partial completion).

Contrary to these similarities in perceived importance, experts and potential future users' opinions differed on 22 factors, significantly so on 19 factors (Table 4.4). The multivariate analysis of variance found a significant effect of group on the importance of factors related to optimal development $\left(F_{1,268}=95.95, P<.001, R^{2}=.09\right.$. Univariate $F$ tests revealed that experts rated a lively appearance, the use of visual aids, the use of colour, user friendliness (optimal layout), information in the form of visual aids (health risk information), opportunities for customizing the site, availability of a helpdesk, use of comprehensive language, simple site design, clear navigation structure (optimal ease of use), provision of relevance and personal benefits of completing the questionnaire (questionnaire completion), provision of a self-monitoring tool, option for printing and downloading information, stimulation of revisits, and iterative feedback (additional services) as significantly more important factors than did potential future users. Conversely, potential future users rated the availability of bread crumb navigation (optimal ease of use), the provision of a guarantee that questionnaire completion will not result in receiving spam, and usage of original questions in questionnaires (questionnaire completion) as more important than did experts.

Table 4.4. Univariate $\boldsymbol{F}$-tests for differences in rating the importance of a factor between experts and users

\begin{tabular}{|c|c|c|c|c|}
\hline \multirow[t]{2}{*}{ Factor } & $\begin{array}{c}\text { Mean } \\
\text { experts }\end{array}$ & $\begin{array}{l}\text { Mean } \\
\text { users }\end{array}$ & $F$ & $\eta^{2}$ \\
\hline & $N=60$ & $N=210$ & & \\
\hline \multicolumn{5}{|l|}{ Optimal lay-out } \\
\hline lively appearance & $5.48(0.83)$ & $5.01(0.98)$ & $11.37^{* * *}$ & .04 \\
\hline professional appearance & $5.87(0.97)$ & $5.62(0.81)$ & 3.84 & .01 \\
\hline
\end{tabular}


Table 4.4. continued: Univariate $\boldsymbol{F}$-tests for differences in rating the importance of a factor between experts and users

\begin{tabular}{|c|c|c|c|c|}
\hline \multirow[t]{2}{*}{ Factor } & $\begin{array}{l}\text { Mean } \\
\text { experts }\end{array}$ & $\begin{array}{l}\text { Mean } \\
\text { users }\end{array}$ & $F$ & $\eta^{2}$ \\
\hline & $N=60$ & $N=210$ & & \\
\hline $\begin{array}{l}\text { use of visual materials, such as pictures, vid- } \\
\text { eos and graphics }\end{array}$ & $5.98(0.89)$ & $5.21(0.98)$ & $30.23 * * *$ & .10 \\
\hline use of colors & $5.55(0.89)$ & $5.00(1.05)$ & $13.85^{* * *}$ & .05 \\
\hline user friendly & $6.68(0.57)$ & $6.09(0.89)$ & $24.08 * * *$ & .08 \\
\hline \multicolumn{5}{|l|}{ General information } \\
\hline $\begin{array}{l}\text { information on pros and cons of a healthy } \\
\text { lifestyle }\end{array}$ & $5.53(0.91)$ & $5.36(0.97)$ & 1.58 & .01 \\
\hline $\begin{array}{l}\text { information on health risk behaviours } \\
\text { Health risk information }\end{array}$ & $5.60(0.81)$ & $5.43(0.96)$ & 1.59 & .01 \\
\hline $\begin{array}{l}\text { information compromising visual aids, such } \\
\text { as graphs }\end{array}$ & $5.71(1.09)$ & $4.88(1.06)$ & $28.53 * * *$ & .10 \\
\hline \multicolumn{5}{|l|}{ Optimal ease of use } \\
\hline $\begin{array}{l}\text { availability of a function to customize the } \\
\text { site for personal needs }\end{array}$ & $5.78(0.98)$ & $3.92(1.36)$ & $97.63^{* * *}$ & .27 \\
\hline availability of a helpdesk & $5.68(0.89)$ & $5.04(1.06)$ & $18.09 * * *$ & .06 \\
\hline use of comprehensive language & $6.50(0.70)$ & $6.01(0.91)$ & $14.61^{* * *}$ & .05 \\
\hline availability of bread crumb navigation & $5.05(1.19)$ & $5.63(0.97)$ & $15.22 * * *$ & .05 \\
\hline simple site design & $6.02(0.89)$ & $5.65(0.89)$ & $7.88^{* *}$ & .03 \\
\hline clear navigation structure & $6.57(0.75)$ & $2.18(0.95)$ & $1084.30 * *$ & .80 \\
\hline \multicolumn{5}{|l|}{ Questionnaire completion } \\
\hline $\begin{array}{l}\text { provide information on relevance of ques- } \\
\text { tionnaire completion }\end{array}$ & $5.77(0.89)$ & $5.51(0.89)$ & $3.92 *$ & .01 \\
\hline $\begin{array}{l}\text { provide information on personal benefits of } \\
\text { completion }\end{array}$ & $5.78(0.92)$ & $5.34(0.98)$ & $9.95 * *$ & .04 \\
\hline $\begin{array}{l}\text { provide guarantee that completion will not } \\
\text { result in the receipt of spam }\end{array}$ & $5.87(1.16)$ & $6.29(0.95)$ & $8.44^{* *}$ & .03 \\
\hline use of original questions & $4.53(1.19)$ & $5.59(1.01)$ & $46.50 * * *$ & .15 \\
\hline \multicolumn{5}{|l|}{ Additional services } \\
\hline $\begin{array}{l}\text { provide a self-monitoring tool to monitor } \\
\text { personal health behaviour change }\end{array}$ & $5.88(0.85)$ & $4.84(1.21)$ & $39.41^{* * *}$ & .13 \\
\hline $\begin{array}{l}\text { provide the opportunity to print or down- } \\
\text { load relevant information }\end{array}$ & $6.18(0.83)$ & $5.41(1.15)$ & $23.41^{* * *}$ & .08 \\
\hline $\begin{array}{l}\text { provide the opportunity for regular revisits } \\
\text { to the site }\end{array}$ & $6.02(0.89)$ & $5.15(1.13)$ & $29.95 * * *$ & .10 \\
\hline provide iterative feedback during revisits & $5.92(0.79)$ & $5.04(1.07)$ & $34.45 * * *$ & .11 \\
\hline
\end{tabular}

\section{DISCUSSION}

This study identified an extensive list of factors that might contribute to the development of health-related websites. The importance of a selected set of these factors was stressed by both experts and potential users. Developers should therefore attempt to take these factors into account when developing a health-related website. In particular, the provision of information on attaining a healthy lifestyle was emphasized. Furthermore, a clearly structured website with a simple design and the pres- 
ence of an easy log-in procedure were brought up as important factors, which corresponds to results from earlier studies (Brouwer et al., 2008; Eysenbach \& Kohler, 2002). Also, with respect to optimizing questionnaire completion, several important factors were identified. To increase questionnaire completion, websites should offer a progress bar and an option for partial completion. These results are also in line with previous findings (Crutzen, 2010). Since questionnaire length seems to be inversely related to actual participation as well as completion (Galesic \& Bosjnak, 2009), providing an option for partial completion might be a solution to prevent attrition when using extensive questionnaires. Besides factors agreed upon by both groups, results also indicated that the majority of experts and users significantly differed on the importance of several factors. Due to their specific knowledge on theories in their area of expertise, experts should be involved in the development process to ensure scientific input. However, opinions of potential users must not be neglected because, by engaging users in this process, their involvement will increase. This involvement will subsequently increase the chances of obtaining higher levels of appreciation, prolonged and sustained use, and lower levels of attrition (Glasgow et al., 2004).

The recently introduced Internet intervention model (Ritterband et al., 2009) offers an opportunity to classify important factors identified in this study into several main areas that determine how the website is developed and functions. The first area pertains to the appearance of the website, which is one of the first website characteristics visitors are confronted with. Since previous research indicated that more than half of all website visitors are inclined to leave the website within the first 30 seconds (Brouwer et al., 2010), the exterior of the website should be appealing in order to attract sufficient visitor attention and prevent early disengagement. In this study, experts highlighted important factors, such as a lively appearance, the use of colour, and the use of visual aids, as contributing to the appearance of health-related websites. Furthermore, potential users stressed the importance of developing websites with a professional appearance (Eysenbach \& Kohler, 2002). The model also emphasizes behavioural prescription, which refers to the instructions users receive on how to change their lifestyle. In the current study, both experts and potential users emphasized the importance of offering information on how to achieve a healthy lifestyle on health-related websites. Another important area of the website is the size of the burden that actual use of the website entails. Results from this study indicated that both experts and users value the presence of an easy log-in procedure. Furthermore, experts stressed the importance of developing a user-friendly website, to decrease the effort visitors must invest in navigating the website. This also entails a simple site design and a clear navigation structure. Furthermore, potential users indicated they appreciated the presence of bread crumb navigation and a search engine, to ensure 
visible navigation on the website (Tognazzini, 2011). Content that is provided on the website should be accurate, complete, and readable (Eysenbach \& Kohler, 2003; Eysenbach, Powell, Kuss, \& Sa, 2002). In line with these findings, experts agreed on the importance of using comprehensive language on health-related websites. Since health-related websites mainly aim at helping or assisting people to adopt a healthy lifestyle, experts stressed that detailed information on the pros and cons of a healthy lifestyle and on health behaviours in general should be included in the website's content (Brouwer et al., 2008; Eysenbach \& Kohler, 2002). Additionally, potential users indicated the importance of an opportunity to download or print relevant information that is provided on the website. Participation is considered as another important component of the website and focuses on its ability to engage and involve visitors. Providing reinforcement is considered an important strategy to engage visitors and can be a reward for progressing through the website content (Ritterband et al., 2009). The provision of personalized feedback regarding the status of lifestyle behaviours and the opportunity to self-monitor behaviour change can be regarded as a form of reinforcement. In line with this, experts stressed the importance of providing a selfmonitoring tool to allow visitors to assess their current health behaviour status and to track their progress. Finally, assessment refers to the website's ability to adjust the website to specific user needs and wishes. In this study, experts indicated that an opportunity to adjust the website to personal preferences was highly appreciated. Therefore, visitors should have an opportunity to adjust not only the appearance of the website but also its content, corresponding to their needs and wishes. Tailoring is considered to be an appropriate strategy to adjust health information to personal characteristics and preferences (Kreuter et al., 2000). In addition, previous studies indicated that providing personalized, iterative feedback regarding one's health behaviour might in itself stimulate revisits to the program (Brouwer et al., 2008). In line with these previous findings, the majority of experts agreed on the importance of stimulating revisits and using iterative feedback. To adjust information to personal characteristics and specific user needs, questionnaires are often requisite to obtain detailed information on these topics. As stressed in the introduction, these questionnaires often tend to be extensive and therefore sensitive to early dropout. It is therefore imperative to stimulate questionnaire completion in order to optimize adjustment to visitor's needs and wishes. Experts indicated that information on relevance and personal benefit (Crutzen, 2010) of completing a questionnaire should be provided. Potential users indicated that developers should develop original questions and guarantee that questionnaire completion will not result in receiving spam.

The Internet Intervention model emphasizes two additional areas that were also identified in this Delphi study: mode of delivery and the message. Mode of delivery 
refers to ways in which the intervention content can be delivered to visitors. Specific strategies that can be used pertain to the use of animations, audio, video, or testimonials. The message area, on the other hand, focuses on the source and style of the message and addresses issues such as the trustworthiness and expertise of the website developers. Even though several factors pertaining to these areas were identified in the Delphi study, the majority of both experts and potential users did not agree on the importance of these factors.

\section{Limitations}

Several limitations to this study should be considered. First, the development of health-related websites is a very broad topic entailing diverse elements. To obtain detailed information on factors related to each separate element of the development process (e.g., deciding on layout, content, and additional services), the questionnaire was divided into 7 categories. This rather broad setup, which is often inherent to the Delphi process, may have limited the specification of in-depth information. As the Delphi method does not allow for further specification of such factors in later rounds, still more in-depth examination of such factors is required. Second, response rates for the expert study ranged between 33\% and 53\%. Although this range is somewhat low, previous Delphi studies have reported similar response rates (Brouwer et al., 2008; De Vet et al., 2005; Mullen, 2003). Suboptimal response rates in our study might have been a consequence of experts being invited to participate in at least two rounds of the Delphi study. Especially, response rates among experts with a practice-based background, coming from the field of technical Web design, were low during the first round. To account for these suboptimal response rates and to balance the input from the various expert fields, we put additional effort into recruiting these experts for participation in the second round. Finally, to ensure dispersion and coverage of answers, as well as optimal participation rates, it is recommendable for future studies to limit the number of questions used in the Delphi study. Incorporating a small number of questions in the first-round questionnaire may allow experts to give more in-depth input and may increase participation in subsequent rounds, thereby diminishing attrition rates.

\section{Implications}

The currents study is a first step in increasing exposure rates of online health promotion programs. The results of this study need further experimental testing to identify which (combination of) factors ultimately results in the best result. Although the vast number of factors that play a role may hinder the feasibility of a full factorial experi- 
mental design, in-depth experimental studies on the importance of some categories of factors are recommended. 


\section{Chapter 5}

\section{The influence of user characteristics}

and a periodic email prompt on use of an Internet-delivered computer tailored lifestyle intervention 


\section{ABSTRACT}

The Internet is a promising medium for offering tailored and targeted lifestyle interventions applying computer tailored (CT) techniques to the general public. Actual exposure to $\mathrm{CT}$ interventions is not living up to its high expectations, as only a limited proportion of the target group is using these programs. The objective was to investigate exposure to an Internet-delivered, CT lifestyle intervention, targeting physical activity, fruit and vegetable intake, smoking behaviour, and alcohol intake, we focused on three processes: first use, prolonged use, and sustained use. The first objectives were to identify user characteristics that predict initiation of an online CT lifestyle program (first use) and completion of this program (prolonged use). Furthermore, the effect of using a proactive strategy, consisting of periodic email prompts, on program revisits (sustained use) was studied. The research population for this study onsisted of Dutch adults participating in the Adult Health Monitor, offered by the Regional Public Health Services. We used a randomized controlled trial design to assess predictors of first use, prolonged use, and sustained use. Demographics and behavioural characteristics, as well as the strategy used for revisiting, were included as predictors in the model. A total of 9169 participants indicated their interest in the new program and 5168 actually logged in to the program. Participants significantly more likely to initiate one of the CT modules were male, older, and employed, and had a lower income, higher body mass index, and relatively unhealthy lifestyle. Participants significantly more likely to complete one of the CT modules were older and had a higher income and a relatively healthier lifestyle. Finally, using a proactive strategy influenced sustained use, with people from the prompting condition being more likely to revisit the program (OR $=28.92,95 \%$ confidence interval 10.65-78.52; $P<.001$ ). Older, male, and employed participants and those with a lower income, higher body mass index, and a relatively unhealthy lifestyle were more likely to initiate a CT module. Module completers predominantly had a higher income and age. The current program therefore succeeded in reaching those people who benefit most from online lifestyle interventions. However, these people tended to disengage from the program. This underlines the importance of additional research into program adjustments and strategies that can be used to stimulate prolonged program use. Furthermore, sending periodic email prompts significantly increased revisits. Though promising, this effect was modest and needs to be further examined, in order to maximize the potential of periodic email prompting. 


\section{INTRODUCTION}

With a substantial number of people accessing the Internet in search of health-related information (Eysenbach \& Kohler, 2002), the Internet has developed into a popular medium for offering a broad range of specific health information, such as information on health and illness, details on treatmentrelated issues, and information on health promotion practices, such as programs that offer information and advice on lifestyle behaviours (Bansil et al., 2006). Since Internet penetration rates are still expanding, with almost two billion people having access to the Internet (Internet World Stats, 2013), the number of health-related searches is also expected to increase. The Internet is therefore considered to be a promising medium in the field of health promotion for offering tailored and targeted promotion programs to the general public (Brug et al., 2005; Internet World Stats, 2013; Lustria et al., 2009; Ritterband et al., 2003).

As online health-promoting applications provide many opportunities for interactivity, they are particularly suited for implementing interventions that offer immediate feedback and advice to users. In particular, lifestyle interventions applying computer tailored (CT) techniques (Brug et al., 2005; Krebs et al., 2010; Ritterband et al., 2003), addressing health behaviours such as physical activity (Van den Berg et al., 2007; Vandelanotte et al., 2007), fruit and vegetable intake (Brug et al., 1998; Neville et al., 2009), smoking cessation (Borland et al., 2004; Dijkstra, De Vries, \& Roijackers, 1998; Myung et al., 2009; Strecher, 1999), and alcohol consumption (Riper et al., 2008; White et al., 2010) have been shown to have positive effects on health behaviour.

Despite these promising prospects, actual exposure to $\mathrm{CT}$ interventions is not living up to the high expectations (Crutzen, 2009; De Nooijer et al., 2005; Evers et al., 2005; Eysenbach, 2005; Glasgow et al., 2007), as only a (limited) proportion of the target group actually uses these programs (Evers et al., 2005). Earlier studies defined 
exposure as pertaining to three different aspects: accessing the intervention (first use), engaging in the intervention content for a substantial period of time (prolonged use), and revisiting the intervention (sustained use) (Crutzen, 2009). The level of firsttime use of online interventions is generally low, with only small proportions of the potential target population actually accessing the intervention (Crutzen, 2009; Glasgow et al., 1999; Rogers, 1995). Levels of actual engagement in the intervention and of revisits to the intervention are even lower (Brouwer, Kroeze, et al., 2011). Since health behaviour change is a complex process, actual change requires prolonged and sustained commitment to the program to enable optimal support during the change process. Intensive engagement in an intervention session allows processing of the intervention content and involvement in its effective components and is therefore essential (Danaher et al., 2006; Eysenbach, 2005; Strecher et al., 2008). Furthermore, a specific number of repeated visits to an intervention may be imperative, as, due to a high dose-response relation (Crutzen, de Nooijer, Candel, et al., 2008; Verheijden et al., 2007), sustained use of the program is essential to further maximize its effect on subsequent health behaviour change (Christensen et al., 2009; Norman et al., 2007).

To increase adoption rates of online interventions, it is imperative to obtain detailed profiles of those who successfully adopt an intervention (Glasgow et al., 1999; Rogers, 1995). By closely studying characteristics of these first-time users and mapping how they use and re-use the intervention, detailed knowledge can be acquired on intervention adopters. It is important to reach those people most in need of online lifestyle interventions - that is, the people who engage in risky behaviours such as smoking, excessive alcohol use, lack of physical activity, or unhealthy eating patterns. Even though unhealthy lifestyle behaviours are prevalent among the whole population (RIVM, 2012; World Health Organisation, 2011), studies have shown that those who have a lower income and educational level (i.e., a low socioeconomic status) are generally more inclined to have an unhealthy lifestyle (RIVM, 2012; Tu \& Cohen, 2008). Furthermore, even though Internet access rates are increasing among people with a lower socioeconomic status (Tu \& Cohen, 2008), their actual exposure to Internet interventions is lagging (Lorence \& Park, 2008; Spittaels, De Bourdeaudhuij, Brug, et al., 2007; Vandelanotte et al., 2005; Yang et al., 2008). Besides socioeconomic status, other user characteristics are reported to have an influence on adoption of online interventions. Previous studies have pointed out that Internet interventions tend to reach women (Brouwer et al., 2010; Etter, 2005; Harvey Berino et al., 2002; Spittaels \& De Bourdeaudhuij, 2007) and older people (Steyaert \& de Haan, 2007). It is important to gain more insight into characteristics of people who are being reached by the program, but also of people who are left unexposed to the program. These insights can be used to acquire knowledge on the development of further steps to im- 
prove exposure to the program.

Besides focusing on first use of the program, it is also imperative to optimize prolonged use of the program and to study related determinants. Previous studies indicated that tailoring in itself is a strategy to prevent early disengagement from online behaviour change programs (Couper et al., 2010; Steyaert \& de Haan, 2007; Strecher et al., 2008). Furthermore, specific user characteristics such as age, gender, and level of education are related to level of program engagement (Brouwer et al., 2010; Strecher et al., 2008; Verheijden et al., 2007), with women, older people, and those who are more highly educated displaying higher levels of program engagement. Since engagement in the program is an important predictor of revisits to the program (Couper et al., 2010), it is important to map how the program is used and to study determinants of prolonged program use. This obtained knowledge can be used to make specific program adjustments, or to develop specific strategies that can be used to enhance prolonged use of the program.

Online interventions are often offered reactively to the public, implying that a passive approach is used, in which users themselves must act in order to repeatedly benefit from the intervention (Eysenbach, 2005; Prochaska et al., 2001). However, since attaining visitors' loyalty to an intervention over an extended period of time is a very strenuous process (Eysenbach, 2005; Glasgow et al., 2007; Verheijden et al., 2007), efforts should be put into ensuring sustained use by employing more proactive strategies (Danaher et al., 2006). The use of periodic email prompts has been proposed as an effective proactive strategy to boost revisits to interventions aimed at stimulating a healthy lifestyle (Fry \& Neff, 2009). However, most studies merely explored the effectiveness and efficacy of the whole intervention, instead of focusing on the added value of periodic prompting as a separate intervention component. As a consequence, there is too little evidence on the absolute effectiveness of sending periodic prompts.

In the present study, we aimed at answering three questions. First, which user characteristics predict initiation of an online CT program (first use)? Second, which user characteristics predict completion of the online CT program (prolonged use)? Third, what is the effect of using a proactive strategy, consisting of periodic email prompts, on program revisits (sustained use)? We addressed these questions among participants in an online CT intervention aimed at multiple health behaviours: physical activity, fruit and vegetable intake, smoking cessation, and alcohol consumption.

\section{METHODS}

In this randomized controlled trial (RCT) we compared the effect on program revisit of a proactive technique applying a periodic email prompt versus the use of a reactive 
approach. Levels of use and re-use of the program within a 4-month period were studied and linked to specific user characteristics. The current RCT was part of a larger RCT testing the effect of the CT program compared with a control group (Schulz et al., 2011). Only people allocated to the study arm receiving computer tailoring of the larger RCT were included in the current RCT. This study was approved by the Medical Ethics Committee of Maastricht University and the University Hospital Maastricht (NL27235.068.09/MEC 09-3-016) and is described in more detail elsewhere (Schneider et al., 2011; Schulz et al., 2011).

The research population for this study consisted of Dutch adults of the provinces of Zeeland and North-Brabant, which participated in the Adult Health Monitor 2009 (GGD Nederland, 2011). This Monitor is used by all Regional Public Health Services and takes place every 4 years. It serves as a monitoring tool to assess the overall level of health in the Dutch population by approaching a representative sample of the population to fill out a questionnaire assessing different aspects of general health (eg, physical and mental health) and health-related topics (e.g., social and physical environment). Participants had the opportunity to complete a written or an online version of this monitoring questionnaire. The CT program was embedded in the online version and was offered as an additional service to online respondents. Data for the present study were collected from November 2009 to August 2010.

\section{Procedure and participants}

After completing the online version of the Adult Health Monitor, all participants were introduced to the program and were offered the opportunity to receive, free of charge, CT feedback about their current health behaviours, such as physical activity, fruit and vegetable intake, alcohol consumption, and smoking. This program consisted of several modules, one per behaviour, which incorporated questionnaire items and provided feedback on several socio-cognitive determinants of each health behaviour. The content of the program modules was based on programs that have been proven to be effective in RCTs for increasing smoking cessation, promoting the intake of fruit and vegetables, increasing the level of physical activity, and reducing the consumption of alcohol (Brug et al., 1999; Poel et al., 2009; Smeets et al., 2008; Van Keulen et al., 2008).

Participants who were interested in the new program were asked to leave their email addresses. They received an email including an invitation to log in to the CT program with a personal log-in code and password, approximately 3 weeks after completion of the Monitor. By logging in to the program, participants received detailed information on the content and purpose of the study. Subsequently, data on demographics and the five health behaviours obtained through the Monitor were trans- 
ported to the CT program, resulting in a personal overview of individuals' current health behaviour status. If respondents were not adhering to the Dutch public health guidelines set for these behaviours, a module generating CT health advice for changing behaviour was available for each health behaviour.

During a 4-month period after the baseline visit, we monitored use of the intervention. People in the prompting condition were prompted proactively via email 3 months after their baseline visit to revisit the CT program. Revisits to the program were stimulated to provide participants with the opportunity to monitor their own behaviour. During a revisit participants could log in to the program and complete the health risk appraisal questionnaire. Based on their answers, a new personal health overview was composed entailing information on their current health behaviour status, as well as on their status during all previous visits. Improvements, deteriorations, or stability of health behaviours were graphically presented. People in the noprompting condition did not receive any additional prompts and were encouraged only at baseline to revisit the program. Reactions to this email prompt were monitored during a 1-month period.

\section{Randomization}

All included participants were randomly allocated to a prompting condition (receiving additional email prompts) or a no-prompting condition (receiving no additional email prompts). We used a computer software randomization device to determine random allocations at the respondent level.

\section{Content}

\section{CT program}

The CT program used a dual approach to guide people toward behaviour change. The first part consisted of a health risk appraisal and was aimed at increasing participants' awareness of their health behaviour status, by comparing their status to the Dutch public health guidelines set for these health behaviours (ie, being moderately physically active for 30 minutes at least 5 days a week, eating two pieces of fruit per day, eating $200 \mathrm{~g}$ of vegetables per day, not drinking more than one [women] or two [men] glasses of alcohol a day, and not smoking). In this health risk appraisal, feedback messages were used to inform people of their status for each health behaviour and to provide them with additional information on the content of the separate guidelines. These feedback messages were complemented by using graphic representations of traffic lights (Bergstrom et al., 2008), with a green light corresponding to adherence to the guidelines and a red light corresponding to non-adherence. An amber light was used for people who were close to adherence to these guidelines. In case of discrep- 
ancies between current behaviour and the guidelines, people were alerted and directed to the CT modules.

Second, assistance was provided in changing participants' health behaviour by offering five separate CT modules. The content of these modules was based on the Integrated Model for exploring motivational and behavioural change (I-Change Model) (De Vries et al., 2003). The modules used a fixed, gradual approach consisting of four steps, guiding people toward behaviour change. The first step addressed the pros and cons of engaging in the desired behaviour under consideration. The second step focused on the role of significant persons in the direct environment and strategies on how to deal with lack of support and bad role models. The final two steps used planning strategies and were aimed at helping people form preparatory plans to start changing their behaviour (step 3) and coping plans to help them overcome difficult situations and prevent relapse (step 4) after changing their behaviour. Within the modules, all health advice was adapted to individuals' characteristics by considering demographic, behavioural, and cognitive characteristics (De Vries \& Brug, 1999; Dijkstra \& De Vries, 1999; Kreuter et al., 2000). Demographic and behavioural characteristics, such as participants' gender and health behaviour status, were directly obtained through the Adult Health Monitor. Cognitive variables, such as attitude, perceived social influence, self-efficacy, intention, and planning strategies (action plans and coping plans), were assessed by using an additional tailoring questionnaire.

The CT modules were embedded in a website that was especially designed for the current project. This website (http://www.mijngezondgedrag.nl) contained general information considering a healthy lifestyle and the selected health behaviours. Furthermore, the website provided specific information regarding the project, a direct link to the CT program, and information on frequently asked questions. During the study, new information (e.g. advice-supporting messages, recipes, and facts) was structurally added to the website.

\section{Email prompt}

Participants in the prompting condition received an email 3 months after their baseline visit, prompting them to revisit the program (Appendix 2). This email opened with a personalized greeting and reminded people about their first visit to the program. Subsequently, people were invited to revisit the program to obtain information on their health status and to monitor their progress. Participants were also informed of the opportunity to receive additional, iterative health advice on the health behaviour(s) selected at baseline or on a new behaviour. Finally, to facilitate logging in to the program, the email also contained details on personal log-in information (user name and password). The email concluded with greetings from the research team and 
contact information.

\section{Measures}

We collected user characteristics to produce a detailed user profile including information on personal characteristics, health behaviour status, and intention to change their health behaviours.

\section{Personal characterisitcs}

Questions pertaining to personal characteristics included questions on age, gender, educational level, personal net monthly income, work situation, marital status, and native country (Table 5.1).

\section{Health behaviour status}

Health behaviour status consisted of information regarding the five key behaviours. Physical activity was measured by the Short Questionnaire to Assess Health-Enhancing Physical Activity (Wendel Vos et al., 2003), and guideline adherence was calculated following procedures used by Ainsworth et al (Ainsworth et al., 2000). Fruit consumption was measured by using a 4-item food frequency questionnaire assessing weekly fruit and fruit juice intake (RIVM, 2005), and vegetable consumption was measured using a 4-item food frequency questionnaire assessing the weekly amount of consumed boiled or baked vegetables as well as salad or raw vegetables (RIVM, 2005). The consumption of alcohol was measured by the Dutch Quantity-FrequencyVariability Questionnaire (Lemmens et al., 1992). And finally, smoking status was assessed by asking participants whether they smoked, what they smoked (cigarettes, cigars, packets of pipe tobacco) and how much they smoked per day (cigarettes) and per week (cigars or packets pipe tobacco).

\section{Body Mass Index}

Data on participants' height (in centimetres) and weight (in kilograms) were used to calculate their body mass index (BMI). BMI is a heuristic used to estimate the level of body fat, and it is defined by dividing a participant's body weight by the square of the participant's height.

\section{Intention to change}

Intention to change a health behaviour was measured by means of 1 item using an algorithm consisting of 10 stages varying from unawareness to action. This variable was recoded in accordance with an adjusted version of the stages of change concept: immotivation (1), precontemplation (2), contemplation (3), preparation (4), and action 
(5) (Dijkstra, Roijackers, \& De Vries, 1998).

\section{Program evaluation}

After completion of each single module, visitors were asked to evaluate the module by providing an overall grade (1 to 10 ).

\section{Outcomes}

To measure first use of the program, we created a dichotomous variable based on program monitoring data, indicating whether participants initiated a module. Initiating a module was labelled as yes when people filled out the first question of this module. To measure prolonged use, we created a new dichotomous variable to indicate whether participants finished a module. Completion of a module was labelled as yes when people also filled out the final question of the module. To establish sustained use of the program, we created another dichotomous variable, indicating whether participants logged in to the program after baseline. Revisiting of the program was measured by comparing the dates of log-in with the baseline date.

\section{Statistical analyses}

First, we used general descriptive statistics to describe personal characteristics of the participants, as well as the main findings concerning current health behaviour adherence to the public health guidelines. Second, logistic regression analyses were conducted. Initiation of a module and completion of a module $(0=n o / 1=y e s)$ were the dependent variables. Demographics (age, gender, marital status, native country, educational level, work status, and income), BMI, and health behaviour status were included in the model as predictors of initiation. We included the same variables, with addition of intention (measured at the beginning of each CT module), in the model as predictors of completion. Logistic regression analysis was conducted with revisiting of the program $(0=$ no/1 = yes $)$ as the dependent variable. The same demographics, as well as study condition, program evaluation, and initiation and completion of a module, were included in the model as predictors of revisiting of the program. All statistical analyses were done in SPSS version 17.0 (IBM Corporation, Somers, NY, USA).

\section{RESULTS}

\section{Intervention visitor's baseline characteristics}

Demographic characteristics

In total, 3.448 people were allocated to the study arm receiving computer tailoring of the larger RCT and were included in the current RCT. Of all participants, with a mean age of 44 (SD 12.60) years, a little more than half were men, and most had a medium 
to high education level and an average to high monthly income (Table 5.1). Threequarters of all participants were employed and more than half were married. Approximately $2 \%(57 / 3448)$ of all visitors were underweight, whereas more than half had a normal weight. One-third of visitors were slightly overweight and 10.3\% (356/3448) were obese. A randomization check revealed no significant differences between respondents in the prompting and no-prompting condition.

Table 5.1. Characteristics of visitors of an online computer tailored lifestyle intervention

\begin{tabular}{|c|c|}
\hline Characteristics & $\begin{array}{c}\text { Total sample } \\
\mathrm{N}=3448\end{array}$ \\
\hline Age (19-64) & $43.61(S D=12.60)$ \\
\hline \multicolumn{2}{|l|}{ Gender (\%) } \\
\hline Male & 52.8 \\
\hline Female & 47.2 \\
\hline \multicolumn{2}{|l|}{ Education level (\%) } \\
\hline Low & 21.6 \\
\hline Medium & 34.5 \\
\hline High & 42.1 \\
\hline Unknown & 1.8 \\
\hline \multicolumn{2}{|c|}{ Personal net monthly income (in Euro's) (\%) } \\
\hline$<1000$ & 6.5 \\
\hline $1001-1350$ & 6.6 \\
\hline $1351-1750$ & 10.6 \\
\hline $1750-3050$ & 34.0 \\
\hline$>3051$ & 28.0 \\
\hline Unknown & 14.1 \\
\hline \multicolumn{2}{|l|}{ Work situation (\%) } \\
\hline Employed & 75.8 \\
\hline Unemployed & 19.2 \\
\hline Unknown & 5.0 \\
\hline \multicolumn{2}{|l|}{ Marital status (\%) } \\
\hline Married & 60.6 \\
\hline Living together & 15.3 \\
\hline Unmarried & 18.4 \\
\hline Divorced & 4.9 \\
\hline Widow & 0.8 \\
\hline \multicolumn{2}{|l|}{ Native country (\%) } \\
\hline The Netherlands & 95.0 \\
\hline Other & 5.0 \\
\hline \multicolumn{2}{|l|}{$\mathrm{BMI}\left(\mathrm{kg} \mathrm{m}^{-2}\right)(\%)$} \\
\hline$<18.5$ & 1.7 \\
\hline$\geq 18.5$ to $<25$ & 52.1 \\
\hline$\geq 25$ to $<30$ & 36.0 \\
\hline$\geq 30$ & 10.2 \\
\hline \multicolumn{2}{|l|}{ Physical activity (\%) } \\
\hline Compliance & 85.2 \\
\hline Non-compliance & 14.8 \\
\hline Vegetable consumption (\%) & \\
\hline
\end{tabular}


Table 5.1. continued: Characteristics of visitors of an online computer tailored lifestyle intervention

\begin{tabular}{lc}
\hline Characteristics & Total sample \\
& $\mathrm{N}=3448$ \\
Compliance & 32.5 \\
$\quad$ Non-compliance & 67.5 \\
Fruit consumption (\%) & \\
$\quad$ Compliance & 45.0 \\
$\quad$ Non-compliance & 55.0 \\
Smoking behavior (\%) & \\
Compliance & 80.9 \\
Non-compliance & 19.1 \\
Alcohol intake (\%) & \\
Compliance & 71.5 \\
Non-compliance & 28.5 \\
Total number of guidelines (compliance) & \\
0 & 0 \\
1 & 5.9 \\
2 & 19.6 \\
3 & 35.8 \\
4 & 27.1 \\
5 & 10.8 \\
\hline
\end{tabular}

\section{Behavioural characteristics}

Regarding the five health behaviours included in the program, 14.8\% (509/3448) did not comply with the Dutch guidelines of at least 30 minutes of moderately intensive physical activity at least 5 days a week. With regard to fruit and vegetable intake, respectively 54.99\% (1896/3448) and 67.49\% (2327/3448) were not adhering to the Dutch guidelines of at least two pieces of fruit and at least $200 \mathrm{~g}$ of vegetables each day. About one in five visitors indicated to smoke 19.1\% (658/3448), and a little more than a quarter $(981 / 3448,28.5 \%)$ did not comply with the Dutch guidelines for alcohol intake.

\section{First use of the Internet-delivered intervention}

\section{CT module initiation}

A total of 1.338 participants (38.81\%) did not participate in the second part of the program, leaving after receiving their personal health overview. The remaining 2.110 visitors (61.19\%) decided to obtain CT lifestyle advice by initiating one of the five behavioural modules. Of all the visitors who decided to obtain personalized health advice, $13.0 \%(n=275)$ initiated the physical activity module, 35.4\% ( $n=747)$ and $29.4 \%$ $(n=621)$ initiated the vegetable and fruit modules, respectively, 9.1\% ( $n=191)$ initiated the smoking module, and $13.1 \%(n=276)$ initiated the alcohol module (Figure 5.1). 
Figure 5.1. Flowchart of exposure to the $\mathrm{CT}$ intervention: first, prolonged and sustained use

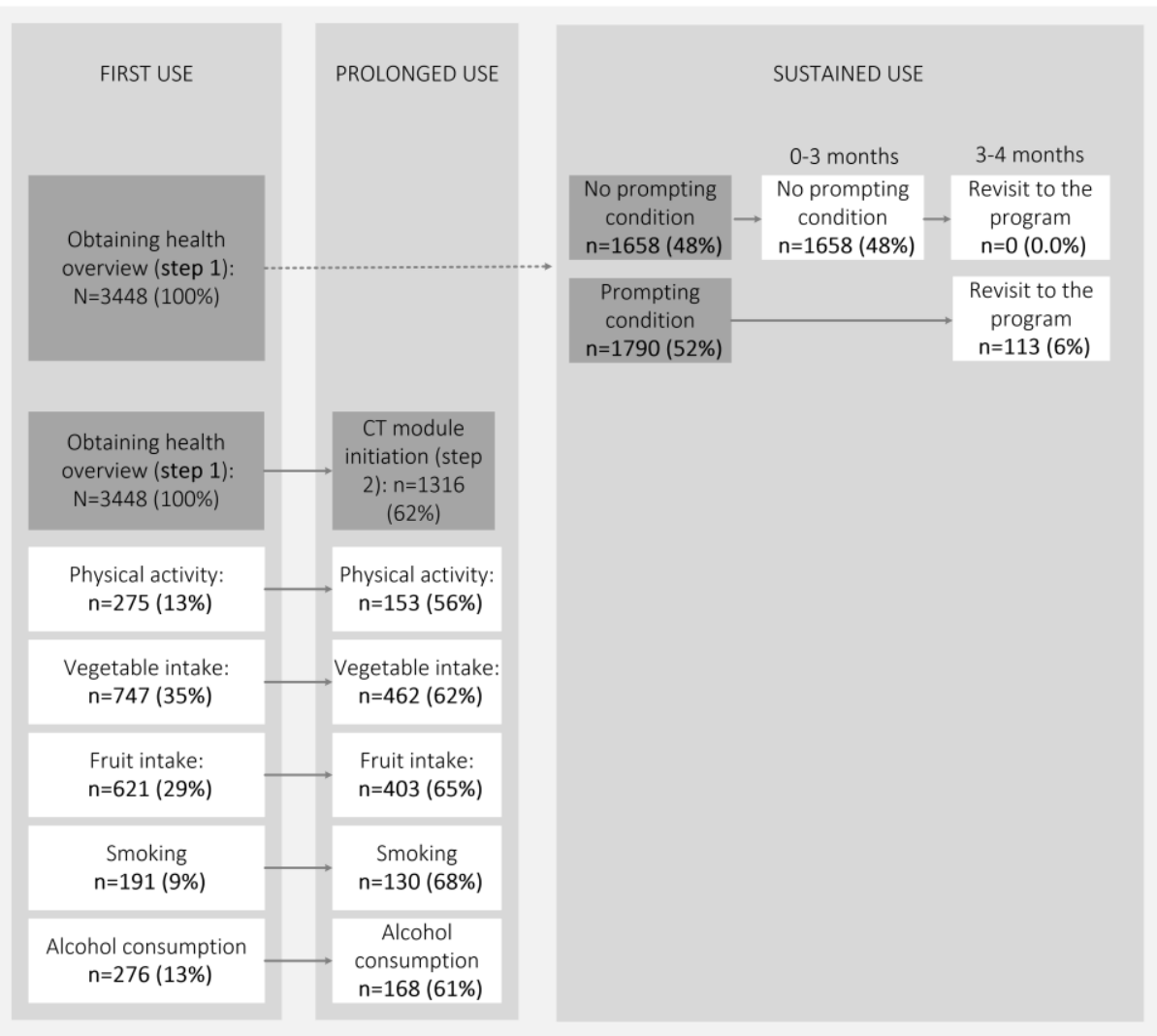

\section{Predictors of CT module initiation}

Results of multiple logistic regression analyses indicated that male, older, and employed participants, and those with a lower income and a higher BMI were more likely to initiate one of the CT modules (Table 5.2). Initiation of a CT module was related to the total number of guidelines people adhered to: those with a relatively unhealthy lifestyle (fewer guidelines complied with) were more likely to initiate one of the CT modules (odds ratio [OR] 0.84, 95\% confidence interval [Cl] 0.77-0.90; $P<.001$ ).

\section{Prolonged use of the Internet-delivered intervention}

\section{CT module completion}

Of all the visitors who initiated one of the CT modules to obtain personalized health advice $(n=2.110), 62.37 \%$ completed a module $(n=1.316)$. A total of 153 of 275 visitors completed the physical activity module (55.6\%), 462/747 (61.9\%) and 403/621 
(64.9\%) completed the vegetable and fruit modules, respectively, 130/191 (68.1\%) completed the smoking module, and 168/276 (60.9\%) completed the alcohol module (Figure 1). All modules were evaluated by visitors upon completion. The physical activity module was graded 7.09 (SD 1.41), vegetable consumption 7.45 (SD 1.02), fruit consumption 7.33 (SD 1.29), smoking 7.35 (SD 1.11), and alcohol consumption 7.39 (SD 1.11).

Table 5.2. Results of multivariate logistic regression analyses on demographic characteristics and behavioural characteristics, with initiating a behavioural computer tailored module as dependent variable $(n=3448)$

\begin{tabular}{|c|c|c|c|}
\hline \multirow[t]{2}{*}{ Characteristics } & \multicolumn{3}{|c|}{ Initiation of a CT behaviour change module } \\
\hline & $\mathrm{OR}^{a}$ & $p$ & $\mathrm{Cl}(95 \%)^{b}$ \\
\hline \multicolumn{4}{|l|}{ Demographic characteristics } \\
\hline Age & 1.35 & $0.00^{*}$ & $1.22-1.49$ \\
\hline \multicolumn{4}{|l|}{ Gender } \\
\hline \multicolumn{4}{|l|}{ Female (reference) } \\
\hline Male & 1.38 & $0.00 *$ & $1.16-1.64$ \\
\hline \multicolumn{4}{|l|}{ Education } \\
\hline \multicolumn{4}{|l|}{ High (reference) } \\
\hline Medium & 1.11 & 0.31 & $0.91-1.34$ \\
\hline Low & 1.07 & 0.59 & $0.83-1.38$ \\
\hline Income & 0.46 & $0.00 *$ & $0.42-0.52$ \\
\hline \multicolumn{4}{|l|}{ Work situation } \\
\hline \multicolumn{4}{|l|}{ Unemployed (reference) } \\
\hline Employed & 1.16 & $0.03 *$ & $1.01-1.33$ \\
\hline \multicolumn{4}{|l|}{ Marital status } \\
\hline \multicolumn{4}{|c|}{ Not in a relationship (reference) } \\
\hline In a relationship & 1.00 & 1.00 & $0.79-1.28$ \\
\hline \multicolumn{4}{|l|}{ Native country } \\
\hline \multicolumn{4}{|c|}{ Other native country (reference) } \\
\hline The Netherlands & 1.38 & 0.12 & $0.92-2.07$ \\
\hline $\mathrm{BMI}$ & 1.03 & $0.02 *$ & $1.01-1.05$ \\
\hline \multicolumn{4}{|l|}{ Behavioural characteristics } \\
\hline Total \# of guideline adherence & 0.84 & $0.00 *$ & $0.77-0.90$ \\
\hline
\end{tabular}

\section{Predictors of CT module completion}

Results of multiple logistic regression analyses indicated that older participants and participants with a higher income were more likely to complete one of the CT modules (Table 3). Participants with a relatively healthy lifestyle (complying with more guidelines) were also more likely to complete one of the CT modules. 
Table 5.3. Results of multivariate logistic regression analyses on demographic characteristics, behavioural characteristics and intention, with completing a behavioural computer tailored module as dependent variable $(n=2110)$

\begin{tabular}{|c|c|c|c|}
\hline \multirow[t]{2}{*}{ Characteristics } & \multicolumn{3}{|c|}{ Initiation of a CT behaviour change module } \\
\hline & $\mathrm{OR}^{a}$ & $p$ & $\mathrm{Cl}(95 \%)^{b}$ \\
\hline \multicolumn{4}{|l|}{ Demographic characteristics } \\
\hline Age & 1.49 & $0.00^{*}$ & $1.33-1.67$ \\
\hline \multicolumn{4}{|l|}{ Gender } \\
\hline \multicolumn{4}{|l|}{ Female (reference) } \\
\hline Male & 1.05 & 0.63 & $0.86-1.29$ \\
\hline \multicolumn{4}{|l|}{ Education } \\
\hline \multicolumn{4}{|l|}{ High (reference) } \\
\hline Medium & $1 . .06$ & 0.67 & $0.80-1.41$ \\
\hline Low & 1.02 & 0.89 & $0.81-1.28$ \\
\hline Income & 1.30 & $0.00^{*}$ & $1.17-1.45$ \\
\hline \multicolumn{4}{|l|}{ Work situation } \\
\hline \multicolumn{4}{|l|}{ Unemployed (reference) } \\
\hline Employed & 0.90 & 0.12 & $0.78-1.03$ \\
\hline \multicolumn{4}{|c|}{ Marital status } \\
\hline \multicolumn{4}{|c|}{ Not in a relationship (reference) } \\
\hline In a relationship & 0.89 & 0.38 & $0.68-1.16$ \\
\hline \multicolumn{4}{|c|}{ Native country } \\
\hline \multicolumn{4}{|c|}{ Other native country (reference) } \\
\hline The Netherlands & 0.87 & 0.54 & $0.57-1.34$ \\
\hline Body Mass Index & 1.00 & 0.94 & $0.97-1.03$ \\
\hline \multicolumn{4}{|l|}{ Sociocognitive characteristics } \\
\hline \multicolumn{4}{|l|}{ Intention } \\
\hline \multicolumn{4}{|l|}{ Action (references) } \\
\hline Immotivation & 1.03 & 0.85 & $0.77-1.37$ \\
\hline Precontemplation & 1.34 & 0.41 & $0.67-2.72$ \\
\hline Contemplation & 0.94 & 0.77 & $0.62-1.42$ \\
\hline Preparation & 1.03 & 0.83 & $0.78-1.36$ \\
\hline \multicolumn{4}{|l|}{ Behavioural characteristics } \\
\hline Total \# of guideline adherence & 1.28 & $0.02 *$ & $1.02-1.24$ \\
\hline
\end{tabular}

${ }^{b}$ Odds ration; ${ }^{b}$ Confidence interval.

$* p<.05$

\section{DISCUSSION}

The current study aimed at investigating the level of exposure to a CT online lifestyle program integrated into the Dutch Adult Health Monitor. We addressed user characteristics that predicted initiation (first use) and completion (prolonged use) of the CT program and tested a proactive strategy using periodic email prompts to enhance revisits to the program (sustained use).

\section{First use}

Since people with a lower income and educational level are generally more inclined to 
have an unhealthy lifestyle (Roos, Johansson, Kasmel, Klumbiene, \& Prattala, 2001; Yang et al., 2008), the current program aimed at reaching especially those people in need of lifestyle improvement. However, in line with previous studies, the majority of participants in our sample had a medium to high educational level, an average income, and a relatively healthy lifestyle (Lorence \& Park, 2008; Spittaels, De Bourdeaudhuij, Brug, et al., 2007; Vandelanotte et al., 2005; Yang et al., 2008). Even though integrating the $\mathrm{CT}$ program into the Monitor environment provided an access point for reaching a substantial proportion of the research population through tailored lifestyle advice, it did not succeed in predominantly reaching the people who benefit most from $\mathrm{CT}$ interventions.

However, taking a closer look at CT module initiation reveals that initiators were significantly older, and likelier to be male, employed, and to have a lower income. Furthermore, participants with a higher BMI and an unhealthier lifestyle were more likely to start with one of the CT modules. Whereas the findings concerning age and gender are largely comparable with those of earlier studies (Brouwer et al., 2010; Spittaels, De Bourdeaudhuij, Brug, et al., 2007; Strecher et al., 2008; Verheijden et al., 2007), the present results are in contrast to previous findings that online health promotion programs tend to be used predominantly by people with a high socioeconomic status and healthy lifestyle (Brouwer et al., 2010; Spittaels, De Bourdeaudhuij, Brug, et al., 2007; Strecher et al., 2008; Verheijden et al., 2007).

The results imply that the current program succeeded in stimulating those people who are expected to benefit most from lifestyle interventions (i.e. the people who engage in risky behaviours, but also people from specific risk groups such as older people and obese people) to initiate one of the CT modules. Embedding the CT program in an existing context, the Adult Health Monitor, may account for these findings. The main objective of the Monitor is to assess different aspects of general health and health-related topics, such as curative care, environment and everyday surroundings, social environment, social safety and violence and nursing and care. Due to this simple focus on assessment and not on health behaviour change, participation may have been appealing to participants who are not primarily interested in behaviour change. However, direct transportation of data from the Monitor to the CT program database allowed for the immediate and effortless composition of a personal health overview. Subsequently, this overview may have increased awareness of people's lifestyle status and might have served as a cue to action to change their lifestyle, explaining the increased interest in the $\mathrm{CT}$ modules, among the unhealthy and low-socioeconomic status participants. 


\section{Prolonged use}

Even though the $\mathrm{CT}$ program succeeded in attracting people with a relatively unhealthy lifestyle and low-socioeconomic status people to initiate one of the CT modules, it fell short in actually engaging these visitors, since module completers predominantly had a higher income, which is in line with previous studies (Brouwer et al., 2010; Spittaels, De Bourdeaudhuij, Brug, et al., 2007; Strecher et al., 2008; Verheijden et al., 2007). The suboptimal level of interactivity within the CT program might account for these findings. Interactivity has been defined as the degree of 2-way interaction that is provided in the program (Kiousis, 2002; Noar, Clark, Cole, \& Lustria, 2006), providing options for either immediate feedback or reciprocal interaction. Even though the program provided immediate feedback consisting of personalized health information, it did not provide communication features such as email contact and a discussion board, or features such as videos and games (Lustria, 2007). Effort should therefore be put into adjusting program components and content to the needs and wishes of this target group and into developing strategies to keep low-socioeconomic status participants engaged in the program.

\section{Sustained use}

Our results from the current study indicate that prompting visitors to revisit the CT program is an effective strategy to enhance sustained use. However, the effect was limited, with only small percentages of people actually responding to the prompt. Replicating this study is therefore desirable. A possible explanation for these results might be the level of tailoring in the prompt. Although the content of the prompt was tailored to user characteristics, the level of personalization may have been too low, making the prompt less appealing and relevant. Furthermore, since the first prompt was sent after 3 months, people may have forgotten about the program and their participation in it.

The added value of the email prompts used in the present study could be further augmented. Several studies have suggested that the effect of email prompts increases if they alert people to new content that is provided on the program website (Woodall et al., 2007) and if they provide information that is perceived as personally relevant (Fry \& Neff, 2009; Houston et al., 2010). Furthermore, the time of week when prompts are sent influences their effect, with prompts being sent at the beginning of the week being more effective (Houston et al., 2010).

Since email is a very popular online function used by substantial number of Internet users (The Radicati Group, 2011), it is a promising tool to attract people to healthpromoting interventions. However, a potential downside of its popularity is the frequent use of email for marketing purposes. Due to the increase in email advertise- 
ments, or spam, dissatisfaction among email users is increasing (The Radicati Group, 2011) and emails are often neglected or deleted before being read. Future studies should therefore aim at developing prompt content that attracts receivers' attention amidst a plethora of emails sent for marketing purposes. Furthermore, the optimal frequency at which prompts are delivered should be studied to yield further knowledge on future refinements and to maximize their potential.

\section{Strengths and limitations}

This study focused on exposure to an online CT lifestyle program by mapping characteristics of first-time users, examining predictors of first, prolonged and sustained use and by testing the efficacy of a proactive strategy to increase sustained use of the program. Even though several studies have highlighted the added value of periodic email prompts (Fry \& Neff, 2009), this study is one of the few to focus on the added value of periodic prompting by testing its effect in a randomized control trial.

However, this study is liable to several limitations that need to be accounted for when interpreting the results. First, we used one generic email to prompt revisits to the CT program. Future research should thoroughly study the optimal content of prompts, in order to make them stand out amidst advertisement emails and spam, and to make them more personally relevant and persuasive. Principles used in the field of e-marketing and e-commerce might provide essential information on specific strategies to increase the attractiveness of email prompts.

Finally, even though this study provides evidence for the effectiveness of prompts in enhancing program revisits, additional research is needed to study their effect on subsequent behaviour change because, due to a high dose-response relation, multiple visits to the program are necessary to maximize its effect on health behaviour.

\section{Conclusion}

We found that the CT modules were significantly more often initiated by older, male, employed, low-income participants with a relatively unhealthy lifestyle, which implies that the program succeeded in reaching and engaging those people who benefit most from online lifestyle interventions. However, people with a lower income tended to disengage from the CT program before finishing the modules. This underlines the importance of additional research into specific program adjustment and strategies that can be used to stimulate prolonged program use by low-socioeconomic status visitors. Furthermore, sending periodic email prompts significantly increased revisits to the program. Though promising, this effect was modest and needs to be further examined, in order to maximize the potential of periodic email prompting. 


\section{Chapter 6}

\section{Periodic email prompts to re-use an Internet-delivered lifestyle program: influence of prompt content and timing}




\section{ABSTRACT}

Adherence to Internet-delivered lifestyle interventions using multiple tailoring is suboptimal. Therefore, it is essential to invest in proactive strategies, such as periodic email prompts, to boost re-use. The objective of this study was to investigate the influence of content and timing of a single email prompt on re-use of an Internet-delivered lifestyle intervention. A sample of municipality employees was invited to participate in the program. All participants who decided to use the program received an email prompting them to revisit the program. A $2 \times 3$ (contentxtiming) design was used to test manipulations of prompt content and timing. Depending on the study group participants received either a prompt containing standard content (an invitation to revisit the program), or standard content plus a preview of new content placed on the program website. Participants received this prompt after 2,4 , or 6 weeks. In addition, a control condition was included, in which participants did not receive an additional email prompt. Clicks on the uniform resource locator (URL) provided in the prompt and log-ins to the CT program were objectively monitored. Logistic regression analyses were conducted to determine whether prompt content and/or prompt timing predicted clicking on the URL and logging in to the CT program. Of all program users ( $N=240), 206$ participants received a subsequent email prompting them to revisit the program. A total of 53 participants $(25.7 \%)$ who received a prompt reacted to this prompt by clicking on the URL, and 25 participants (12.1\%) actually logged in to the program. There was a main effect of prompt timing; participants receiving an email prompt 2 weeks after their first visit clicked on the URL significantly more often compared with participants that received the prompt after 4 weeks (OR 3.069, 95\% Cl 1.392-6.765, $\mathrm{P}=.005$ ) and after 6 weeks (OR 4.471, 95\% Cl 1.909-10.471, $\mathrm{P}=.001)$. Furthermore, participants who received an email prompt 2 weeks after their first visit logged in to the program significantly more often compared to participants receiving the prompt after 6 weeks (OR 16.356, 95\% Cl 2.071-129.196, $P=.008)$. A trend was observed with regard to prompt content. Participants receiving a prompt with additional content were more likely to log in to the program compared to participants who received a standard prompt. However, this result was not statistically significant (OR 2.286, 95\% Cl 0.892 $5.856, \mathrm{P}=.09$ ). The key findings suggest that boosting revisits to a $\mathrm{CT}$ program benefits most from relatively short prompt timing. Furthermore, a preview of new website content may be added to a standard prompt to further increase its effectiveness in persuading people to log in to the program. 


\section{INTRODUCTION}

Internet-delivered lifestyle interventions applying computertailoring techniques (Brug et al., 2005; Krebs et al., 2010; Ritterband et al., 2003) have reported positive effects for multiple health behaviours, such as physical activity (Van den Berg et al., 2007; Vandelanotte et al., 2007), fruit and vegetable intake (Brug et al., 1998; Neville et al., 2009), smoking cessation (Borland et al., 2004; Dijkstra, De Vries, \& Roijackers, 1998; Myung et al., 2009; Strecher, 1999) and alcohol consumption (Riper et al., 2008; White et al., 2010). Furthermore, providing computer tailored (CT) advice on multiple occasions (multiple tailoring) has proven to significantly add to their impact (Brug et al., 1998; Dijkstra, De Vries, Roijackers, \& van Breukelen, 1998). However, despite promising prospects, actual exposure to these interventions remains limited (Bennett \& Glasgow, 2009).

Exposure not only refers to the level of first-time use of the intervention, but also to the quality and quantity of intervention use (Crutzen, 2009; Schneider, van Osch, Schulz, et al., 2012). A small proportion of the potential target population actually accesses the intervention (Crutzen, 2009; Rogers, 1995; Schneider, van Osch, Schulz, et al., 2012), and the level of adherence to these interventions is even lower (Brouwer, Kroeze, et al., 2011), making attrition a common and urgent problem in Internet-based trials (Bennett \& Glasgow, 2009; Eysenbach, 2005). Non-usage attrition, in which participants lose interest in the intervention and refrain from continued use, is hindering actual impact on public health (Christensen et al., 2009; Eysenbach, 2005; Glasgow et al., 2011; Glasgow et al., 2007; Schneider, van Osch, Schulz, et al., 2012; Wanner, Martin-Diener, Bauer, Braun-Fahrlander, \& Martin, 2010).

Research on non-usage attrition has demonstrated an initial rapid decline in program use over the first few weeks (Bennett \& Glasgow, 2009; Eysenbach, 2005). During these weeks, participants lose interest in the program or realize that it does not meet their wishes or expectations. However, achieving health behaviour change is a 
complex and lengthy process requiring continuous guidance to maximize intervention effects (Christensen et al., 2009; Norman et al., 2007). Therefore, repeated use of interventions using multiple tailoring must be stimulated to allow processing of the entire intervention content and engagement in its effective components (Danaher et al., 2006; Eysenbach, 2005; Heesch, Masse, Dunn, Frankowski, \& Mullen, 2003; Strecher et al., 2008). Furthermore, re-use is important because it offers visitors the opportunity to self-monitor their level of behaviour change and receive additional personalized advice regarding strategies to increase or maintain their current level of behaviour change (Schneider, van Osch, \& de Vries, 2012). It is essential to minimize non-usage attrition at an early stage by investing in strategies that boost revisits to Internet-delivered interventions using multiple tailoring.

A substantial amount of Internet-delivered interventions use reactive strategies to achieve re-use, implying that a passive approach is used in which users themselves must undertake action to repeatedly benefit from the intervention content (Prochaska et al., 2001). However, because preventing non-usage attrition is a very strenuous process (Eysenbach, 2005; Glasgow et al., 2007; Verheijden et al., 2007), efforts should be put into attaining loyalty to the intervention directly after initiation by utilizing more proactive strategies (Schneider, van Osch, Schulz, et al., 2012). The use of periodic email prompts has been proposed as an effective proactive strategy to boost re-use of interventions (Fry \& Neff, 2009). Although the number of interventions employing proactive strategies is increasing, most studies merely explored the efficacy of the whole intervention, including prompting, instead of focusing on the added value of periodic prompting as a strategy to boost re-use. Furthermore, those studies indicating that sending periodic email prompts significantly increased program re-use (Fry \& Neff, 2009; Schneider, van Osch, Schulz, et al., 2012) recommended further examination and refinement to maximize their potential (Schneider, van Osch, Schulz, et al., 2012).

More specific evidence on the positive effects of the use of email to promote direct action stems from the field of e-marketing (Merisavo \& Raulas, 2004; Raad, Yeassen, Alam, Zaidan, \& Zidan, 2010). Within this field, the use of email advertisements is rapidly growing because of the increase in email users and their potential to reach large numbers of people at relatively low cost and effort. In addition, in the field of health promotion, emails have been used to prevent attrition in Web-based trials. Several studies have indicated that sending email reminders is an effective strategy to increase response rates in online data collection (Göritz \& Crutzen, 2012; Kongsved, Basnov, Holm-Christensen, \& Hjollund, 2007; Svensson, Svensson, Hansen, \& Trolle Lagerros, 2012). Furthermore, within one of our previous studies that examined the effect of using periodic email prompts (Schneider, van Osch, Schulz, et al., 2012), a 
positive, however modest, effect on re-use of the program was found. Within that study, an email prompt was sent 3 months after a first visit to the intervention. This modest effect might imply that a 3-month period is too long, causing people to forget about the program and their participation, which is in line with previous studies pointing out an elevated non-usage attrition level at the beginning of the intervention period (Bennett \& Glasgow, 2009; Eysenbach, 2005). Therefore, we recommended future research to focus on testing strategies to optimize the effect of email prompts (Schneider, van Osch, Schulz, et al., 2012). It is imperative to investigate the effect of using shorter prompting intervals and to determine the optimal interval at which prompts should be sent. In contrast, this modest effect of a periodic email prompt might also imply that prompt content is suboptimal. Since people tend to disengage from the intervention relatively shortly after intervention initiation because of a reduced levels of interest (Eysenbach, 2005), involvement in the intervention content and subsequent sessions is likely to decrease. According to the Elaboration Likelihood Model (ELM), people with a low level of involvement are less likely to process arguments used (Petty \& Cacioppo, 1986). As a result, argument-based persuasion techniques used in an email prompt persuading participants to re-use the intervention might be insufficiently processed. To increase persuasiveness of the email prompts for people with a low level of involvement in the issue, the current study tested the effect of adding a peripheral cue to a standard email prompt (Petty \& Cacioppo, 1986). This peripheral cue consisted of the addition of a preview of new website content. This preview served as a teaser to increase curiosity for the remaining website content (Menon \& Soman, 2002). Instead of basing a decision to re-use the program on the argument posed in the email, participants might simply react out of sheer curiosity (Petty \& Cacioppo, 1986). It was hypothesized that a prompt with additional content was more effective in persuading people to revisit the CT lifestyle program compared to a standard prompt.

\section{METHODS}

This study aimed to assess the added value of periodic email prompts to boost revisits to an Internet-delivered CT lifestyle program. We aimed to answer 2 questions: (1) which prompt timing interval is most effective in boosting re-use of the Internetdelivered CT lifestyle pro-gram and (2) which prompt content is most effective in boosting re-use of the program? We addressed these questions among participants in an Internet-delivered CT intervention aimed at multiple health behaviours: increasing physical activity, increasing fruit and vegetable intake, smoking cessation, and decreasing alcohol consumption. 


\section{Procedure and participants}

This study was conducted in close collaboration with the Regional Public Health Service (RPHS) in the northern part of the Dutch region, Limburg. All people employed by the municipalities in this region were invited by the RPHS to participate in an Internetdelivered CT lifestyle program developed by our research group (Schneider et al., 2011; Schulz et al., 2011). Invitations for this program were placed on the intranet of all municipalities and were also directly emailed to employees. The invitation contained a uniform resource locator (URL) that directed interested participants directly to the program. The program provided employees the opportunity to receive free-ofcharge CT feedback about their current health behaviour (physical activity, fruit and vegetable intake, alcohol consumption, and smoking) and assistance in changing these health behaviours.

Participants who logged in to the program to obtain CT advice were sent an email prompting them to re-use the program. Re-use was encouraged to allow participants to monitor their behaviour change and to obtain iterative CT advice aimed at behaviour change and relapse prevention. Furthermore, participants were offered an opportunity to obtain CT advice regarding an additional health behaviour. Finally, re-use was stimulated to keep participants informed about new content added to the program website.

\section{Design}

A $2 \times 3$ design was used to test the effect of 2 factors: prompt content (standard and standard + ) and prompt timing (2, 4, and 6 weeks). The standard prompt (SP) contained a message reminding people about their previous visit to the program and invited them to re-use the program to monitor their progress and obtain additional feedback. The second version of the prompt (SP+) contained standard content complemented with a message alerting people to new content added to the intervention website. Participants received an email prompt after 2, 4, or 6 weeks. In addition to these 6 experimental conditions, a control condition was included, no prompt (NP). People allocated to the control condition did not receive an additional prompt and were only encouraged at baseline to re-use the program. To participate in the program, participants had to register by using a personal log-in code and password. Immediately after registration for the program, participants were randomly allocated to 1 of the 7 study conditions. Randomization occurred at the respondent level by means of a computer software randomization device. Data for the present study were collected from March to July 2011. 


\section{Email prompt}

People who were allocated to the experimental conditions of the study received an email prompting them to re-use the program. Periodic prompts should be distinguished from re-minders, which are also often used in Internet-delivered interventions. Sending reminders is a technique that is used to increase response rates in online data collection by proactively stimulating participation among non-responders and is used to prevent drop-out attrition (Eysenbach, 2005; Göritz \& Crutzen, 2012). Periodic prompts, on the other hand, are used to boost re-use of the intervention content by approaching all participants and are used to prevent non-usage attrition (Eysenbach, 2005). All email prompts used in the present study contained standard content. This standard email opened with a personalized greeting and reminded people about their first visit to the pro-gram. Subsequently, people were invited to re-use the program to obtain information about their current health status and to monitor their progress. Participants were also given the opportunity to receive additional iterative health advice on the health behaviour(s) selected at baseline or on a new behaviour (Appendix 3). Finally, to facilitate logging in to the program, the email also contained details about their personal log-in information (username and password). The email concluded with greetings from the research team and contact information. Half of all people in the experimental conditions received an email that also contained additional content (SP+). This additional content consisted of a preview of new information that was placed on the program website since they last visited it (Appendix 4). This information referred to nutrition and provided examples of healthy food alternatives that were available for that current season (e.g. spring/summer).

\section{Internet-delivered computer tailored lifestyle intervention}

The CT program integrated established CT programs tested and proven to be effective in randomized controlled trials for increasing smoking cessation, promoting the intake of fruit and vegetables, increasing the level of physical activity, and reducing the consumption of alcohol (Poel et al., 2009; Smeets et al., 2007; Smit, de Vries, \& Hoving, 2010; Van Keulen et al., 2008). The program used a dual approach to guide people toward behaviour change. First, awareness of participants' current health behaviour status was increased by comparing their status to the Dutch public health guidelines set for these health behaviours, such as being moderately physically active for 30 minutes at least 5 days a week, eating 2 pieces of fruit per day, eating 200 grams of vegetables per day, not drinking more than 1 (women) or 2 (men) glasses of alcohol a day, and not smoking. Second, assistance was provided in changing participants' health behaviour by using CT modules available per behaviour. The modules used a fixed, gradual approach consisting of four steps, guiding people toward behaviour 
change based on the Integrated Model for exploring motivational and behavioural change (I-Change Model) (De Vries et al., 2003). Focus was on pros and cons of the desired behaviour (step 1), the role of significant persons in the direct environment (step 2), preparatory plans assisting people to start changing their behaviour (step 3), and coping plans to help them overcome difficult situations and prevent relapse (step 4). Within the modules, all health advice was adapted to the individual's characteristics by considering demographic, behavioural, and cognitive characteristics (De Vries \& Brug, 1999; Dijkstra \& De Vries, 1999; Kreuter et al., 2000). The Internet-delivered CT lifestyle program is described in more detail elsewhere (Schneider et al., 2011; Schulz et al., 2011).

The program was embedded in a website designed for the current study. This website entailed general information considering a healthy lifestyle and the selected health behaviours. Furthermore, the website provided specific information regarding the project, contained a direct link to the CT program, and provided background information regarding the study and the research team. During the study, new information (e.g. advice, supporting messages, recipes, and facts) was added to the website.

\section{Measures}

Participants in the experimental conditions (SP and SP+) received an email prompting them to re-use the intervention. Participants had to take 2 steps to re-use the program in order to self-monitor their level of behaviour change and obtain additional CT advice. The first was clicking on the URL provided in the email prompt. Clicking on the URL was tracked by referral ID codes that were integrated in the URL. The second step was logging in to the CT program after arriving on the program website. Log-ins were objectively monitored during a 2 - week period and log-in dates were compared to baseline dates to determine revisits.

To describe characteristics of program visitors, age, gender, and educational level of participants was assessed (1/low: no education or lower vocational school; 2/medium: secondary vocational school or high school; 3/high: higher professional education or university). Health behaviour status consisted of information regarding the 5 key behaviours. Physical activity was measured by the Short Questionnaire to Assess Health-enhancing physical activity (SQUASH) (Wendel Vos et al., 2003) and guideline adherence was calculated following procedures used by Ainsworth (Ainsworth et al., 2000). Fruit consumption was measured by using a 4-item Food Frequency Questionnaire (FFQ) assessing weekly amount of fruit and fruit juice intake (RIVM, 2005), whereas vegetable consumption was measured using a 4-item FFQ assessing the weekly amount of consumed boiled or baked vegetables as well as salad 
or raw vegetables (RIVM, 2005). The consumption of alcohol was measured by the Dutch Quantity-Frequency-Variability (QFV) questionnaire (Lemmens et al., 1992). Finally, smoking status was assessed by asking participants whether they smoked, what they smoked (cigarettes, cigars, pipe tobacco), and how much they smoked per day (cigarettes) and per week (cigars/packets pipe tobacco) (De Vries, Kremers, et al., 2008). For each health behaviour, a new variable was created to indicate whether participants met the Dutch guidelines provided for these behaviours ( $0=$ no; $1=y e s$ ).

\section{Statistical analyses}

General descriptive statistics were calculated to describe characteristics of program visitors, as well as main findings concerning adherence to the public health guidelines. Baseline differences between the intervention groups were calculated using the Chisquare test for dichotomous and categorical variables and 1-way analysis of variance (ANOVA) for continuous variables. Finally, logistic regression analyses were conducted. There were 2 dependent variables: whether participants clicked on the URL $(0=$ no; $1=y e s)$ and whether participants logged in to the CT program ( $0=$ no; $1=y e s)$. Prompt content (dummy coded, $\mathrm{SP}=0 ; \mathrm{SP}+=1$ ) and prompt timing (dummy coded with 2 weeks as the reference category and 4 weeks as the reference category) and the interactions between these variables were used as predictors in the initial model of each dependent variable. Furthermore, because women and people with a higher educational level and age are more likely to use Internet-delivered lifestyle interventions (Schneider, van Osch, Schulz, et al., 2012; Brouwer et al., 2010) age, gender, and educational level were included in the models as possible covariates. An alpha of .05 was used to indicate statistical significance. All statistical analyses were done with the program SPSS 17.0 (SPSS Inc, Chicago, IL, USA).

Table 6.1. Participants who clicked on the URL (step 1) and logged in to the program (step 2) per study condition $(\mathrm{N}=\mathbf{2 4 0})$

\begin{tabular}{|c|c|c|c|c|c|c|c|}
\hline \multirow[t]{3}{*}{ Dependent variable } & \multicolumn{7}{|c|}{ Condition, n (\%) } \\
\hline & \multicolumn{3}{|c|}{ Standard content } & \multicolumn{3}{|c|}{ Standard+ content } & \multirow{2}{*}{$\begin{array}{l}\text { No } \\
\text { prompt } \\
n=34\end{array}$} \\
\hline & $\begin{array}{l}2 \text { weeks } \\
n=34\end{array}$ & $\begin{array}{l}4 \text { weeks } \\
n=34\end{array}$ & $\begin{array}{l}6 \text { weeks } \\
n=35\end{array}$ & $\begin{array}{l}2 \text { weeks } \\
n=36\end{array}$ & $\begin{array}{l}4 \text { weeks } \\
n=35\end{array}$ & $\begin{array}{l}6 \text { weeks } \\
n=32\end{array}$ & \\
\hline Click on the URL & $14(41.2)$ & $6(17.6)$ & $4(11.4)$ & $16(44.4)$ & $7(20.0)$ & $6(18.8)$ & - \\
\hline Log in to the program & $6(17.6)$ & $1(2.9)$ & $1(2.9)$ & $10(27.8)$ & $7(20.0)$ & $0(0.0)$ & $2(5.9)$ \\
\hline
\end{tabular}




\section{RESULTS}

In total, 240 participants visited the program, of which 73.3\% (176/240) were female. Participants were randomly allocated to each of the 7 study conditions and a randomization check revealed that females were equally distributed ( $\chi 26=9.1, p=.17$ ). Furthermore, there were no significant differences between the groups regarding educational level $(\chi 212=9.3, p=.68)$ and age $(F 6,233=0.464, p=.84)$. Overall visitors had a mean age of 50 years (SD 14.99) and most were medium (100/240, 41.7\%) to highly educated (111/240, 46.3\%). Regarding the 5 health behaviours included in the program, $12.9 \%$ (31/240) did not comply with the Dutch guidelines of at least 30 minutes of moderately intensive physical activity on at least 5 days of the week. With regard to fruit and vegetable intake, 50.8\% (122/240) and 60.0\% (144/240) did not adhere to the Dutch guidelines of at least 2 pieces of fruit and at least 200 gram of vegetables each day, respectively. Approximately 1 out of 10 participants indicated that they smoked (10.0\%, 24/240), and 22.1\% (53/240) did not comply with the Dutch guidelines for alcohol intake.

A total of 206 participants received an email prompting them to re-use the program. Of this sample, 53 participants $(25.7 \%)$ reacted to this email by clicking on the URL (step 1), whereas 25 participants (12.1\%) actually logged in to the program (step 2 ). All results concerning clicking on the URL and logging in to the program are described per study group in Table 6.1.

Table 6.2. Effects of prompt content and timing on clicking on the URL and logging in to the program

\begin{tabular}{llll}
\hline Dependent variable & $\mathrm{OR}^{a}$ & $\mathrm{p}$ & \\
\cline { 2 - 3 } & & & \\
Clicking on the URL (1) & & \\
Prompt content (SP versus SP+) & & \\
Prompt timing (2W versus 4W) & 1.28 & .48 & $0.65-2.51$ \\
Prompt timing (2W versus 6W) & 3.07 & $.00^{*}$ & $1.39-6.77$ \\
Prompt timing (4W versus 6W) & 4.47 & $.00^{*}$ & $1.91-10.47$ \\
& 1.46 & .43 & $0.58-3.69$ \\
Logging in to the program (2) & & & \\
Prompt content (SP versus SP+) & & .09 & $0.89-5.86$ \\
Prompt timing (2W versus 4W) & 2.29 & .12 & $0.82-5.59$ \\
Prompt timing (2W versus 6W) & 2.14 & $.00 *$ & $2.07-129.20$ \\
Prompt timing (4W versus 6W) & 16.36 & .06 & $0.02-1.02$ \\
\hline
\end{tabular}

${ }^{a}$ Odds ration; ${ }^{b}$ Confidence interval.

* $p<.05$ 


\section{Step 1 - clicking on the URL}

There was no significant interaction between prompt content and prompt timing (Figure 6.1) with regard to clicking on the URL. Therefore, interaction terms were excluded from the remaining models and only main effects are reported.

Analyses of main effects indicated that there was a significant effect of prompt timing ( $x 22=15.2, p<.001)$. Participants who received an email prompt 2 weeks after their first visit, clicked on the URL significantly more often compared with participants that received the prompt after 4 weeks (odds ratio [OR] 3.069, 95\% Cl 1.392-6.765, $\mathrm{P}=.005$ ) and after 6 weeks (OR 4.471, 95\% Cl 1.909-10.471, $p=.001$ ). There was no significant difference in reaction to the email prompt between participants receiving the prompts after 4 weeks, compared with participants receiving the prompts after 6 weeks. Also, no main effects of prompt content could be detected (see Table 6.2).

Figure 6.1. Percentages of participants given standard prompt (SP) and standard+ prompt (SP+) who clicked on the URL at different levels of prompt timing

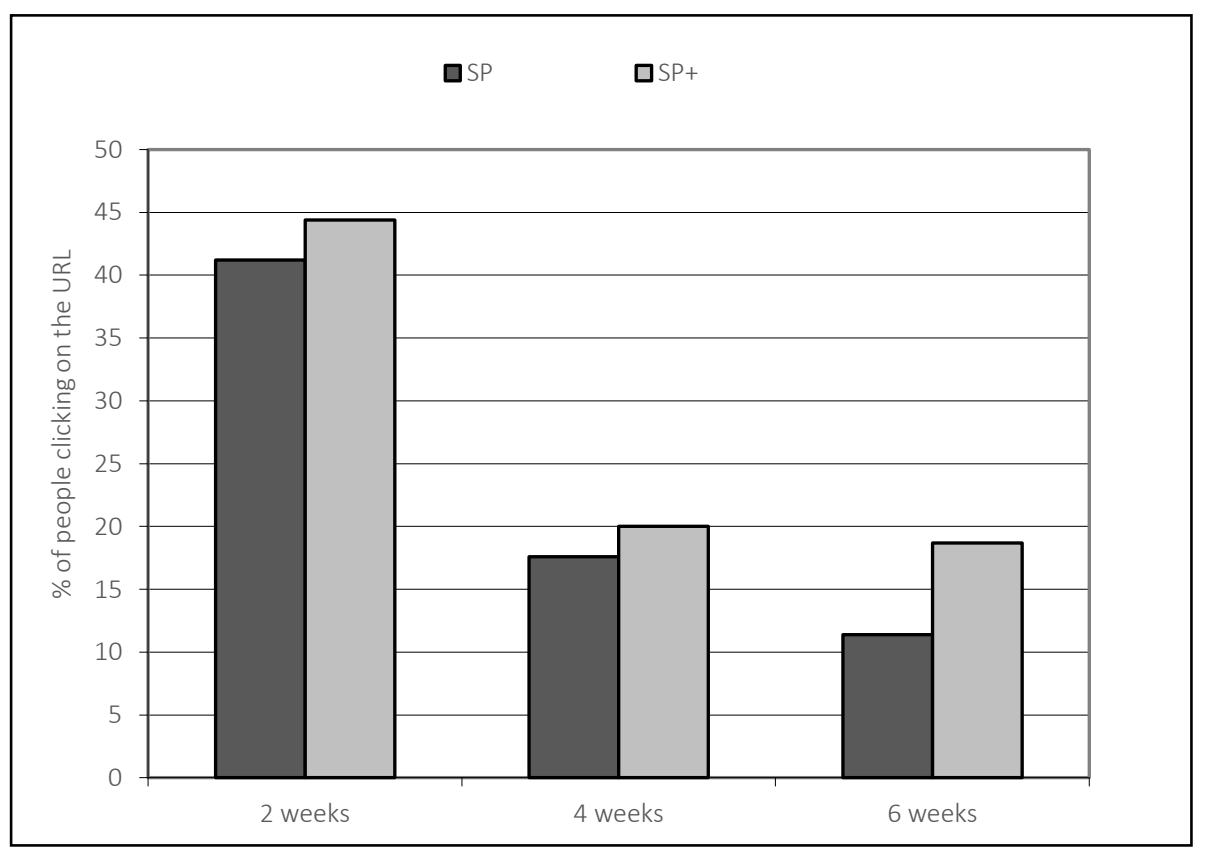

Step 2 - logging in to the program

With regard to logging in to the program, no significant interaction between prompt 
content and prompt timing was found (Figure 6.2). Therefore, interaction terms were again excluded from the remaining models and only main effects are reported.

With regard to logging in to the program, analyses of main effects indicated that there was a significant effect of prompt timing $(\chi 22=16.5, p<.001)$. Participants receiving an email prompt 2 weeks after their first visit, logged in to the program significantly more often compared to participants receiving the prompt after 6 weeks (OR 16.356, 95\% Cl 2.071-129.196, $p=.008)$. There was, however, no significant difference between participants receiving the prompts after 4 weeks compared with participants receiving the prompts after 2 or 6 weeks. With regard to prompt content, a trend was observed. Participants receiving a prompt with additional content were more likely to log in to the program compared to participants who received a prompt with standard content. However, this result was not statistically significant (OR 2.286, 95\% Cl 0.892$5.856, p=.09)$.

Figure 6.2. Percentages of participants given standard prompt (SP) and standard+ prompt $(S P+)$ who logged on to the intervention at different levels of prompt timing

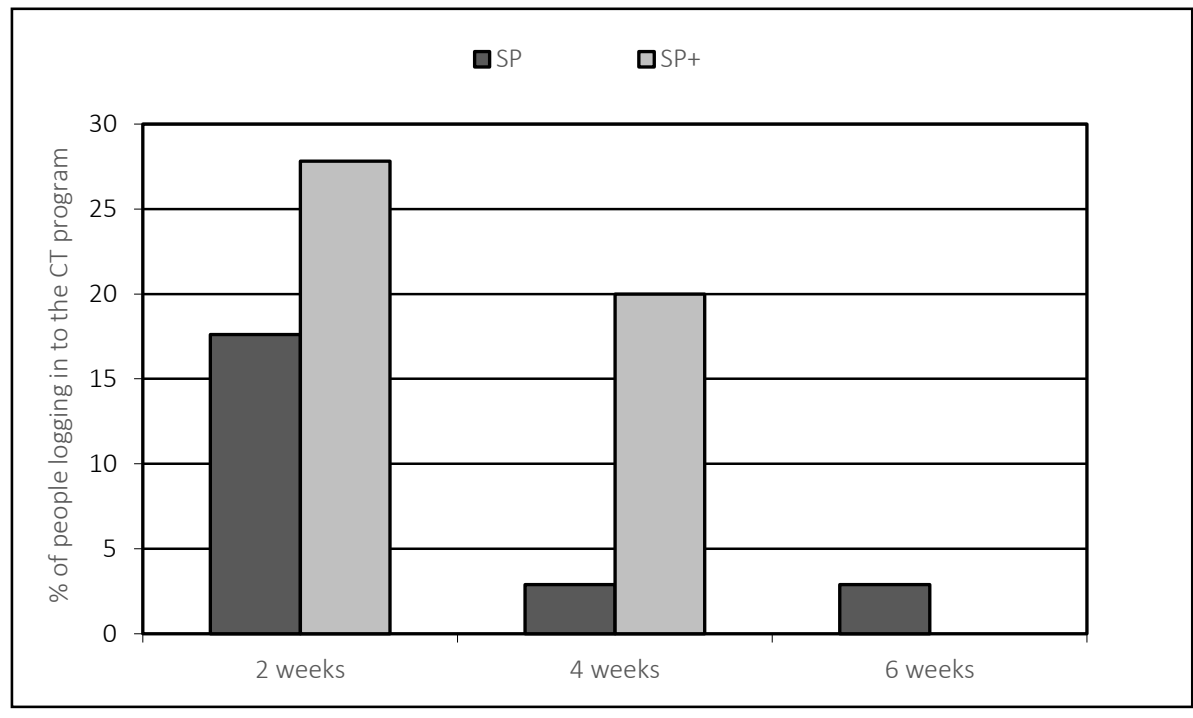

Finally, all experimental groups were compared with the control condition (NP) that did not receive an additional email prompt to boost revisits to the intervention. Results from this analysis revealed that participants who received an email prompt containing additional con-tent after 2 weeks were significantly more likely to log in to the 
program (OR 6.059, 95\% Cl 1.195-30.726, $p<.001$ ).

\section{DISCUSSION}

This study aimed at maximizing the potential of email prompts by focusing on prompt content and timing. A single email prompt was used in the context of an Internetdelivered CT lifestyle program and was aimed at stimulating re-use to increase the dose of the intervention and prevent non-usage attrition. Firstly, results indicated that sending a prompt 2 weeks after the first visit was more effective compared with using a longer time period. This effect not only referred to clicking on the URL (step 1), but also to the proportion of log-ins to the program (step 2). Secondly, a trend was observed indicating that adding a preview of new website content to a standard prompt increased its effectiveness in persuading people to log in to the program. Finally, sending a prompt with additional content after a 2-week period significantly increased program log-ins compared to using a reactive approach in which no additional prompts were used.

The effectiveness of using a relatively brief interval for sending a prompt may indicate that people tend to forget about the program. Therefore, the interval between engaging in a first visit and receiving an email prompt to boost re-use should be kept relatively short. Since the costs of sending a large number of email messages are relatively low (Merisavo \& Raulas, 2004), this strategy allows for sending several emails at relatively short intervals. However, when using an email prompt to boost reuse of the program, one must keep the goal of the revisit in mind. Within the current program, the prompt was used to remind people about their previous visit to the program and to persuade them to re-use the program to monitor their progress and obtain additional feedback. Although results from this study suggest that prompt timing should be short, one must allow participants enough time to actually follow the obtained advice and develop strategies to positively change their lifestyle (Verheijden et al., 2007).

Results from the current study also show a trend toward the efficacy of adding additional content to a standard prompt. However, this trend was only present for logging in to the program (step 2) and not for clicking on the URL (step 1). A possible explanation for this effect on log-ins might be the repeated exposure to new website content. People who received an email prompt containing a preview of new website content were exposed to this website content twice: within the email prompt and on the program website. According to ease of processing theory (Whittlesea, Jacoby, \& Girardi, 1990), repeated exposure to information allows for more fluent processing of the information. This extended degree of exposure might have enhanced the ease of processing the new information added to the website. Because this new information 
was related to health and emphasized the importance of having a healthy lifestyle, this information could have persuaded people to monitor their current lifestyle behaviour and obtain additional advice about how to positively change their behaviour.

With regard to clicking on the URL (step 1 ), significant effects of adding a peripheral cue to a standard prompt failed to occur. People were significantly more likely to respond to emails that were sent at a relatively short interval, irrespective of the email content. A possible explanation for this finding might be that participants consented to participate in the present study after being informed about the possibility of receiving an additional email to boost program use. Within the field of e-marketing, the term opt-in email is used when receivers have agreed to the receipt of an email (Barnes \& Scornavacca, 2004; Raad et al., 2010). Research has indicated that opt-in emails are largely accepted among receivers and are proven to be more effective compared to spam email (receiver has not agreed to receiving an email) when used to persuade people to undertake action (e.g., clicking on a URL to re-use the program) (Barnes \& Scornavacca, 2004; Raad et al., 2010). Therefore, knowledge about receiving an email prompt might have increased acceptance of the email among participants and might have resulted in the desired behaviour (clicking on the URL) irrespective of the email content.

\section{Strengths, limitations and recommendations}

The current study primarily focused on the effectiveness of using a single email prompt with regard to clicking on the URL and logging in to the program. Combined with results from our previous study (Schneider, van Osch, Schulz, et al., 2012), important information regarding the overall effectiveness of using a single email prompt as well as the optimal prompt content and timing was obtained. To our knowledge, this is one of the first studies that systematically studied optimal content and timing of a single email prompt used to boost re-use of a multiple CT lifestyle program offered through the Internet. Although these results suggest that sending a single email prompt is an effective strategy to boost revisits to interventions using multiple computer-tailoring, this is only a first step, and the findings should be interpreted keeping several limitations in mind. Firstly, the current study focused solely on sending an email prompt on 1 occasion, namely after 2, 4, or 6 weeks. However, achieving health behaviour change is a complex and enduring process that requires continuous guidance (Christensen et al., 2009; Norman et al., 2007). Repeated visits to interventions using multiple computer-tailoring are imperative to ensure notification of the entire intervention content and involvement in its effective components (Danaher et al., 2006; Strecher et al., 2008) and must, therefore, be encouraged. Additional research should go one step further and focus on timing intervals and prompt frequency. 
Prompting for revisits shortly after a first visit indeed stimulated revisits to the program. However, repeatedly sending prompts at a certain interval to boost multiple revisits might have different or even reverse effects because responses to multiple prompts tend to gradually decline over time (Woodall et al., 2007). Secondly, effectiveness of the use of an email prompt largely depends on the degree to which the content is actually read and processed by receivers. Within the current study, no objective measure of the degree to which emails were read was obtained. Therefore, results on prompt content should be interpreted with caution. Future research should put additional effort in assessing the degree to which email content is actually read and processed by participants. Furthermore, additional research is needed to investigate the effects of using periodic prompts on behaviour change and to investigate whether these effects remain present over a sustained period of time. Thirdly, the sample size for the present study was relatively small. As a result, the study might be relatively underpowered to study interaction effects and to indicate significant main effects. Furthermore, regression analyses resulted in several substantial confidence intervals. Although these results should be interpreted with caution, they still provide valuable information regarding the effectiveness of timing and content of a single email prompt as a strategy to boost program revisits. Fourthly, a related limitation is the representativeness of the study sample. This sample does not provide a good cross-section of the general Dutch population (Centraal Bureau voor de Statistiek, 2011) because participants were mainly female, middle-aged, and medium to highly educated. This may limit possibilities for generalizing our results to the general population. However, previous studies repeatedly indicated that Internet-delivered CT programs tend to predominantly reach women and people who are older and higher educated (Brouwer et al., 2010; Schneider, van Osch, Schulz, et al., 2012; Schulz et al., 2012; Spittaels \& De Bourdeaudhuij, 2007; Spittaels, De Bourdeaudhuij, Brug, et al., 2007; Vandelanotte et al., 2005). As a consequence, the current sample corresponds to the subgroup of people known to be reached by Internet-delivered CT programs, although it does not represent the general population. Therefore, the obtained results are valuable in the context of CT lifestyle programs that are offered through the Internet.

\section{Conclusions}

We found that using relatively short prompt timing ( 2 weeks) resulted in more positive effects compared to using a longer time period. This effect not only referred to clicking on the $U R L$, but also to the proportion of log-ins to the program. Although a trend was observed about the effectiveness of adding a preview of new website content to a standard prompt, this only referred to persuading people to log in to the 
program. The findings of this study underline the importance of sending an email prompt relatively shortly after a first visit to the program. Furthermore, it is important to further focus on prompt content because the addition of a peripheral cue may add to the prompt's effectiveness. 


\section{Chapter 7}

General discussion 

The main aim of this thesis was to gain more insight into reach and use of the Internet-delivered lifestyle intervention. In accordance with the RE-AIM framework reach was defined as the absolute number, proportion and representativeness of potential users who were willing to participate in the intervention (Glasgow et al., 1999). Intervention use was subdivided into actual adoption of the intervention (first use), extending a first visit by engaging in the intervention session (prolonged use) and revisits to the intervention (sustained use) (Crutzen, 2009). This final chapter describes the main findings of the studies presented in this thesis. It provides a summary of the main results categorized in first, prolonged and sustained use and relates the obtained results to previously conducted studies. Furthermore, it considers several important methodological and practical considerations that should be kept in mind while interpreting the main results. Finally, this chapter addresses recommendations of the main findings for both research and practice.

\section{MAIN FINDINGS RELATED TO FIRST USE}

In order to optimize reach of the Internet-delivered lifestyle intervention a proactive dissemination strategy was used. This proactive strategy consisted of embedding the program in the Adult Health Monitor of the Regional Public Health Services in the Dutch provinces of North-Brabant and Zeeland. Success of this strategy was determined by studying the absolute number, as well as proportions of program adopters. Next to this, adequacy of reach was determined by studying user characteristics that predicted interest and participation in the Internet-delivered lifestyle intervention. 


\section{Success of the proactive dissemination strategy}

In total, 41.155 invitees participated in the Adult Health Monitor of 2009, of which $41 \%$ completed the online version of the Adult Health Monitor. Since the Internetdelivered intervention was only offered to online Adult Health Monitor participants, the absolute number of participants that could have been proactively reached with the Internet-delivered lifestyle program was 16.940. In absolute numbers, 9.169 participants indicated their interest in the program, 54\% of all online Adult Health Monitor participants. 5.168 participants actually paid a first visit to the Internet-delivered lifestyle intervention. This means that $31 \%$ of all online Adult Health Monitor participants and $56 \%$ of all interested participants visited the intervention (chapter 3 ).

Since the intervention was offered proactively within the controlled environment of the Adult Health Monitor to a large representative sample of Dutch inhabitants, exact numbers and proportions of reach could be determined (Glasgow et al., 1999). In order to accurately assess reach of an employed recruitment strategy, it is important to describe the amount of people that are exposed to the recruitment strategy, the number of people that respond to this recruitment strategy and the number of people that participate in the intervention (Graham, Bock, Cobb, Niaura, \& Abrams, 2006).

Within the current study, we were able to obtain accurate figures of people exposed to the proactive dissemination strategy (i.e. online Adult Health Monitor participants), people who responded to this strategy (i.e. people that indicated their interest in the intervention) and actual intervention participants (i.e. people that paid a first visit to the intervention). These collected figures are fairly exceptional, since in most Internet trials, these numbers are often difficult or impossible to retrieve, making it difficult to apply precise denominators to establish the proportion of participants reached (Dzewaltowski, Glasgow, Klesges, Estabrooks, \& Brock, 2004). Furthermore, recruitment strategies for Internet-delivered interventions can be very versatile, ranging from mass media approaches, to recruitment via intermediaries like general practitioners, family or friends (Brouwer, Oenema, et al., 2011; Crutzen et al., 2009a; Smit, Hoving, Cox, \& de Vries, 2012). Inaccurate estimates of reach, combined with divergent recruitment strategies make it challenging to compare reach of the current strategy to other strategies and studies. In addition, the Internet-delivered lifestyle intervention was proactively disseminated in a real life setting, whereas the majority of interventions are disseminated in a trial setting, making the number of studies available for comparison even lower. Estimates of reach of the current intervention were higher compared to a similar Internet-delivered lifestyle intervention that was offered to a comparable sample in a real-life setting, which reported approximate participation rates of 3\% (Brouwer et al., 2010). Previous research has indicated 
that it can be expected that reach of these interventions may be even less optimal when implemented in a real life setting (Evers et al., 2005; Evers et al., 2003). Results from efficacy trials outside real life settings, have demonstrated participation rates ranging from 2.5\% to 20\% (Brouwer et al., 2010; Glasgow et al., 2007; Leslie et al., 2005). When comparing the employed dissemination strategy to participation rates in efficacy trials, the strategy can be considered successful, as we managed to obtain participation rates of $31 \%$. This percentage is calculated by using the total online sample of the Adult Health Monitor ( $n=16.940)$. Even when using a more conservative estimate of reach, by determining this rate using all Adult Health Monitor participants as the denominator, including people that did not complete the online version $(n=41.155)$, we obtained a participation rate of $13 \%$. Even though substantially smaller, this percentage is still in the higher spheres considering reported and available percentages of reach of Internet-delivered interventions. Therefore, based on these findings, we conclude that our proactive dissemination strategy was successful in optimizing reach of the Internet-delivered computer tailored lifestyle intervention, as one out of three invited and eligible participants decided to pay a first visit to the intervention.

\section{Adequacy of reach of the proactive dissemination strategy}

With regard to adequacy of reach, results indicated that online Adult Health Monitor participants were more likely to be younger, male and relatively highly educated compared to participants that used the written version of the Adult Health Monitor. Furthermore, being employed and in a relationship, as well as having a relatively unhealthy lifestyle were identified as important predictors of online Adult Health Monitor participation. Taking a closer look at this online sample, results revealed that participants indicating their interest in the Internet-delivered intervention were predominantly older, male, and relatively highly educated compared to people that were uninterested. In addition, these participants were more often unemployed, not in a relationship, born in the Netherlands and these participants suffered from relatively more symptoms of depression and anxiety. Finally, first use of the intervention among interested participants was predicted by gender, educational level and lifestyle status; program users were inclined to be male, older and higher educated, with a healthier lifestyle and a lower BMI compared to non-users (chapter 3 ).

By embedding the Internet-delivered lifestyle intervention in the Adult Health Monitor environment, we yielded insight into characteristics of program users, as well as of people that refrained from using the program. This allowed us to focus on adequacy of reach and to compose detailed profiles of program users and non-users. Even though the proportion of people that was reached was relatively high, results 
indicated that program participation was modest in specific at-risk subgroups. Firstly, results indicated that relatively higher educated participants were more likely to participate in the Internet-delivered intervention. This finding is in line with previous studies examining use of Internet-delivered lifestyle interventions, where the majority of participants had a relatively high educational level (Brouwer et al., 2010; Lorence \& Park, 2008; Spittaels \& De Bourdeaudhuij, 2007; Yang et al., 2008). Secondly, intervention participants reported a relatively healthier lifestyle and a healthy BMI. These results are also to a large extent in line with previous findings concerning use of Internet-delivered computer tailored programs (Brouwer et al., 2010; Spittaels \& De Bourdeaudhuij, 2007; Strecher et al., 2008; Verheijden et al., 2007). Since people with an unhealthy lifestyle may benefit more from (Internet-delivered) lifestyle interventions compared to people with a healthy lifestyle, it is essential to increase reach of Internet-delivered interventions among these subgroups and further investigate and optimize suitable dissemination strategies. Previous research has, for instance, indicated that recruitment for an Internet delivered smoking cessation intervention via general practitioners resulted in a higher proportion of participants with a lower educational level and an unhealthier lifestyle compared to recruitment via mass-media. However, it is important to mention that even though recruitment via general practitioners resulted in higher proportions of at-risk participants, a mass-media recruitment strategy resulted in elevated absolute number of at-risk participants (Smit et al., 2012). Similar results were found in another study that compared recruitment for an Internet-delivered lifestyle program via general practitioners to recruitment via a flyer (Brouwer, Oenema, et al., 2011). Results indicated that recruitment via general practitioners resulted in a higher percentage of intervention users, whereas a flyer resulted in higher absolute numbers of intervention users, making this strategy more costeffective (Brouwer, Oenema, et al., 2011) In addition, recruitment via general practitioners was more successful in reaching at-risk participants, as the majority of intervention users had a lower educational level and a higher BMI. This indicates that the selected strategy may not only have an effect on the proportion of people that is reached, but also on the 'type' of participants that are recruited.

\section{MAIN FINDINGS RELATED TO PROLONGED USE}

The second aim of the current project was to optimize prolonged use of an Internetdelivered lifestyle intervention by paying more attention to this aspect during intervention development. The intervention was an evidence-based Internet-delivered computer tailored lifestyle intervention, which integrated content from evidencebased interventions aimed at increasing smoking cessation, promoting the intake of 
fruit and vegetables, increasing the level of physical activity and reducing the consumption of alcohol (Brug et al., 1999; Poel et al., 2009; Smeets et al., 2008; Van Keulen et al., 2011). Results from a Delphi study on factors related to optimal development of health-related websites, combined with results from focus groups and a process evaluation, guided adjustment of the intervention content as well as the environment in which the intervention was embedded by incorporating the needs and wishes of prospective users and experts from the fields of health promotion, health psychology, e-communication and web design. Prolonged use of the intervention was closely monitored by studying levels of module initiation and completion and by studying predictors of initiation and completion.

\section{Delphi study on intervention development}

Development of the Internet-delivered computer tailored lifestyle intervention content and its online environment was guided by results from the Delphi study (step 1). This resulted in a first version of the intervention. This version was further refined by conducting focus groups interviews with potential futures users $(N=20)$ using a thinkaloud approach (step 2). These focus group interviews resulted in valuable insights with regard to experiences of users and their opinions about intervention content, appeal and ease of use. These insights were further complemented by results from a process-evaluation ( $\mathrm{N}=521$ ), which provided valuable information with regard to relevance of the intervention content, ease of use and satisfaction with the intervention content (step 3). Only results from the first step, entailing the Delphi study, are discussed in this thesis.

By means of a Delphi study factors related to optimal development of healthrelated websites were identified and agreed upon by experts and potential users. This resulted in an extensive list of factors that potentially contributed to optimizing the development of health-related websites. These factors should therefore be addressed when developing the Internet-delivered lifestyle intervention. The results of the Delphi study are largely in line with previous studies. Furthermore, the factors identified in this Delphi study are in concordance with the Model of Internet Intervention (Ritterband et al., 2009), which proposes several intervention factors to have an influence on intervention use and effect. These factors range from appearance of the website, to behavioural prescription, the size of the burden that actual use of the website entails, content of the intervention, the mode of delivery, the intervention message(s), participation and assessment. With regard to appearance of the website, experts and users suggested the importance of appealing websites, with a professional appearance. Aesthetics of websites, or their appearance are detected first and these may influence users' behaviour on the website (Lindgaard, Fernandes, Dudek, \& 
Brown, 2006). Server statistics of an Internet-delivered computer tailored lifestyle intervention implemented for use by the general public revealed that the majority of the visitors (56.3\%) left the intervention website within 30 seconds (Brouwer et al., 2010). Evaluations of a website are often based on first impression, hereby spending only limited amounts of time on actual interaction with the website (Lindgaard et al., 2006). It is therefore important to ensure an attractive appearance of the website. Furthermore, both experts and potential users stressed the importance of providing a behavioural prescription that entails information on how to obtain a healthy lifestyle. Another factor identified in this Delphi study referred to the size of the burden that actual use of the website entails. Both experts and potential users stressed the importance of an easy log-in procedure, a clearly structured website and a simple website design, which is in line with results from previous studies (Brouwer et al., 2008; Eysenbach \& Kohler, 2002). Furthermore, with respect to optimizing questionnaire completion, a recent study also recommended the use of a progress bar and an option for partial completion (Crutzen, 2010). Findings of a recent meta-analysis further extended these recommendations by indicating that progress bars that decelerate during questionnaire completion are more effective in reducing drop-out compared to bars that speed up during completion (Villar, Callegro, \& Yang, 2013). The provision of personalized (or tailored) feedback regarding the status of lifestyle behaviours and the opportunity to self-monitor behaviour change can be regarded as a form of reinforcement. In line with this, experts stressed the importance of providing a selfmonitoring tool to allow visitors to assess their current health behaviour status and to track their progress. Finally, assessment refers to the website's ability to adjust the website to specific user needs and wishes. Tailoring is considered to be an appropriate strategy to adjust health information to personal characteristics and preferences (Kreuter et al., 2000). Furthermore, tailoring website content to user characteristics is known to positively impact prolonged use of Internet-delivered interventions (Brouwer et al., 2008; Couper et al., 2010; Strecher et al., 2008). In addition, previous studies indicated that providing personalized, iterative feedback regarding one's health behaviour might in itself stimulate revisits to the program (Brouwer et al., 2008). In line with these previous findings, the majority of experts agreed on the importance of stimulating revisits and using iterative feedback.

Development of the Internet-delivered intervention content and its online environment was guided by these results from the Delphi study, complemented by results from the focus groups and the process-evaluation. By considering and incorporating the opinion of potential future users, it was expected that involvement in the intervention would increase, resulting in higher levels of prolonged use (Glasgow et al., 2004). 


\section{Prolonged use of the Internet-delivered intervention}

Prolonged use of the Internet-delivered intervention was closely monitored by studying module initiation and completion. Results indicated that $61 \%$ of all intervention visitors initiated one of the computer tailored modules, with the fruit and vegetable modules being the favourite module. In comparison with people that refrained from initiating one of the CT modules, module initiators were more likely to be male, of older age, employed and had a lower income. Furthermore, with regard to their health behaviour, module initiators were more likely to have a higher body mass index and an unhealthier lifestyle. Results further indicated that $62 \%$ of all module initiators actually completed the module. Predictors of module completion were age, income and health behaviour, with older participants and participants with a higher income and a relatively healthy lifestyle being more likely to complete the module (chapter 5).

Approximately two third of all participants, that obtained a personal overview of their current lifestyle status initiated one of the CT modules, whereas approximately the same percentage of all initiators also completed this module. The number of studies that also assessed module initiation and completion of an Internet-delivered computer tailored lifestyle intervention aimed at the general population, is limited, making it again a challenge to relate the obtained findings to findings from other studies. Estimates of prolonged use of the current intervention were substantially lower compared to a similar Internet-delivered lifestyle intervention that was offered to a comparable sample in a real-life setting. This intervention reported approximate module initiation and completion rates of $99 \%$ and $91 \%$ respectively (Brouwer et al., 2010). A possible explanation for these higher levels of module initiation could be the relatively brief assessment of participants' lifestyle status, as only perceived fat intake, perceived physical activity level and smoking status were assessed to compose a personalized health overview. As our intervention used validated and often extensive questionnaires to measure not only physical activity and smoking, but also fruit and vegetable intake and alcohol consumption, the assessment might have been lengthy and time consuming, causing participants to refrain from initiating one of the CT modules. In addition, the lower levels of module completion could be explained by the extensiveness and thus length of the modules. The comparable intervention used a question to assess intention and subsequently directed participants to either a module regarding their attitude or regarding their self-efficacy. The CT modules used in the current intervention used a fixed gradual approach consisting of four steps to guide people toward behaviour change. By addressing the pros and cons of engaging in the behaviour, the role of significant persons in the direct environment and strategies on how to deal with lack of support and bad role models, preparatory planning and coping planning the modules took considerably more time to complete, which might have 
caused people to refrain from module completion.

Prolonged use of the program was closely monitored and results indicated that people with a relatively unhealthy lifestyle and low-socioeconomic status initiated one of the computer tailored modules. Furthermore, module completers were more likely to have a higher income and a healthier lifestyle. These findings are largely in line with previous studies (Brouwer et al., 2010; Christensen et al., 2009; Spittaels, De Bourdeaudhuij, Brug, et al., 2007; Strecher et al., 2008). The intervention used a computer tailored approach, as this is known to enhance prolonged use of interventions (Brouwer et al., 2008; Couper et al., 2010; Strecher et al., 2008). Information provided in the program was tailored to demographic, cognitive and behavioural characteristics of users (De Vries \& Brug, 1999). As a result, the provided information was expected to be perceived as personally relevant, resulting in intensified elaboration of this information and increased use of the intervention (Kreuter et al., 2000). However, this approach might need further refinement, in order to increase levels of module completion and to engage specific subgroups of intervention users, e.g. people with a low socioeconomic status. A promising strategy might be to use video-based intervention content. Since content of this intervention was predominantly text-based, attractiveness of the intervention might have been limited among people with a low socioeconomic status. Previous research has suggested that using video-based intervention content may be more effective in attracting attention and stimulating comprehension in this specific subgroup (Stanczyk, Crutzen, Bolman, Muris, \& de Vries, 2013). Prolonged use of the intervention might also be further augmented by increasing the level of interactivity within the intervention. Interactivity has been defined as the degree of two-way interaction that is provided in the program (Kiousis, 2002; Noar et al., 2006), for example options for either immediate feedback or reciprocal interaction. Even though the program provided immediate feedback consisting of personalized health information, it did not provide communication features such as support with either a counsellor or with peers. Since recent research has indicated the effects of different types of support on intervention use (Brouwer, Kroeze, et al., 2011; Kelders et al., 2012) specific interactive elements could be added to the Internetdelivered intervention in order to optimize prolonged use.

\section{MAIN FINDINGS RELATED TO SUSTAINED USE}

The third aim of the current project was to optimize sustained use of an Internetdelivered lifestyle intervention by using email prompts to stimulate revisits to the intervention. A periodic email prompt was used to invite people to re-use the intervention in order to self-monitor their level of behaviour change and to benefit from new and updated intervention content and additional computer tailored advice. In 
addition, optimal content and timing of the email prompt was studied in order to further optimize its effect.

\section{The use of a single email prompt after three months}

Positive effects of periodic email prompts were found on sustained use of the Internet-delivered intervention. People that received an additional email, twelve weeks after their first visit, prompting them to revisit the program were more likely to revisit the program compared to people that did not receive an additional prompt (chapter 5). In total, 6\% off all participants that received an email prompt revisited the intervention. Even though this percentage substantially exceeded the proportion of people that revisited the intervention without receiving an additional email prompt, it remained limited, as the proportion of users that responded to this email prompt by revisiting the intervention was still relatively low.

Results from this thesis indicate that prompting visitors to revisit the Internetdelivered computer tailored intervention is an effective strategy to enhance sustained use, although the effect remained limited. A possible explanation for this limited effect was considered to be the period after which the prompt was sent. In the study described in chapter 5 a prompt was sent three months after the first visit to the intervention. This period might have been too long, consequently causing people to forget about the program and their participation in it. This is in line with previous studies that indicated that attrition levels in Internet-delivered interventions are fairly high at the beginning of the intervention period (Bennett \& Glasgow, 2009; Eysenbach, 2005). A second explanation of the limited effect of the email prompts pertained to the content of the prompt. The use of email is becoming more and more popular, not only among Internet users (The Radicati Group, 2011), but also among companies that use email for marketing purposes, without consent from the recipient (Merisavo \& Raulas, 2004; Raad et al., 2010). The receipt of this kind of emails has contributed to a tendency among email users to neglect emails or to delete them before reading (The Radicati Group, 2011). It is therefore important to refine email content to attract people's attention amidst the emails sent for marketing purposes.

\section{Testing optimal timing and content of a single email prompt}

In order to further optimize the effect of the email prompt, we investigated different time intervals and additional prompt content (chapter 6). Firstly, analyses revealed that there were no interaction effects between prompt timing and content. Secondly, analyses of main effects however indicated that the use of shorter prompt intervals increased their impact; sending a prompt two weeks after a first visit resulted in more clicks on the URL presented in the email compared with using a longer time period of 
four or six weeks. This effect was further extended to actually logging in to the intervention, as sending a prompt after two weeks resulted in a significantly higher proportion of program logins compared to six weeks. The effects of adding a preview of new website content to the periodic email prompts did not significantly add to their effects. However, a trend was observed indicating that this added content increased the prompts' effectiveness in persuading people to log in to the program. Thirdly, all experimental groups were compared to a control condition that did not receive any additional emails prompting them to revisit the intervention. Results indicated that only a prompt with additional content that was sent after two weeks, significantly increased program log-ins compared to receiving no additional prompts. The effect of this prompt exceeded the effect of the prompts used in chapter 5, by stimulating $18 \%$ of all visitors to revisit the intervention. In addition, when a preview of new website content was added to this two week standard prompt this proportion increased to 28\% (chapter 6).

Results from this study indicated that using a short prompt interval is beneficial for intervention revisits. This suggests that the interval between engaging in a first visit and receiving an email prompt to boost sustained use of the intervention should be relatively short. Adding a preview of new website content increased the effect of the prompt, but this effect was not significant. Effectiveness of prompt content largely depends on the degree to which the content is actually read and processed by receivers (Petty \& Cacioppo, 1986). Results indicated that people were significantly more likely to respond to emails that were sent at a relatively short interval, irrespective of the email content. One could argue that people simply remember the study and due to their consent to participate in the study were more likely to respond to the email prompt. Studies from the field of email marketing have indicated that when receivers have agreed to the receipt of emails the effects of the emails tend to increase (Barnes \& Scornavacca, 2004; Raad et al., 2010).

In conclusion, we can conclude that sustained use of Internet-delivered interventions can be optimized by using short prompts intervals. Since the costs of sending a large number of email prompts are relatively low (Merisavo \& Raulas, 2004), the use of email prompts can therefore be regarded as a cost-effective strategy to increase sustained use of Internet-delivered interventions. In addition, prompt content should be attuned in order to ensure persuasiveness with regard to sustained use of the intervention.

\section{CONSIDERATIONS}

\section{Representativeness of the current findings}

All studies included in this thesis with regard to intervention reach and use were con- 
ducted using the same Internet-delivered lifestyle intervention. Although this allowed us to compare the different strategies (i.e. the use of different email prompts) and to pronounce upon effects of these strategies, it might limit the generalizability of the obtained results to other Internet-delivered lifestyle interventions. It is therefore important to keep in mind that all obtained results with regard to reach and use should be generalized with caution as interventions with different target behaviours and groups and delivery modes might generate different results.

By using the same Internet-delivered computer tailored intervention in all studies, results obtained with regard to intervention use might also be positively or negatively influenced by evaluation of the intervention by participants. Even though rigorous development of the intervention was emphasized, by developing the intervention in concordance with the opinions of experts and potential future users and conducting focus groups and a process evaluation, this does not warrant satisfaction with the intervention among actual interventions users. Within the present project, all intervention modules were evaluated. Results from chapter 5 indicated that all modules were evaluated positively by module users upon completion. However, in order to evaluate a computer tailored module, people first needed to make use of this module. Hence, evaluation questions were included in the program at the end of each module. As a result, these evaluation questions were only answered by module completers, potentially resulting in bias. Future studies should therefore focus on involving people that refrain from prolonged use in the evaluation of the intervention.

\section{Establishing public health impact of Internet-delivered interventions}

Public health impact of Internet-delivered lifestyle interventions is determined by their efficacy, reach and use among the target population (De Vries \& Brug, 1999; Glasgow et al., 1999). The studies described in this dissertation were part of a greater project. The focus of this thesis was on optimizing reach and use of the intervention (Schneider et al., 2011). The intervention used in this dissertation was an Internetdelivered computer tailored lifestyle intervention, which integrated content from interventions aimed at increasing smoking cessation, promoting the intake of fruit and vegetables, increasing the level of physical activity and reducing the consumption of alcohol (Brug et al., 1999; Poel et al., 2009; Smeets et al., 2008; Van Keulen et al., 2011). Studies aimed at establishing efficacy of the intervention were incorporated in the second part of the project (Schulz et al., 2011) and are therefore not included in this thesis. However, preliminary results from this trial reveal that people who received computer tailored feedback aimed at changing their lifestyle significantly improved their lifestyle compared to people that did not receive additional computer tailored feedback both after twelve and twenty-four months (Schulz et al., submitted). 


\section{Predictors of first, prolonged and sustained use}

By embedding the Internet-delivered intervention in the Adult Health Monitor valuable information was obtained regarding actual numbers of reach of the used dissemination strategy. Furthermore, detailed and extensive information was gathered regarding not only characteristics of people that were interested and participated in the program, but also of people that refrained from participation. This information on user characteristics entailed specific information about demographic characteristics, health behaviour characteristics, mental health status and quality of life, but did not include other characteristics that may influence intervention reach and use. Other characteristics might be cognitive factors, like attitude, self-efficacy and motivation to change (Brouwer et al., 2010; De Vries et al., 2003), user expectations regarding the intervention (Venkatesh, Morris, Davis, \& Davis, 2003) and user experiences (Crutzen et al., 2011). Future studies should therefore focus on precisely determining adequacy of reach by creating more extensive and detailed user profiles. In order to optimize and expand characteristics included in these user profiles, intervention developers should aim at assessing cognitive characteristics, expectations and experiences of intervention users. Assessment of these characteristics can be incorporated in the intervention, for instance by using online questionnaire or diaries. It is therefore imperative to consider assessment of these characteristics during the development phase of the intervention. In addition, future studies should also investigate additional factors that may influence intervention reach and use in order to complement these user profiles. By composing and closely studying profiles of intervention users and by mapping how these people use the intervention, intervention content can be adapted to visitors' needs. This latter is expected to increase use of the intervention, as well as the probability of positive intervention outcomes (Glasgow et al., 2004).

\section{Motives for first, prolonged and sustained use}

The current project provided valuable insights in predictors of interest in the intervention, as well as predictors of actual use. Furthermore, detailed profiles of module initiators and completers were composed. This allowed us to study which people use the program and determine the degree to which they used the program. Furthermore, the online environment of the intervention allowed us to objectively monitor intervention use by tracking users' behaviour at the intervention. This information can subsequently be used to adjust the intervention to further optimize concordance with the needs and wishes of interventions users, by which users' involvement is expected to increase (Cugelman et al., 2011). Consequently, this increased level of involvement might result in increased appreciation of the intervention, lower levels of attrition, and higher levels of prolonged and sustained use. 
It is important, however, to acknowledge that results from this project provide no insights into the reasons users might have to use the intervention the way they decided to use it. Researchers should therefore keep in mind that, even though objectively monitoring program use is important and provides useful insights, human resources are still needed to meaningfully interpret these data (Kaushik, 2007). Program use data should therefore be complemented with process evaluation data obtained through qualitative or quantitative research. These data are valuable, as when objective data tell you which people leave the intervention and when, these data provide relevant information about why people decide to stop using the intervention at this point (Couper et al., 2010). Are people leaving the intervention because it does not meet their wishes and expectations, or are they simply leaving because they have already obtained all relevant information? In order to answer these questions and gain more insight into participants' motives for intervention (non-) use, future research should incorporate qualitative (e.g. focus group interviews) or quantitative (e.g. online questionnaires) process evaluations measures.

\section{RECOMMENTATION FOR PRACTICE AND FUTURE RESEARCH}

\section{Translating the proactive dissemination strategy to other settings and target groups}

The dissemination strategy consisted of embedding the intervention in an existing context; the Adult Health Monitor of the Regional Public Health Services. Even though this strategy can be regarded as successful, future research is needed in order to better interpret the current findings. Firstly, the proactive dissemination strategy was used within the controlled environment of the online Adult Health Monitor. This online Monitor sample could be biased towards participation in an Internet-delivered lifestyle program. It might therefore be desirable to also offer the intervention to participants that decided to fill out the written version of the Adult Health Monitor in order to study whether actual reach and adequacy of reach differs. Secondly, the dissemination strategy was regarded as successful for this specific subgroup of users. In order to further study the impact of proactive dissemination of Internet-delivered lifestyle interventions, the strategy should be translated to other target populations, like specific patient or at-risk groups. Thirdly, within the current project only one dissemination strategy was tested. It is therefore not possible to compare this strategy to other strategies that could be used in the same Monitor environment. These strategies should also focus on persuading people with a relatively unhealthy lifestyle and low socioeconomic status as these people were not optimally reached with the current strategy. Previous research has indicated that these difficult to reach groups might benefit from recruitment through their general practitioner (Brouwer, Oenema, et al., 2011) or through mass-media (Smit et al., 2012). 


\section{Exploring possibilities for proactively disseminating Internet-delivered interventions}

This thesis reports on the findings of using a proactive dissemination strategy to optimize reach of an Internet-delivered computer tailored lifestyle intervention. In order to make an active decision to use an Internet-delivered intervention, people must undergo several steps (De Vries et al., 2003; Weinstein, 1988). Firstly, people need to become aware of the existence of the intervention. After this, they must become aware of their need to use this intervention in order to become motivated to use the intervention. Finally, they must undertake action in order to actually use the intervention and benefit from its content. By using a proactive dissemination approach, the intervention was offered to Adult Health Monitor participants, hereby diminishing important barriers to awareness, like insufficient knowledge on the existence of interventions or places where the intervention can be found (Ajzen, 1991; De Vries et al., 2003; Prochaska et al., 1997). Furthermore, proactively offering Adult Health Monitor participants an opportunity to use a lifestyle intervention can serve as cue to action to become interested in the intervention and actively engage in lifestyle change. It is, therefore, important that intervention developers use proactive strategies to optimize reach of Internet-delivered interventions, as simply making the intervention available on the Internet is unlikely to attract visitors.

In the previous section we have stressed that proactively offering the intervention to potential users is a good strategy, as users themselves do not need to undertake action in order to use the intervention (Prochaska et al., 1997). When proactively offering the intervention, it is important to keep in mind that people are not actively looking for the intervention. As a result interest in the intervention and motivation to actually use the intervention might be relatively low compared to interventions that use a reactive dissemination approach. Besides solely proactively offering the intervention to participants, it is also important to motivate and interest people for the intervention at the same time. Opportunities for proactively disseminating Internetdelivered intervention through the Adult Health Monitor environment should be further optimized in the future. Even though the direct transportation of data obtained from the Adult Health Monitor to the computer tailored lifestyle intervention allowed for composition of an individual health overview addressing all relevant lifestyle behaviours, this overview was only presented to interested participants that decided to actually use the intervention. Impact of the dissemination strategy might therefore be improved by presenting this overview to all Adult Health Monitor participants, instead of only interested participants. Since this overview provides information on the status of individuals' current lifestyle behaviours, as well as on information regarding important guidelines set for these behaviours, people might become aware of their need to use this intervention and subsequently proceed to intervention use. 


\section{Determining when dissemination is successful}

In order to accurately determine reach of Internet-delivered interventions, one must focus on proportions of people exposed to and affected by the dissemination strategy Furthermore, it is important to reflect on adequacy of reach by composing profiles of intervention users and non-users. In order to determine success of the dissemination strategy, the strategy should be compared to other (similar) strategies. However, most Internet trials do not report on these findings, making it difficult to compare dissemination strategies (Dzewaltowski et al., 2004). Future studies into Internetdelivered interventions should therefore aim at monitoring reach of the dissemination strategy and at determining adequacy of reach. In addition, to further enable comparison to other interventions, it is important to advocate the case for incorporating information with regard to reach of interventions in scientific articles and, even more important, in databases where effective interventions are registered (Brug et al., 2010), e.g. the Healthy Living Centre (Centrum Gezond Leven) of the National Institute for Public Health and the Environment (RIVM). Here important information regarding proportions and actual numbers of participants reached, as well as adequacy of reach entailing detailed profiles of users and non-users can be stored and made available to other intervention developers. By combining data of intervention effects with data on intervention reach, intervention developers can make informed decision when selecting both interventions and dissemination strategies, in order to increase public health impact of these interventions (De Vries \& Brug, 1999; Glasgow et al., 1999).

Success of a dissemination strategy can also be determined by comparing indicators of reach and adequacy of reach to a pre-set desired number or proportion of participants that need to be reached. How many people do we want to reach with our interventions? Most intervention developers are inclined to think along the lines of 'the more the merrier'. This line of reasoning may seem logical, since intervention developers either strive to optimize the public health impact of interventions, which is determined by intervention reach and efficacy, or to allow for sufficient power when studying intervention effects in trials (Glasgow et al., 1999). However, in order to pass judgement on the success of the dissemination strategy it is important to define a target reach in advance. Due to the versatility of interventions, dissemination strategies and target populations, it is impossible (and even undesirable) to determine a fixed cut-off point for successful levels of reach. For instance, interventions recruiting smoking participants from a specific patient group via general practice (VonckenBrewster et al., 2013) might require a different relative and or absolute number of participants reached compared to recruiting smoking participants from the general population for an Internet-delivered smoking cessation intervention (Smit et al., 2012). Intervention developers should therefore take into account the accessibility of 
the target group, as well as the prevalence of specific risk behaviours within this target group in order to accurately determine this cut-off point for reach of the intervention in advance.

\section{Monitoring and reporting prolonged use of Internet-delivered interventions}

Within the present study prolonged use of the program was monitored by studying module initiation and completion. It is important to monitor prolonged use of the intervention by assessing users' behaviour within the intervention context (Crutzen, Roosjen, et al., 2012). Obtained information on use provides detailed insight into which elements are used by participants and which elements are neglected or even contribute to early disengagement of in the intervention. This information can subsequently be used to adapt the intervention content to users' implicit intervention needs, again increasing the likelihood of increased appreciation and higher levels of use (Glasgow et al., 2004). Furthermore, this information can be used to compare prolonged use between different Internet-delivered interventions.

Within our studies, objective measures regarding module initiation and completion were used to assess prolonged use of the intervention. The Internet offers substantial possibilities for objectively monitoring behaviour, hereby reducing the necessity to rely on self-reported measures of use, which can be susceptible to users' memory, interpretation and social desirability (Crutzen \& Göritz, 2010, 2011). Even though these measures provided insights into prolonged use of the intervention, this information can be further expanded by including additional measures of prolonged use, like duration of the log in period (Jacobs et al., 2010; Jansen et al., 2008; Leslie et al., 2005; Verheijden et al., 2004), average page view duration for each specific subsection of the intervention session (Lewis et al., 2008), surfing depth on the program website (Jacobs et al., 2010) or the degree to which intervention sessions are initiated and completed (Brouwer et al., 2010; Schulz et al., 2012). There is, however, no 'gold standard' for measuring prolonged use and measures of prolonged use are highly dependent on the structure of the intervention. For interventions with a fixed, tunnelled design measures of prolonged use might include information on the number and duration of page views, whereas for interventions with a freedom-of choice design consisting of several elements (e.g. diaries, questionnaires, self-monitoring etc.) these measures might include information on elements that are used and the degree to which these elements are used. Researchers and intervention developers should therefore report on the specific measures that were used to determine prolonged use, in order to facilitate comparison with results obtained from other interventions. In addition, to take this one step further, it would be good to stimulate full disclosure of research related to intervention use, like intervention manuals, questionnaires and 
data sets (Peters, Abraham, \& Crutzen, 2012). By fully disclosing this information, scrutiny of the research can be maximized. Moreover, it allows for replication of the findings and it facilitates synthesis of the obtained data. This latter is important, as it allows researchers to meta-analyse prolonged use of different interventions, without being dependent on the measures that were published by the researchers.

\section{Using email prompts to optimize sustained intervention use Optimizing prompts interval}

The use of periodic email prompts can be regarded as a successful strategy to increase sustained use of the Internet-delivered computer tailored lifestyle intervention. Results indicated that using a shorter prompt interval increased the effect of the email prompt. Future studies should therefore focus on determining whether even shorter prompt intervals might enhance the effect. When studying prompt intervals it is, for instance, important to keep in mind the purpose of the revisit. When the revisit is aimed at providing additional or new intervention content, shorter prompt intervals might be beneficial. However, one must also allow participants enough time to actually follow up on the obtained advice and develop strategies to positively change their lifestyle. In consequence, when revisits are aimed at self-monitoring, prompt intervals may be longer. Future studies should therefore aim at finding a balance between prompt intervals and the main goal of an intervention revisit.

\section{Moderators of prompt effectiveness}

Even though email prompts were effective in increasing sustained use of the Internetdelivered intervention, these prompts may benefit from further refinement in order to optimize their potential. With regard to characteristics of the prompt, research should focus on optimizing prompt content. Effectiveness of prompt content largely depends on the degree to which the content is actually read and processed by receivers (Petty \& Cacioppo, 1986). Future studies might therefore focus on developing and testing prompt content that is capable of capturing the attention of the receiver, consequently increasing the likelihood that the email will elicit action. Besides changing prompt content, another important step for future research might be to focus on the source (e.g. the sender of the email). Previous studies have indicated that online word of mouth might enhance prompt effects. Information received through word of mouth via friends or family is perceived as more credible, compared to information received from institutions (Crutzen et al., 2009). A third step towards prompts refinement might entail the mode of delivery of prompts, since the medium, e.g. mail, telephone or personal contact, used to deliver prompts may influence their effect (Fry \& Neff, 2009). With regard to characteristics of the receiver, it is important to determine 
which determinants predict responsiveness to email prompts. Even though a comprehensive set of determinants was assessed within the Adult Health Monitor, e.g. demographic characteristics, health behaviour status, and intention to change their health behaviours, the relatively small sample size prevented us from studying predictors of prompt responsiveness. Future studies should therefore aim at distinguishing characteristics of people that tend to react to email prompts. These insights can subsequently be used to tailor prompts to user characteristics, hereby further optimizing their potential.

\section{Using multiple email prompts}

The studies described in this thesis tested the effect of a single email prompt. However, since achieving health behaviour change is an enduring process that requires continuous guidance (Christensen et al., 2009; Norman et al., 2007), repeated revisits might be beneficial (Borland et al., 2004). It is therefore important to study whether repeatedly sending prompts at a certain interval to boost multiple revisits has positive effects, or it this may have reverse effects because responses to multiple prompts may gradually decline over time (Woodall et al., 2007). Additional research should therefore investigate the benefits of expanding the use of single email prompts to multiple prompts and to finding the right balance between multiple email prompts without irritating participants.

\section{Assessing effects on behaviour change}

Besides focussing on prompt efficacy, it is also important to relate the effects of sending periodic email prompts to actual behaviour change. The effects of the prompts used in the current thesis were assessed based on clicking in the URL and on logging in to the intervention. Since there is a strong positive relationship between intervention dose received and intervention effects, one could argue that revisits to the intervention are likely to result in higher levels of intervention effects (Crutzen, de Nooijer, Candel, et al., 2008; Verheijden et al., 2007). However, additional observational research is needed to investigate the actual effects of using periodic prompts on behaviour change and to investigate whether these effects remain present over a sustained period of time.

\section{Cognitive and affective determinants of first, prolonged and sustained intervention use}

The studies described in this thesis were aimed at testing strategies that were used to optimize use of an Internet-delivered computer tailored intervention. These results serve as a good and solid departure point for future research regarding further optimization of reach and use of Internet-delivered interventions. In order to better un- 
derstand prolonged and sustained use of Internet-delivered interventions and to develop effective methods to enhance use, these types of use should be regarded as stand-alone behaviours. By labelling first, prolonged and sustained use as stand-alone behaviours, models can be used to provide useful information regarding determinants of these behaviours. In line with socio-cognitive models that are used to explain behaviour (Ajzen, 1991; De Vries et al., 2003), several factors might influence use of the Internet-delivered intervention, like motivation to use the intervention, expectations towards the (re-) use of the intervention and self-efficacy towards intervention (re-) use. It is important to assess these determinants among intervention users. Future studies should therefore focus on studying and identifying determinants that are related to prolonged and sustained use of interventions. Once important determinants of intervention use are identified, methods and practical applications can be developed that are aimed at positively changing these determinants (Bartholomew, Parcel, Kok, Gottlieb, \& Fernandez, 2011). Arguments and persuasive communication methods can for instance be used to positively change users' attitudes towards use, by emphasizing perceived pros of use and weakening the perceived cons. In addition, planning methods could be included in the intervention, in order to help users to convert their positive intentions towards intervention use into actual action.

By using cognitive theories to develop embedded interventions that are aimed at increasing the motivation to use the program, intervention use is regarded as a deliberate process. However, not all intervention users may be involved in the intervention or motivated to use the intervention. According to the Elaboration Likelihood model (Petty \& Cacioppo, 1986), people with a low level of involvement and motivation are less likely to process arguments used to persuade people to use the intervention. Besides focussing on cognitive determinants, it is therefore also important to focus on affective factors that are related to website use, like user experiences. User experiences entail the thoughts and feelings of a person during and after being exposed to a website (Crutzen et al., 2009b). Literature on user experiences has indicated that besides cognitive perceptions, user experiences also consist of affective perceptions, like enjoyment and trustworthiness (Crutzen et al., 2011). The use of narrative forms of communication, like the use of testimonials or storytelling might be promising strategies to elicit positive user experiences, which may subsequently lead to increased intervention use. Testimonials can be included on websites in order to enable users to share their experiences concerning website use (Crutzen, Cyr, Larios, Ruiter, $\&$ de Vries, 2013). They may increase perceptions of social presence, which refers to the extent to which a website is able to bring about the feeling of human contact and sociability. Previous studies have indicated that the inclusion of social presence elements on websites can positively change attitudes towards the website (Hassanein \& 
Head, 2007), as well as users' intention to use the website (Cyr, Hassanein, Head, \& Ivanov, 2007). Another strategy might entail the use of storytelling. Stories can be used to implicitly inform users about their behaviour and the possible consequences (Larkey \& Gonzalez, 2007). This technique might provide intervention developers valuable opportunities to communicate cognitive information, for instance regarding the importance and possible barriers of intervention use, by incorporating this information in a (fictional) story. Providing intervention users with stories of other (similar) users that are going through the same process of intervention use, might have several important benefits (Green, 2006). Firstly, it can reduce counterarguments, by providing stories of people that overcame important barriers to intervention use. Secondly, it can facilitate the mental simulation of unknown or difficult procedures, like for instance barriers encountered during intervention use. Finally, it can provide important role models for engaging in the desired behaviour, as these stories could display people that were successful in using the intervention. Future studies should therefore also focus on including affect-based strategies to optimize reach and use of Internetdelivered lifestyle interventions. 
References 



\section{A}

Adler, M., \& Ziglio, E. (1996). Gazing into the oracle. Bristol, PA: Jessica Kingsley Publishers.

Ainsworth, B. E., Haskell, W. L., Whitt, M. C., Irwin, M. L., Swartz, A. M., Strath, S. J., O'Brien, W. L., Bassett, D. R., Schmitz, K. H., Emplaincourt, P. O., Jacobs, D. R., \& Leon, A. S. (2000). Compendium of physical activities: an update of activity codes and MET intensities. Medicine \& Science in Sports \& Exercise, 32, s498-504.

Ajzen, A. (1991). The theory of planned behavior. Organizational Behavior and Human Decision Processes, 50, 179-211.

Alcoholinfo.nl. (2012). Aanvaardbaar alcoholgebruik. Retrieved August 5, 2012, from http://www.alcoholinfo.nl/pu bliek/nieuwe-site

Andrews, G., \& Slade, T. (2001). Interpreting scores on the Kessler Psychological Distress Scale (K10). Australian and New Zealand Journal of Public Health, 25, 494-497.

\section{B}

Bansil, P., Keenan, N. L., Zlot, A. I., \& Gilliland, J. C. (2006). Healthrelated information on the Web: results from the HealthStyles Survey, 20022003. Preventing Chronic Disease, 3, A36.

Barnes, S. J., \& Scornavacca, E. (2004). Mobile marketing: the role of permission and acceptance. International Journal of Mobile Communications, 2, 128-139.

Bartholomew, L. K., Parcel, G. S., Kok, G., Gottlieb, N. H., \& Fernandez, M. E. (2011). Planning health promotion programs: an Intervention Mapping approach. (3 ed.). San Fransisco: Jossey-Bass.

Bennett, G. G., \& Glasgow, R. E. (2009). The delivery of public health interventions via the Internet: actualizing their potential. Annual Review of Public Health, 30, 273-292.

Bergstrom, G., Bjorklund, C., Fried, I., Lisspers, J., Nathell, L., Hermansson, U., Helander, A., Bodin, L., \& Jensen, I. B. (2008). A comprehensive workplace intervention and its outcome with regard to lifestyle, health and sick leave: the AHA study. Work, 31, 167-180.

Blumenthal, R., \& Endicott, J. (1998). Barriers to seeking treatment for major depression. Depression and Anxiety, 4, 273-278.

Bogers, R., Van Assema, P., Kester, A., Westerterp, K., \& Dagnelie, P. (2004). Reproducibility, validity, and responsiveness to change of a sort questionnaire for measuring fruit and vegetable intake. American Journal of Epidemiology, 159, 900-909.

Borland, R., Balmford, J., \& Hunt, D. (2004). The effectiveness of personally tailored computer- 
generated advice letters for smoking cessation. Addiction, 99, 369-377.

Brouwer, W., Kroeze, W., Crutzen, R., de Nooijer, J., de Vries, N. K., Brug, J., \& Oenema, A.

(2011). Which intervention characteristics are related to more exposure to Internetdelivered healthy lifestyle promotion interventions? A systematic review. Journal of Medical Internet Research, 13, e2.

Brouwer, W., Oenema, A., Crutzen, R., De Nooijer, J., De Vries, N., \& Brug, J. (2009). What makes people decide to visit and use an Internet-delivered behavior-change intervention?: A qualitative study among adults. Health Education, 109, 460-473.

Brouwer, W., Oenema, A., Crutzen, R., de Nooijer, J., de Vries, N. K., \& Brug, J. (2008). An exploration of factors related to dissemination of and exposure to Internetdelivered behavior change interventions aimed at adults: a Delphi study approach. Journal of Medical Internet Research, 10, e10.

Brouwer, W., Oenema, A., Crutzen, R., De Nooijer, J., de Vries, N. K., \& Brug, J. (2011). Results of distribution of a flyer to attract Dutch adults to an Internet-delivered physical activity promotion intervention: differences between three promotion channels. Improving exposure to Internet-delivered health behaviour change interventions, 153-163.

Brouwer, W., Oenema, A., Raat, H., Crutzen, R., de Nooijer, J., de Vries, N. K., \& Brug, J. (2010). Characteristics of visitors and revisitors to an Internetdelivered computer-tailored lifestyle intervention implemented for use by the general public. Health Education Research, 25, 585595.

Brug, J., Glanz, K., Van Assema, P., Kok, G., \& van Breukelen, G. J. (1998). The impact of computer-tailored feedback and iterative feedback on fat, fruit, and vegetable intake. Health education \& behavior: the official publication of the Society for Public Health Education, 25, 517-531.

Brug, J., Oenema, A., Kroeze, W., \& Raat, H. (2005). The Internet and nutrition education: challenges and opportunities. European journal of clinical nutrition, 59 Suppl 1, S130137; discussion S138-139.

Brug, J., Steenhuis, I., van Assema, P., Glanz, K., \& de Vries, H. (1999). Computer-tailored nutrition education: differences between two interventions. Health Education Research, 14, 249256.

Brug, J., van Dale, D., Lanting, L., Kremers, S., Veenhof, C., Leurs, M., van Yperen, T., \& Kok, G. (2010). Towards evidence-based, quality- 
controlled health promotion: the Dutch recognition system for health promotion interventions. Health Education Research, 25, 1100-1106.

CBS. (2012). Centraal Bureau voor de Statistiek [Statistics Netherlands]: Onderzoek ICT gebruik van huishoudens [Survey ICT use by households]. Retrieved August 5, 2012, from http://statline.cbs.nl/StatWe b/

Centraal Bureau voor de Statistiek. (2011). ICT gebruik van personen naar persoonskenmerken: Centraal Bureau voor de Statistiek. Christensen, H., Griffiths, K. M., \& Farrer, L. (2009). Adherence in Internet interventions for anxiety and depression. Journal of Medical Internet Research, 11, e13.

Cook, C., Heath, F., \& Thompson, R. L. (2000). A meta-analysis of response rates in web- or Internet-based surveys.

Educational and Psychological Measurement, 60, 20-24.

Couper, M. P., Alexander, G. L., Zhang, N., Maddy, N., Nowak, M. A., McClure, J. B., Calvi, J. J., Rolnick, S. J., Stopponi, M. A., \& Cole Johnson, C. (2010). Engagement and retention: measuring breadth and depth of participant use of an online intervention. Journal
Medical Internet Research, 12, e52.

Cremers, H. P., Mercken, L., Oenema, A., \& de Vries, H. (2012). A web-based computertailored smoking prevention programme for primary school children: intervention design and study protocol. BMC Public Health, 12, 277.

Crutzen, R. (2009). Hard to get, hard to keep. Dissemination of and exposure to internetdelivered health behavior change interventions aimed at adolescents. (PhD thesis), Maastricht University, Maastricht.

Crutzen, R. (2010). Optimalisering van de betrokkenheid van deelnemers en datakwaliteit in een cohortstudie [Optimising participants' involvement and data quality in a cohort study]. Maastricht: Maastricht University.

Crutzen, R., Cyr, D., \& de Vries, N. K. (2011). Bringing loyalty to eHealth: theory validation using three Internetdelivered interventions. Journal of Medical Internet Research, 13, e73.

Crutzen, R., Cyr, D., Larios, H., Ruiter, R. A., \& de Vries, N. K. (2013). Social presence and use of Internet-delivered interventions: a multimethod approach. PLoS One, 8, e57067.

Crutzen, R., De Kruif, L., \& De Vries, N. K. (2012). You never get a second change to make a 
first impression: the effect of visual complexity on intention to use websites Interaction Studies, 13, 469477.

Crutzen, R., de Nooijer, J., Brouwer, W., Oenema, A., Brug, J., \& de Vries, N. (2009a).

Effectiveness of online word of mouth on exposure to an Internet-delivered intervention. Psychology \& health, 24, 651-661.

Crutzen, R., de Nooijer, J., Brouwer, W., Oenema, A., Brug, J., \& de Vries, N. K. (2008). Internet-delivered interventions aimed at adolescents: a Delphi study on dissemination and exposure. Health Education Research, 23, 427-439.

Crutzen, R., de Nooijer, J., Brouwer, W., Oenema, A., Brug, J., \& de Vries, N. K. (2009b). A conceptual framework for understanding and improving adolescents' exposure to Internet-delivered interventions. Health promotion international, 24, 277-284.

Crutzen, R., de Nooijer, J., Candel, M. J., \& de Vries, N. K. (2008). Adolescents who intend to change multiple health behaviours choose greater exposure to an internetdelivered intervention. Journal of Health Psychology, 13, 906-911.

Crutzen, R., \& Göritz, A. S. (2010). Social desirability and selfreported health risk behaviors in web-based research: three longitudinal studies. BMC Public Health, 10, 720 .

Crutzen, R., \& Göritz, A. S. (2011). Does social desirability compromise self-reports of physical activity in web-based research? The international journal of behavioral nutrition and physical activity, 8, 31 .

Crutzen, R., Kohl, L., \& de Vries, N. K. (2013). Kennissynthese online preventie Maastricht University. Maastricht, The Netherlands: University Press.

Crutzen, R., Roosjen, J. L., \& Poelman, J. (2012). Using Google Analytics as a process evaluation method for Internet-delivered interventions: an example on sexual health. Health promotion international.

Cugelman, B., Thelwall, M., \& Dawes, P. (2011). Online interventions for social marketing health behavior change campaigns: A metaanalysis of psychological architectures and adherence factors. Journal of Medical Internet Research, 13, e17.

Cunningham, M., Rapee, R., \& Lyneham, H. (2006). Feedback to a prototype selfhelp computer program for anxiety disorders in adolescents. Advances in Mental Health, 5, 216-224.

Cyr, D., Hassanein, K., Head, M., \& Ivanov, A. (2007). The role of social presence in 
establishing loyalty in eservice environments. Interacting with Computers, 19.

\section{D}

Danaher, B. G., Boles, S. M., Akers, L., Gordon, J. S., \& Severson, H. H. (2006). Defining participant exposure measures in Web-based health behavior change programs. Journal of Medical Internet Research, 8, e15.

De Nooijer, J., Oenema, A., Kloek, G., Brug, J., De Vries, H., \& De Vries, N. K. (2005).

Bevordering van gezondheidsgedrag via Internet: nu en in de toekomst [Promotion of healthy behavior through the Internet: now and in the future]. Maastricht: Maastricht University.

De Vet, E., Brug, J., De Nooijer, J., Dijkstra, A., \& De Vries, N. K. (2005). Determinants of forward stage transitions: a Delphi study. Health Education Research, 20, 195205.

De Vries, H., \& Brug, J. (1999). Computer-tailored interventions motivating people to adopt health promoting behaviours: introduction to a new approach. Patient Education and Counseling, 36, 99-105.

De Vries, H., Kremers, S. P. J., Smeets, T., Brug, J., \& Eijmael, K. (2008). The effectiveness of tailored feedback and action plans in an intervention addressing multiple health behaviors. American Journal of Health Promotion, 22, 417425.

De Vries, H., Mudde, A., Leijs, I., Charlton, A., Vartiainen, E., Buijs, G., Clemente, M. P., Storm, H., Gonzalez Navarro, A., Nebot, M., Prins, T., \& Kremers, S. (2003). The European Smoking Prevention Framework Approach (EFSA): an example of integral prevention. Health Education Research, 18, 611626.

De Vries, H., van 't Riet, J., Spigt, M., Metsemakers, J., van den Akker, M., Vermunt, J. K., \& Kremers, S. (2008). Clusters of lifestyle behaviors: results from the Dutch SMILE study. Preventive Medicine, 46, 203208.

Dijkstra, A., \& De Vries, H. (1999). The development of computergenerated tailored interventions. Patient Education and Counseling, 36, 193-203.

Dijkstra, A., De Vries, H., \& Roijackers, J. (1998). Long-term effectiveness of computergenerated tailored feedback in smoking cessation. Health Education Research, 13, 207214.

Dijkstra, A., De Vries, H., Roijackers, J., \& van Breukelen, G. (1998). Tailoring information to enhance quitting in smokers with low motivation to quit: three basic efficacy 
questions. Health Psychology, 17, 513-519.

Dijkstra, A., Roijackers, J., \& De Vries, H. (1998). Smokers in four stages of readiness to change. Addictive Behaviors, 23, 339-350.

DSS Research. (2011). Researcher's toolkit. Retrieved May 26, 2011, from https://www.dssresearch.co $\mathrm{m} /$ KnowledgeCenter/toolkitc alculators

Dzewaltowski, D. A., Glasgow, R. E., Klesges, L. M., Estabrooks, P. A., \& Brock, E. (2004). REAIM: evidence-based standards and a Web resource to improve translation of research into practice. Annals of Behavioral Medicine, 28, 75-80.

\section{5}

Elfeddali, I., Bolman, C., \& de Vries, H. (2012). SQ4U - A computer tailored smoking relapse prevention program incorporating planning strategy assignments and multiple feedback time points after the quit-attempt: development and design protocol. Contemporary Clinical Trials, 33, 151-158.

Elfeddali, I., Bolman, C., Mesters, I., Wiers, R. W., \& de Vries, H. (2010). Factors underlying smoking relapse prevention: results of an international Delphi study. Health Education Research, 25, 1008-1020.
Etter, J. F. (2005). Comparing the efficacy of two Internetbased, computer-tailored smoking cessation programs: a randomized trial. Journal of Medical Internet Research, 7, e2.

Evers, K. E., Cummins, C. O., Prochaska, J. O., \& Prochaska, J. M. (2005). Online health behavior and disease management programs: are we ready for them? Are they ready for us? Journal of Medical Internet Research, 7, e27.

Evers, K. E., Prochaska, J. M., Prochaska, J. O., Driskell, M. M., Cummins, C. O., \& Velicer, W. F. (2003). Strengths and weaknesses of health behavior change programs on the Internet. Journal of Health Psychology, 8, 63-70.

Eysenbach, G. (2005). The law of attrition. Journal of Medical Internet Research, 7, e11.

Eysenbach, G., \& Kohler, C. (2002). How do consumers search for and appraise health information on the world wide web? Qualitative study using focus groups, usability tests, and in-depth interviews. BMJ, 324, 573577.

Eysenbach, G., \& Kohler, C. (2003). What is the prevalence of health-related searches on the World Wide Web? Qualitative and quantitative analysis of search engine queries on the Internet. Paper 
presented at the AMIA

Annual Symposium.

Eysenbach, G., Powell, J., Kuss, O., \&

Sa, E. R. (2002). Empirical

studies assessing the quality

of health information for

consumers on the world wide

web: a systematic review.

JAMA, 287, 2691-2700.

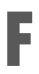

FlyCatcher. (2011). FlyCatcher Internet

Research. Retrieved June 6, 2011, from

http://www.flycatcher.eu/

Fry, J. P., \& Neff, R. A. (2009). Periodic prompts and reminders in health promotion and health behavior interventions: systematic review. Journal of Medical Internet Research, 11, e16.

Galesic, M., \& Bosjnak, M. (2009).

Effects of Questionnaire Length on Participation and Indicators of Response Quality in a Web Survey. Public Opinion Quarterly, 73, 349-360.

Gezondheidsraad. (2006). Richtlijnen geode voeding achtergronddocument [Guidelines for good nutrition - background material]. Den Haag.

GGD Nederland. (2011). GGD

Nederland: Lokale en

Nationale Monitor Gezondheid. Retrieved May 26, 2011, from http://www.monitorgezondh eid.nl
GGD Nederland. (2012). Regionaal Kompas Volksgezondheid. Retrieved August 5, 2012, from http://www.regionaalkompas .nl/

Glasgow, R. (2007). eHealth Evaluation and Dissemination Research.

American Journal of

Preventive Medicine, 32, 119126.

Glasgow, R., Kleges, L., Dzewaltowski, D., Bull, S., \& Estabrooks, P. (2004). The future of health behavior change research: What is needed to improve translation of research into health promotion practice? Annals of Behavioral Medicine, 27, 1-11.

Glasgow, R. E., Christiansen, S. M., Kurz, D., King, D. K., Woolley, T., Faber, A. J., Estabrooks, P. A., Strycker, L., Toobert, D., \& Dickman, J. (2011).

Engagement in a diabetes self-management website: usage patterns and generalizability of program use. Journal of Medical Internet Research, 13, e9.

Glasgow, R. E., Nelson, C. C., Kearney, K. A., Reid, R., Ritzwoller, D. P., Strecher, V. J., Couper, M. P., Green, B., \& Wildenhaus, K. (2007). Reach, engagement, and retention in an Internet-based weight loss program in a multi-site randomized controlled trial. Journal of Medical Internet Research, 9, e11.

Glasgow, R. E., Vogt, T. M., \& Boles, S. M. (1999). Evaluating the 
public health impact of

health promotion

interventions: the RE-AIM

framework. American Journal

of Public Health, 89, 1322-

1327.

Göritz, A. S., \& Crutzen, R. (2012).

Reminders in web-based data

collection: Increasing

response at the price of

retention. American Journal

of Evaluation, 33, 240-250.

Graham, A. L., Bock, B. C., Cobb, N. K., Niaura, R., \& Abrams, D. B. (2006). Characteristics of smokers reached and recruited to an Internet smoking cessation trial: a case of denominators.

Nicotine \& Tobacco Research, 8 suppl 1, S43-48.

Green, M. C. (2006). Narratives and

Cancer Communication. Journal of Communication, 56, 163-183.

Griffiths, F., Lindenmeyer, A., Powell, J., Lowe, P., \& Thorogood, M. (2006). Why are health care interventions delivered over the Internet? A systematic review of the published literature. Journal of Medical Internet Research, 8, e10.

\section{H}

Harvey Berino, J., Pintauro, S., Buzzell, P., DiGiulio, M., Casey Gold, B., Moldovan, C., \& Ramirez, E. (2002). Does using the Internet facilitate the maintenance of weight loss? International Journal of Obesity \& Related Metabolic Disorders, 26, 1254-1260.
Hassanein, K., \& Head, M. (2007). Manipulating perceived social presence through the web interface and its impact on attitude towards online shopping. International Journal of Human-Computer Studies, 65, 689-708.

Heatherton, T. F., Kozlowski, L. T., Frecker, R. C., \& Fagerstrom, K. O. (1991). The Fagerstrom Test for Nicotine Dependence: a revision of the Fagerstrom Tolerance Questionnaire. British Journal of Addiction, 86, 1119-1127.

Heesch, K. C., Masse, L. C., Dunn, A. L., Frankowski, R. F., \& Mullen, P. D. (2003). Does adherence to a lifestyle physical activity intervention predict changes in physical activity? Journal of Behavioral Medicine, 26, 333348.

Hildebrandt, V. H., Chorus, A. M. J., \& Stubbe, J. H. (2010). Trendrapport Bewegen en Gezondheid 2008-2009. In T. K. v. Leven (Ed.). Leiden.

Hong, T. (2006). Contributing factors to the use of health-related websites. Journal of Health Communication, 11, 149-165.

Houston, T. K., Coley, H. L., Sadasivam, R. S., Ray, M. N., Williams, J. H., Allison, J. J., Gilbert, G. H., Kiefe, C. I., Kohler, C., \& Group, D. C. (2010). Impact of content-specific email reminders on provider participation in an online intervention: a dental PBRN study. Studies in Health 
Technology and Informatics, 160, 801-805.

Internet World Stats (2013). Internet usage statistics: The Internet big picture. Retrieved January 14, 2013 from http://www.internetworldsta ts.com/stats.htm

Jacobs, N., De Bourdeaudhuij, I., \& Claes, N. (2010). Surfing depth on a behaviour change website: predictors and effects on behaviour. Informatics for Health and Social Care, 35, 41-52. Jansen, W., Raat, H., Zwanenburg, E., Reuvers, I., van Walsem, R., \& Brug, J. (2008). A schoolbased intervention to reduce overweight and inactivity in children aged $6-12$ years: study design of a randomized controlled trial. BMC Public Health, 8, 257.

Jenkinson, C., Layte, R., Jenkinson, D., Lawrence, K., Petersen, S., Paice, C., \& Stradling, J. (1997). A shorter form health survey: can the SF-12 replicate results from the SF36 in longitudinal studies? Journal of Public Health Medicine, 19, 179-186.

Kaushik, A. (2007). Web Analytics: An Hour a Day. Indianapolis, IN: Wiley Publishing, Inc.

Kelders, S. M., Kok, R. N., Ossebaard, H. C., \& Van Gemert-Pijnen, J.
E. (2012). Persuasive system design does matter: a systematic review of adherence to web-based interventions. Journal of Medical Internet Research, 14, e152.

Kessler, R. C., Andrews, G., Colpe, L. J., Hiripi, E., Mroczek, D. K., Normand, S. L., Walters, E. E., \& Zaslavsky, A. M. (2002). Short screening scales to monitor population prevalences and trends in non-specific psychological distress. Psychological Medicine, 32, 959-976.

Kiousis, S. (2002). Interactivity: a concept explication. New Media \& Society, 4, 355-383.

Kohl, L. F. M., Crutzen, R., \& de Vries, N. K. (2013). Online prevention aimed at lifestyle behaviors: A systematic review of reviews. Journal of Medical Internet Research, 15, e146.

Kongsved, S. M., Basnov, M., HolmChristensen, K., \& Hjollund, N. H. (2007). Response rate and completeness of questionnaires: a randomized study of Internet versus paper-and-pencil versions. Journal of Medical Internet Research, 9, e25.

Krebs, P., Prochaska, J. O., \& Rossi, J. S. (2010). A meta-analysis of computer-tailored interventions for health behavior change. Preventive Medicine, 51, 214-221.

Kreuter, M., Farrell, D., Olevitch, L., \& Brennan, L. (2000). Tailoring 
Health Messages:

Customizing Communication with Computer Technology.

Mahwah, NJ: Erlbaum.

Kwaliteitsinstituut voor de

Gezondheidszorg CBO.

(2004). Richtlijn Behandeling

van tabaksverslaving [Directe

treatment of tobacco

addiction]. In

Kwaliteitsinstituut voor de

Gezondheidszorg CBO (Ed.).

Larkey, L. K., \& Gonzalez, J. (2007).

Storytelling for promoting colorectal cancer prevention and early detection among Latinos. Patient Educ Couns, 67, 272-278.

Lemmens, P., Tan, E. S., \& Knibbe, R. A. (1992). Measuring quantity and frequency of drinking in a general population survey: a comparison of five indices. Journal of studies on alcohol and drugs, 53, 476-486.

Leslie, E., Marshall, A. L., Owen, N., \& Bauman, A. (2005).

Engagement and retention of participants in a physical activity website. Preventive Medicine, 40, 54-59.

Lewis, B., Williams, D., Dunsiger, S., Sciamanna, C., Whiteley, J., Napolitano, M., Bock, B., Jakicic, J., Getz, M., \& Marcus, B. (2008). User attitudes towards physical activity websites in a randomized controlled trial. Preventive Medicine, 47, 508513.
Lindgaard, G., Fernandes, G., Dudek, C., \& Brown, J. (2006).

Attention web designers: You have 50 milliseconds to make a good first impression! Behaviour \& Information Technology, 25, 115-126.

Linstone, H. A., \& Turoff, M. (1975). Introduction to the Delphi method: techniques and applications The Delphi Method: Techniques and Applications (pp. 3-12). Reading, MA: AddisonWesley Publishing Company.

Lorence, D., \& Park, H. (2008). Group disparities and health information: a study of online access for the underserved. Health Informatics Journal, 14, 29-38.

Lustria, M. L. A. (2007). Can interactivity make a difference? Effects of interactivity on the comprehension of and attitudes toward online health content: Research Articles. Journal of the American Society for Information Science and Technology, 58, 766-776.

Lustria, M. L. A., Cortese, J., Noar, S. M., \& Glueckauf, R. L. (2009). Computer-tailored health interventions delivered over the Web: review and analysis of key components.

Patientient Education and Counseling, 74, 156-173.

\section{M}

Menon, S., \& Soman, D. (2002). Managing the Power of 
Curiosity for Effective Web

Advertising Strategies.

Journal of Advertising, 31, 1-

14.

Merisavo, M., \& Raulas, M. (2004).

The impact of email

marketing on brand loyalty.

Journal of Product \& Brand

Management, 13, 498-505.

Moorman, C., \& Matulich, E. (1993). A

Model of Consumers'

Preventive Health Behaviors:

The Role of Health

Motivation and Health

Ability. Journal of Consumer

Research Inc., 20, 208-228.

Mullen, P. M. (2003). Delphi: myths and reality. Emerald / Journal of Health Organization and Management, 17, 37-52.

Myung, S. K., McDonnell, D. D., Kazinets, G., Seo, H. G., \& Moskowitz, J. M. (2009).

Effects of Web- and computer-based smoking cessation programs: metaanalysis of randomized controlled trials. Archives of Internal Medicine, 169, 929937.

Neville, L. M., O'Hara, B., \& Milat, A. J. (2009). Computer-tailored dietary behaviour change interventions: a systematic review. Health Education Research, 24, 699-720.

Noar, S. M., Clark, A., Cole, C., \& Lustria, M. L. (2006). Review of interactive safer sex Web sites: practice and potential. Health Communication, 20, 233-241.
Norman, G. J., Zabinski, M. F., Adams, M. A., Rosenberg, D. E., Yaroch, A. L., \& Atienza, A. A. (2007). A review of eHealth interventions for physical activity and dietary behavior change. American Journal of Preventive Medicine, 33, 336345.

Peters, G. Y., Abraham, C., \& Crutzen, C. (2012). Full disclosure: doing behavioural science necessitates sharing. The European Health Psychologist, 14, 77-84.

Petty, R. E., \& Cacioppo, J. T. (1986). The elaboration likelihood of persuasion. Advances in Experimental Social Psychology, 19, 123-205.

Poel, F., Bolman, C., Reubsaet, A., \& de Vries, H. (2009). Efficacy of a single computer tailored mail for smoking cessation: results after six months. Health Education Research, 24, 930940.

Portnoy, D. B., Scott-Sheldon, L. A., Johnson, B. T., \& Carey, M. P. (2008). Computer-delivered interventions for health promotion and behavioral risk reduction: a metaanalysis of 75 randomized controlled trials, 1988-2007. Preventive Medicine, 47, 316.

Prochaska, J. O., \& DiClemente, C. C. (1983). Stages and processes of self-change of smoking: toward an integrative model of change. Journal of 
Consulting and Clinical Psychology, 51, 390-395. Prochaska, J. O., Redding, C. A., \& Evers, K. E. (1997a). The transtheoretical model and stages of change. In K. Glanz (Ed.), Health Behavior and Health Education: Theory, Research, and Practice. (pp. 99-120). San Fransisco: Jossey-Bass.

Prochaska, J. O., Velicer, W. F., Fava, J. L., Rossi, J. S., \& Tsoh, J. Y. (2001). Evaluating a population-based recruitment approach and a stage-based expert system intervention for smoking cessation. Addictive Behaviors, 26, 583-602.

\section{$\mathbf{R}$}

Raad, M., Yeassen, N. M., Alam, G. M., Zaidan, B. B., \& Zidan, A. A. (2010). Impact of spam advertisement through email: A study to assess the influence of the anti-spam on the e-mail marketing. African Journal of Business Management, 4, 2362-2367.

Riper, H., Kramer, J., Smit, F., Conijn, B., Schippers, G., \& Cuijpers, P. (2008). Web-based selfhelp for problem drinkers: a pragmatic randomized trial. Addiction, 103, 218-227.

Ritterband, L. M., Gonder-Frederick, L. A., Cox, D. J., Clifton, A. D., West, R. W., \& Borowitz, S. (2003). Internet interventions: In review, in use and into the future. Professional Psychology:
Research and Practice, 34, 527-534.

Ritterband, L. M., \& Thorndike, F. (2006). Internet interventions or patient education web sites? Journal of Medical Internet Research, 8, e18; author reply e19.

Ritterband, L. M., Thorndike, F. P., Cox, D. J., Kovatchev, B. P., \& Gonder-Frederick, L. A. (2009). A behavior change model for Internet interventions. Annals of Behavioral Medicine, 38, 1827.

RIVM. (2005). Lokale en nationale Monitor gezondheid. Indicatoren voor de Monitor Volksgezondheid [Local and national Health Monitor. Indicators of the Public Health Monitor].

RIVM. (2008). Volksgezondheid Toekomst Verkenning: Nationaal Kompas Volksgezondheid. Bilthoven, The Netherlands: RIVM.

RIVM. (2012). Volksgezondheid Toekomst Verkenning: Nationaal Kompas Volksgezondheid. Bilthoven, The Netherlands: RIVM.

Rogers, E. (1995). Diffusion of Innovations. New York, NY: The free press.

Roos, G., Johansson, L., Kasmel, A., Klumbiene, J., \& Prattala, R. (2001). Disparities in vegetable and fruit consumption: European cases from the north to the south. Public health nutrition, 4, 35-43. 


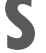

Schneider, F., van Osch, L., \& de Vries, H. (2012). Identifying factors for optimal development of health-related websites: a delphi study among experts and potential future users. Journal of Medical Internet Research, 14, e18.

Schneider, F., van Osch, L., Schulz, D. N., Kremers, S. P., \& de Vries, H. (2012). The influence of user characteristics and a periodic email prompt on exposure to an Internetdelivered computer-tailored lifestyle program. J Med Internet Res, 14, e40.

Schneider, F., van Osch, L. A., Kremers, S. P., Schulz, D. N., van Adrichem, M. J., \& de Vries, H. (2011). Optimizing diffusion of an online computer tailored lifestyle program: a study protocol. BMC Public Health, 11, 480.

Schulz, D. N., Kremers, S. P., van Osch, L. A., Schneider, F., van Adrichem, M. J., \& de Vries, H. (2011). Testing a Dutch web-based tailored lifestyle programme among adults: a study protocol. BMC Public Health, 11, 108.

Schulz, D. N., Kremers, S. P., Vandelanotte, C., Van Adrichem, M. J. G., Schneider, F., Candel, M. J. J. M., \& de Vries, $H$. (submitted). Effects of a webbased tailored multiple lifestyle intervention for adults: A two-year randomized controlled trial comparing sequential and simultaneous delivery modes.

Schulz, D. N., Schneider, F., de Vries, H., van Osch, L. A., van Nierop, P. W., \& Kremers, S. P. (2012). Program completion of a web-based tailored lifestyle intervention for adults: differences between a sequential and a simultaneous approach. Journal of Medical Internet Research, 14, e26.

Shahab, L., \& McEwen, A. (2009). Online support for smoking cessation: a systematic review of the literature. Addiction, 104, 1792-1804.

Smeets, T., Brug, J., \& de Vries, H. (2008). Effects of tailoring health messages on physical activity. Health Education Research, 23, 402-413.

Smeets, T., Kremers, S. P., Brug, J., \& de Vries, H. (2007). Effects of tailored feedback on multiple health behaviors. Annals of Behavioral Medicine, 33, 117123.

Smit, E. S., de Vries, H., \& Hoving, C. (2010). The PAS study: a randomized controlled trial evaluating the effectiveness of a web-based multiple tailored smoking cessation programme and tailored counselling by practice nurses. Contemporary Clinical Trials, 31, 251-258.

Smit, E. S., Hoving, C., Cox, V. C., \& de Vries, H. (2012). Influence of recruitment strategy on the reach and effect of a web- 
based multiple tailored

smoking cessation

intervention among Dutch

adult smokers. Health

Education Research, 27, 191-

199.

Spittaels, H., \& De Bourdeaudhuij, I.

(2007). Who participates in a

computer-tailored physical

activity program delivered

through the Internet? A

comparison of participants'

and non-participants'

characteristics. International

Journal of Behavioral

Nutrition Physical Activity, 4, 39.

Spittaels, H., De Bourdeaudhuij, I., Brug, J., \& Vandelanotte, C. (2007). Effectiveness of an online computer-tailored physical activity intervention in a real-life setting. Health Education Research, 22, 385396.

Spittaels, H., De Bourdeaudhuij, I., \& Vandelanotte, C. (2007). Evaluation of a websitedelivered computer-tailored intervention for increasing physical activity in the general population.

Preventive Medicine, 44, 209217.

Stanczyk, N. E., Crutzen, R., Bolman, C., Muris, J. W., \& de Vries, H. (2013). Influence of delivery strategy on messageprocessing mechanisms and future adherence to a Dutch computer-tailored smoking cessation intervention. Journal Medical Internet Research, 15, e28.
Steyaert, J., \& de Haan, J. (2007). Jaarboek ICT en samenleving 2007: Gewoon digitaal [Yearbook ICT and society 2007: Simply digital]. Amsterdam, The Netherlands: Boom.

Strecher, V., Wang, C., Derry, H., Wildenhaus, K., \& Johnson, C. (2002). Tailored interventions for multiple risk behaviors. Health Education Research, 17, 619-626.

Strecher, V. J. (1999). Computertailored smoking cessation materials: a review and discussion. Patient Education and Counseling, 36, 107-117.

Strecher, V. J., McClure, J., Alexander, G., Chakraborty, B., Nair, V., Konkel, J., Greene, S., Couper, M., Carlier, C., Wiese, C., Little, R., Pomerleau, C., \& Pomerleau, O. (2008). The role of engagement in a tailored web-based smoking cessation program: randomized controlled trial. Journal of Medical Internet Research, 10, e36.

Svensson, M., Svensson, T., Hansen, A. W., \& Trolle Lagerros, Y. (2012). The effect of reminders in a web-based intervention study. European Journal of Epidemiology, 27, 333-340.

The Radicati Group. (2011). E-mail statistics report: 2011-2015. Palo Alto, CA. 
Tognazzini, B. (2011). Ask TOG: First principles of interaction design. Retrieved June 6, 2011, from http://www.asktog.com/basi cs/firstPrinciples.html

Tu, H. T., \& Cohen, G. R. (2008). Striking jump in consumers seeking health care information. Tracking Report, 20, 1-8.

\section{V}

Van 't Riet, J., Crutzen, R., \& De Vries, H. (2010). Investigating predictors of visiting, using, and revisiting an online health-communication program: a longitudinal study. Journal of Medical Internet Research, 12, e37.

Van Assema, P., Brug, J., Ronda, G., Steenhuis, I., \& Oenema, A. (2002). A short dutch questionnaire to measure fruit and vegetable intake: relative validity among adults and adolescents. Nutrition and Health, 16, 85-106.

Van den Berg, M. H., Schoones, J. W., \& Vliet Vlieland, T. P. (2007). Internet-based physical activity interventions: a systematic review of the literature. Journal of Medical Internet Research, 9, e26.

Van Keulen, H. M., Mesters, I., Ausems, M., van Breukelen, G., Campbell, M., Resnicow, K., Brug, J., \& de Vries, H. (2011). Tailored print communication and telephone motivational interviewing are equally successful in improving multiple lifestyle behaviors in a randomized controlled trial. Annals of Behavioral Medicine, 41, 104-118.

Van Keulen, H. M., Mesters, I., Brug, J., Ausems, M., Campbell, M., Resnicow, K., Zwietering, P. J., van Breukelen, G., van Mechelen, W., Severens, J. L., \& de Vries, H. (2008). Vitalum study design: RCT evaluating the efficacy of tailored print communication and telephone motivational interviewing on multiple health behaviors. BMC Public Health, 8, 216.

Vandelanotte, C., De Bourdeaudhuij, I., Sallis, J. F., Spittaels, H., \& Brug, J. (2005). Efficacy of sequential or simultaneous interactive computer-tailored interventions for increasing physical activity and decreasing fat intake. Annals of Behavioral Medicine, 29, 138-146.

Vandelanotte, C., Spathonis, K. M., Eakin, E. G., \& Owen, N. (2007). Website-delivered physical activity interventions a review of the literature. American Journal of Preventive Medicine, 33, 5464.

Velicer, W. F., Prochaska, J. O., Fava, J. L., Laforge, R. G., \& Rossi, J. S. (1999). Interactive versus noninteractive interventions and dose-response relationships for stagematched smoking cessation programs in a managed care 
setting. Health Psychology, 18, 21-28.

Venkatesh, V., Morris, M. G., Davis, G. B., \& Davis, F. D. (2003). User acceptance of information technology: Toward a unified view. MIS Quarterly, 27, 425478.

Verheijden, M., Bakx, J. C., Akkermans, R., van den Hoogen, H., Godwin, N. M., Rosser, W., van Staveren, W., \& van Weel, C. (2004). Web-based targeted nutrition counselling and social support for patients at increased cardiovascular risk in general practice: randomized controlled trial. Journal of Medical Internet Research, 6, e44.

Verheijden, M. W., Jans, M. P., Hildebrandt, V. H., \& Hopman-Rock, M. (2007). Rates and determinants of repeated participation in a web-based behavior change program for healthy body weight and healthy lifestyle. Journal of Medical Internet Research, 9, e1.

Villar, A., Callegro, M., \& Yang, Y. (2013). Where am I? A metaanalysis of experiments on the effects of progress indicators for web surveys. Social Science Computer Review, in press.

Voncken-Brewster, V., Tange, H., de Vries, H., Nagykaldi, Z., Winkens, B., \& van der Weijden, T. (2013). A randomised controlled trial testing a web-based, computer-tailored selfmanagement intervention for people with or at risk for chronic obstructive pulmonary disease: a study protocol. BMC Public Health, 13, 557.

\section{W}

Wangberg, S. C., Bergmo, T. S., \& Johnsen, J. K. (2008).

Adherence in Internet-based interventions. Patient Preference and Adherence, 2, 57-65.

Wanner, M., Martin-Diener, E., Bauer, G., Braun-Fahrlander, C., \& Martin, B. W. (2010). Comparison of trial participants and open access users of a web-based physical activity intervention regarding adherence, attrition, and repeated participation. Journal of Medical Internet Research, 12, e3.

Ware, J. E., Kosinski, M., \& Keller, S. D. (1996). A 12-item Short-Form Health Survey: construction of scales and preliminary tests of reliability and validity. Medical Care, 34, 220-233.

Weinstein, N. D. (1988). The precaution adoption process. Health Psychology, 7, 355386.

Weinstein, P. K. (2006). A review of weight loss programs delivered via the Internet. Journal of Cardiovascular Nursing, 21, 251-258; quiz 259-260. 
Wendel Vos, G. C., Schuit, A. J., Saris, W. H., \& Kromhout, D. (2003). Reproducibility and relative validity of the short questionnaire to assess health-enhancing physical activity. Journal of clinical epidemiology, 56, 1163-1169.

White, A., Kavanagh, D., Stallma, H., Klein, B., Kay-Lambkin, F., Proudfoot, J., Drennan, J., Connor, J., Baker, A., Hines, E., \& Young, R. (2010). Online Alcohol Interventions: A Systematic Review. Journal of Medical Internet Research, 12, e62.

Whittlesea, B., Jacoby, J. L., \& Girardi, K. (1990). Illusions of immediate memory: Evidence of an attributional basis for feelings of familiarity and perceptual quality. Journal of Memory and Language, 29, 716-732. Woodall, W. G., Buller, D. B., Saba, L., Zimmerman, D., Waters, E., Hines, J. M., Cutter, G. R., \& Starling, R. (2007). Effect of emailed messages on return use of a nutrition education website and subsequent changes in dietary behavior. Journal of Medical Internet Research, 9, e27.

World Health Organisation. (2005). The Bangkok Charter for Health Promotion in a Globalized World (WHO Ed.). Geneva, Switserland: WHO.

World Health Organisation. (2011). The top ten causes of death. WHO fact sheet. Geneva.

World Health Organisation. (2013). Noncommunicable diseases. WHO fact sheet. Geneva.

Yang, S., Lynch, J., Schulenberg, J., Roux, A. V., \& Raghunathan, T. (2008). Emergence of socioeconomic inequalities in smoking and overweight and obesity in early adulthood: the national longitudinal study of adolescent health. American Journal of Public Health, 98, 468-477. 


\section{Appendices}





\section{APPENDIX 1 - RESULTS DELPHI STUDY}

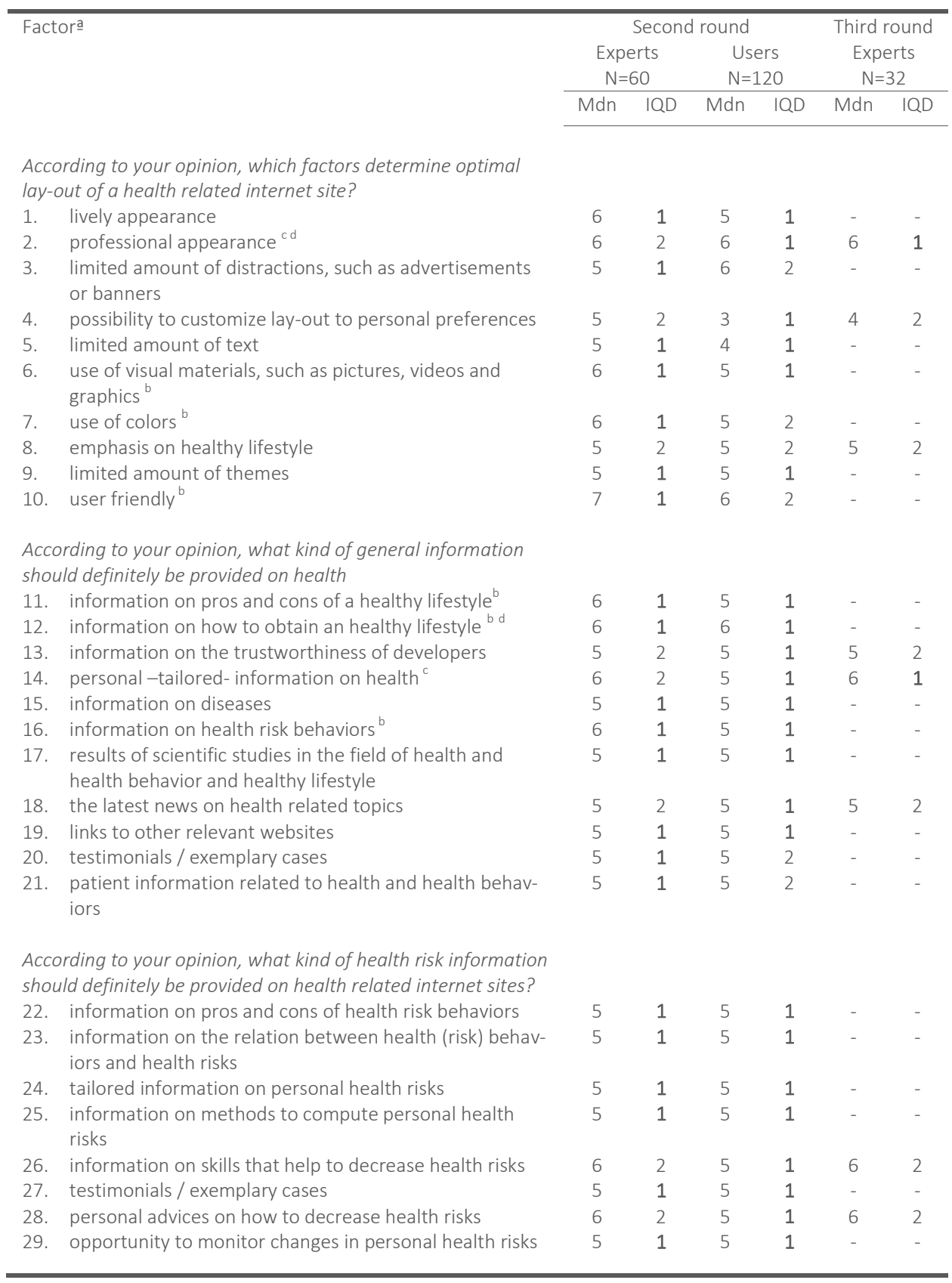




\begin{tabular}{|c|c|c|c|c|c|c|c|}
\hline \multirow{3}{*}{\multicolumn{2}{|c|}{ Factor $\underline{a}$}} & \multicolumn{4}{|c|}{ Second round } & \multirow{2}{*}{\multicolumn{2}{|c|}{$\begin{array}{c}\text { Third round } \\
\text { Experts } \\
N=32\end{array}$}} \\
\hline & & \multicolumn{2}{|c|}{$\begin{array}{l}\text { Experts } \\
\mathrm{N}=60\end{array}$} & \multicolumn{2}{|c|}{$\begin{array}{c}\text { Users } \\
\mathrm{N}=120\end{array}$} & & \\
\hline & & Mdn & IQD & Mdn & IQD & Mdn & IQD \\
\hline 30. & $\begin{array}{l}\text { positive reinforcement in case of low or decreased } \\
\text { health risks }\end{array}$ & 5 & 1 & 5 & 1 & - & - \\
\hline 31. & information on prevalence rates of common diseases & 5 & 2 & 5 & 1 & 5 & 2 \\
\hline 32. & information compromising visual aids, e.g. graphs ${ }^{b}$ & 6 & 1 & 5 & 1 & - & - \\
\hline 33. & $\begin{array}{l}\text { information on professional organizations in the field of } \\
\text { health risks }\end{array}$ & 5 & 2 & 5 & 1 & 5 & 2 \\
\hline 34. & $\begin{array}{l}\text { information on specific health (risk) behaviors related to } \\
\text { health risks }\end{array}$ & 5 & 1 & 5 & 1 & - & - \\
\hline 35. & information on the trustworthiness of developers & 5 & 0 & 5 & 1 & - & - \\
\hline & $\begin{array}{l}\text { information on health risk status of relevant others to } \\
\text { allow for comparison }\end{array}$ & 5 & 1 & 5 & 2 & - & - \\
\hline 37. & personal feedback on potential changes in health risks & 5 & 1 & 5 & 2 & - & - \\
\hline
\end{tabular}

Which factors determine optimal ease of use of health related internet sites, according to your opinion?

\begin{tabular}{|c|c|c|c|c|c|}
\hline 38. clear structure $^{b} \ddagger$ & 7 & 1 & 6 & 1 & - \\
\hline 39. availability of a forum & 4 & 2 & 5 & 1 & 4 \\
\hline availability of a chat function & 4 & 1 & 4 & 2 & 5 \\
\hline $\begin{array}{l}\text { availability of interactive components, e.g. games and } \\
\text { test }\end{array}$ & 5 & 2 & 4 & 2 & 5 \\
\hline limited amount of links to other (relevant) websites & 4 & 1 & 4 & 1 & - \\
\hline $\begin{array}{l}\text { availability of a library containing relevant information } \\
\text { on health and health behaviors }\end{array}$ & 5 & 2 & 5 & 1 & 5 \\
\hline $\begin{array}{l}\text { availability of a function to customize the site for per- } \\
\text { sonal needs }{ }^{b}\end{array}$ & 6 & 1 & 4 & 2 & - \\
\hline availability of contact information from developers ${ }^{c}$ & 6 & 2 & 5 & 1 & 6 \\
\hline availability of an easy log-in procedure ${ }^{b d}$ & 7 & 1 & 6 & 1 & - \\
\hline availability of a helpdesk ${ }^{b}$ & 6 & 1 & 5 & 2 & - \\
\hline little amount of scrolling required to navigate the site & 5 & 1 & 5 & 2 & - \\
\hline use of comprehensive language ${ }^{b}$ & 7 & 1 & 6 & 2 & - \\
\hline availability of a search engine & 6 & 2 & 6 & 2 & 6 \\
\hline availability of bread crumb navigation $^{d}$ & 5 & 2 & 6 & 1 & 5 \\
\hline $\begin{array}{l}\text { limited amount if distractions, such as advertisements } \\
\text { and pop-ups }\end{array}$ & 6 & 2 & 6 & 2 & 6 \\
\hline $\begin{array}{l}\text { availability of a function to tailor available information to } \\
\text { personal needs }\end{array}$ & 5 & 2 & 4 & 1 & 5 \\
\hline simple site design ${ }^{b d}$ & 6 & 1 & 6 & 1 & - \\
\hline clear navigation structure $^{b}$ & 7 & 1 & 2 & 2 & - \\
\hline
\end{tabular}

According to your opinion, which factors determine whether visitors complete questionnaires provided on health related internet sites? 


\begin{tabular}{|c|c|c|c|c|c|c|c|}
\hline \multicolumn{2}{|c|}{ Factora } & \multicolumn{4}{|c|}{ Second round } & \multirow{2}{*}{\multicolumn{2}{|c|}{$\begin{array}{c}\text { Third round } \\
\text { Experts } \\
N=32\end{array}$}} \\
\hline & & \multicolumn{2}{|c|}{$\begin{array}{c}\text { Experts } \\
N=60\end{array}$} & \multicolumn{2}{|c|}{$\begin{array}{c}\text { Users } \\
\mathrm{N}=120\end{array}$} & & \\
\hline & & Mdn & IQD & Mdn & IQD & Mdn & IQD \\
\hline 56. & $\begin{array}{l}\text { provide information on relevance of questionnaire } \\
\text { completion }^{b}\end{array}$ & 6 & 1 & 5 & 1 & - & - \\
\hline 57. & provide incentives for completion & 5 & 2 & 5 & 1 & 5 & 2 \\
\hline 58. & provide information personal benefits of completion $^{b}$ & 6 & 1 & 5 & 1 & - & - \\
\hline 59. & provide a progress bar ${ }^{b d}$ & 6 & 1 & 6 & 1 & - & - \\
\hline 60. & $\begin{array}{l}\text { provide clearly structured questionnaire by using clear } \\
\text { heads and subheads }{ }^{\text {bd }}\end{array}$ & 6 & 1 & 6 & 1 & - & - \\
\hline 61. & $\begin{array}{l}\text { provide guarantee that completion will not result in the } \\
\text { receipt of spam }{ }^{d}\end{array}$ & 6 & 2 & 7 & 1 & 5 & 1 \\
\hline 62. & $\begin{array}{l}\text { provide personalized feedback on questionnaire results } \\
\text { after completion }\end{array}$ & 5 & 2 & 5 & 1 & 5 & 2 \\
\hline 63. & $\begin{array}{l}\text { divide extensive questionnaires into several shorter } \\
\text { parts }\end{array}$ & 5 & 1 & 5 & 2 & - & - \\
\hline 64. & $\begin{array}{l}\text { provide opportunity to stop completion and proceed at } \\
\text { a later point in time }\end{array}$ & 6 & 1 & 6 & 1 & - & - \\
\hline & provide guidance during questionnaire completion & 5 & 2 & 5 & 2 & 5 & 2 \\
\hline 66. & use of original questions ${ }^{d}$ & 4 & 1 & 6 & 1 & - & - \\
\hline
\end{tabular}

What kind of visual aids should be provided on health related internet sites, according to your opinion?
67. provide cartoon
68. provide pictures
69. provide videos to replace written text
70. provide graphical representations of relevant infor- mation
71. refrain from using visual aids
72. provide opportunity for visitors to determine the use of visual aid

What additional services should be provided on health related internet sites, according to your opinion?

\begin{tabular}{|c|c|c|c|c|c|}
\hline $\begin{array}{l}\text { 73. provide a self-monitoring tool to monitor personal } \\
\text { health behavior change }{ }^{b}\end{array}$ & 6 & 1 & 5 & 2 & - \\
\hline 74. provide an ask-the-experts section & 5 & 1 & 5 & 1 & - \\
\hline $\begin{array}{l}\text { provide opportunity to communicate in public with } \\
\text { other visitors, e.g. discussion board, forum }\end{array}$ & 5 & 1 & 4 & 2 & - \\
\hline $\begin{array}{l}\text { provide opportunity to communicate in private with } \\
\text { other visitors, e.g. chat }\end{array}$ & 4 & 1 & 4 & 2 & - \\
\hline provide the opportunity to start a personal blog & 4 & 1 & 4 & 2 & - \\
\hline provide contact information from developers & 5 & 1 & 4 & 1 & - \\
\hline provide information on the working of the site & 5 & 2 & 5 & 1 & 4 \\
\hline provide a privacy statement ${ }^{c}$ & 6 & 2 & 5 & 2 & 6 \\
\hline $\begin{array}{l}\text { provide the opportunity to print or download relevant } \\
\text { information }{ }^{\text {b }}\end{array}$ & 6 & 1 & 5 & 1 & - \\
\hline
\end{tabular}




Factora

Mdn, median score

a Only experts were included in the third round. Statements on which consensus was obtained in the second round were excluded from the third round and results are therefore missing and indicated with a line. The bold values represent the factors on which consensus was obtained (IQD $\leq 1)$.

$b$ indicates items on which consensus was obtained in the second round by experts and which had a median score $\geq 6$.

$c$ indicates items on which consensus was obtained in the third round by experts and which had a median score $\geq 6$.

$d$ indicates items on which consensus was obtained in the second round by potential future users and which had a median score $\geq 6$. 


\title{
APPENDIX 2 - EMAIL PROMPT
}

\section{Beste deelnemer aan mijnGezondGedrag,}

"Is uw huidige leefstijl gezond?"

Een aantal maanden geleden hebt $u$ via de website van mijnGezondGedrag een antwoord gekregen op deze vraag. $U$ hebt toen een persoonlijk gezondheidsoverzicht ontvangen over roken, alcohol, voeding en beweging. Mogelijk bent u ook verder gegaan met ons programma en heeft u over één van deze gedragingen uitgebreide adviezen en tips ontvangen.

\section{Bent $u$ sindsdien gezonder gaan leven?}

Voor een antwoord op deze vraag, kunt $\mathrm{u}$ hier klikken. Wij zullen dan een nieuw persoonlijk gezondheidsoverzicht voor u samenstellen, zodat u uw huidige leefstijl kunt vergelijken met de situatie van 3 maanden geleden.

Is het niet mogelijk om bovenstaande link aan te klikken? Dan adviseren wij u om onderstaande link te kopiëren naar uw internetbrowser:

\section{http://www.mijngezondgedrag.nl/doe-de-test}

Gebruik op de website onderstaande gegevens om in te loggen op uw persoonlijke pagina:

\section{Gebruikersnaam: \\ [LOGIN] \\ Wachtwoord: \\ [PASSWORD]}

\author{
We hopen u snel weer te zien op www.mijnGezondGedrag.nl! \\ Hartelijke groet, \\ Het mijnGezondGedrag-team
}

Dit programma is onderdeel van een onderzoek uitgevoerd door de Universiteit Maastricht. Alle data worden streng vertrouwelijk behandeld en niet doorgegeven aan derden. Neem voor vragen en suggesties gerust contact met ons op via info@mijngezondgedrag.nl 


\section{APPENDIX 3 - STANDARD EMAIL PROMPT}

\section{Welkom terug bij Gezondheid! Limburg}

\section{Beste deelnemer aan Gezondheid! Limburg,}

Onlangs hebt u onze website Gezondheid! Limburg bezocht, waar u een persoonlijk gezondheidsoverzicht hebt ontvangen. Hopelijk hebben onze adviezen u op weg kunnen helpen bij het ontwikkelen van een gezonde leefstijl!

\section{Bent u sindsdien gezonder gaan leven?}

Binnen ons vernieuwde programma kunt $u$ snel zien of $u$ gezonder bent gaan leven en hoe gezond uw leefstijl op dit moment is. Bovendien vindt u er leuke nieuwtjes, tips en adviezen!

Graag nodigen we u uit om ons programma te bezoeken! We hopen u snel weer te zien op www.gezondheidlimburg.n!!

\section{Hartelijke groet,}

Het Gezondheid! Limburg-team

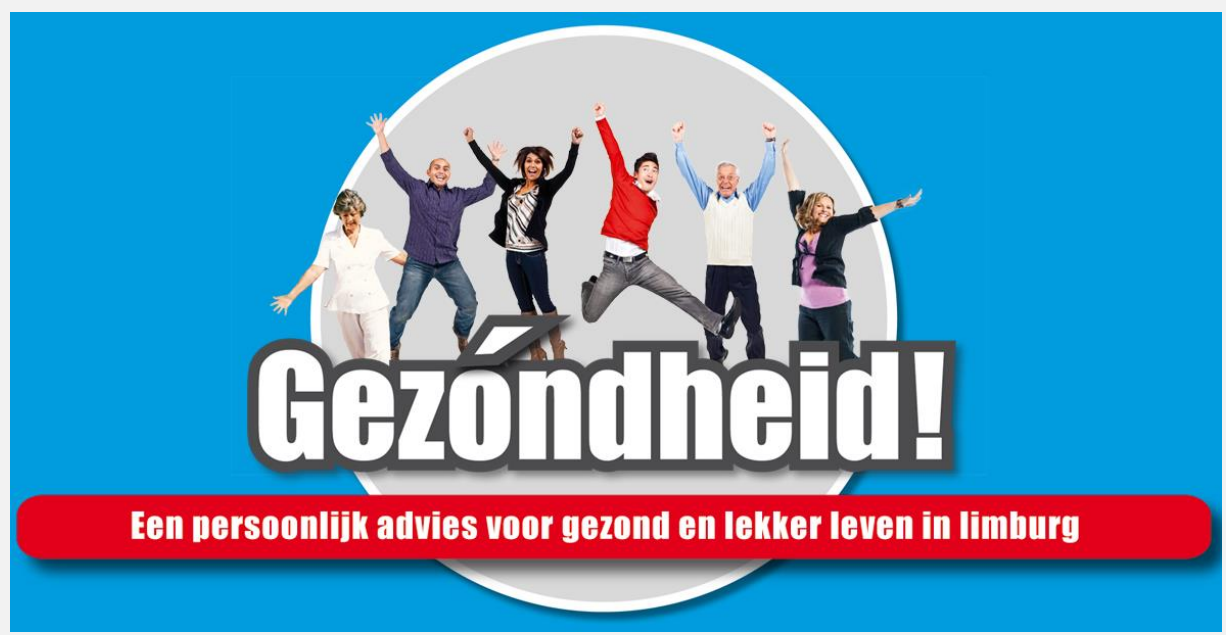

Dit programma is onderdeel van een onderzoek uitgevoerd door de Universiteit Maastricht. Alle data worden streng vertrouwelijk behandeld en niet doorgegeven aan derden. Neem voor vragen en suggesties gerust contact met ons op via gezondheidlimburg@maastrichtuniversity.nl 


\section{APPENDIX 4 - STANDARD+ EMAIL PROMPT}

Welkom terug bij Gezondheid! Limburg

$U$ weet waarschijnlijk dat alle groenten hun eigen seizoen hebben.

Maar weet u ook welke soorten groente het beste verkrijgbaar zijn in het voorjaar? Bezoek nu onze website om meer informatie te krijgen over typische voorjaarsgroenten en recepten!

Beste deelnemer aan Gezondheid! Limburg,

Onlangs hebt u onze website Gezondheid! Limburg bezocht, waar u een persoonlijk gezondheidsoverzicht hebt ontvangen. Hopelijk hebben onze adviezen u op weg kunnen helpen bij het ontwikkelen van een gezonde leefstij!!

Bent u sindsdien gezonder gaan leven?

Binnen ons vernieuwde programma kunt $u$ snel zien of $u$ gezonder bent gaan leven en hoe gezond uw leefstijl op dit moment is. Bovendien vindt u er leuke nieuwtjes, tips en adviezen!

Graag nodigen we u uit om ons programma te bezoeken! We hopen u snel weer te zien op www.gezondheidlimburg.nl!

Hartelijke groet,

Het Gezondheid! Limburg-team

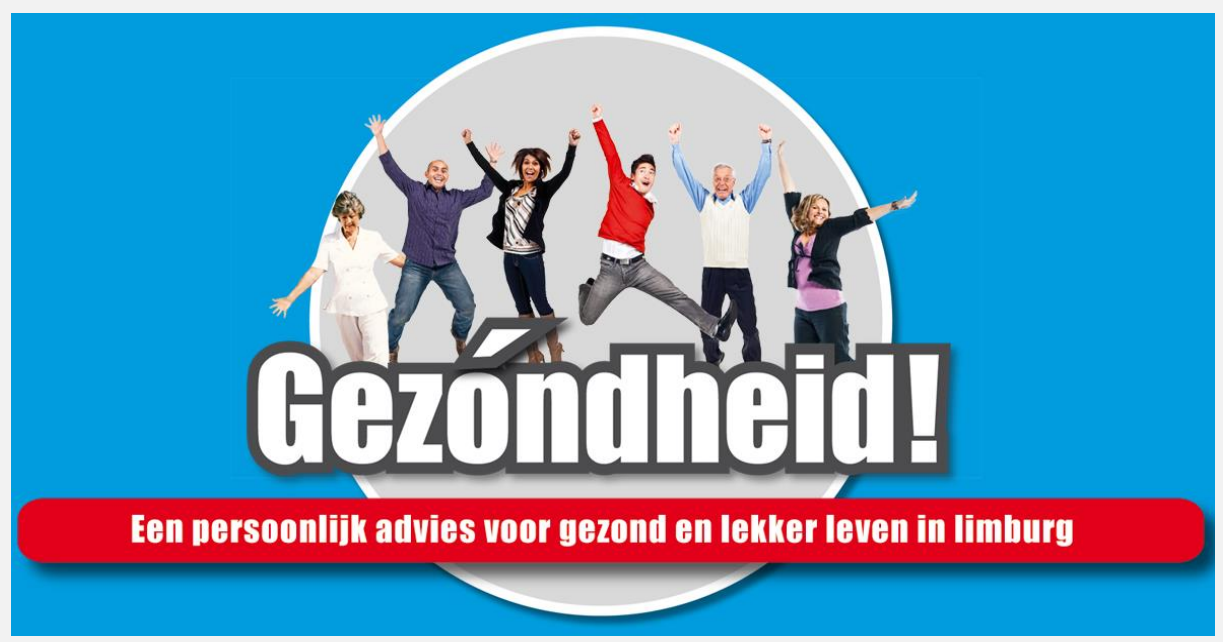

Dit programma is onderdeel van een onderzoek uitgevoerd door de Universiteit Maastricht. Alle data worden streng vertrouwelijk behandeld en niet doorgegeven aan derden. Neem voor vragen en suggesties gerust contact met ons op via gezondheidlimburg@maastrichtuniversity.nl 
Summary 

The Internet offers a good platform for the delivery of lifestyle interventions in terms of reach among the general public, as currently almost $93 \%$ of all Dutch inhabitants has access to the Internet. Even though the empirical support for the effects of Internet-delivered interventions has been demonstrated and is still emerging, reach and use of these interventions is failing to live up to the high expectations. In order to increase public health impact of Internet-delivered interventions, it is imperative to optimize intervention reach and use. The focus of this thesis was on optimizing reach and use of an Internet-delivered computer tailored lifestyle intervention, by emphasising first, prolonged and sustained use. Chapter 1 provides a general introduction to the studies presented in this dissertation. It provides an overview of the background of the studies that were conducted as well as the main aims and research questions.

The first part of this dissertation, aimed at optimizing reach and first use of the intervention, included two chapters. Chapter 2 provides an overview and rationale of the main aims of this dissertation as well as an extensive description of the intervention, consisting of an Internet-delivered computer tailored lifestyle intervention aimed at physical activity, fruit and vegetable intake, smoking cessation and alcohol consumption.

Chapter 3 describes a prospective study in which the effect of a proactive dissemination strategy was examined by studying (1) the absolute number and proportions of intervention users and (2) the adequacy of reach. This strategy entailed embedding of the intervention in an already existing channel; a nationwide survey, the Adult Health Monitor, used by all Dutch Regional Public Health Services (RPHS). This survey is used to periodically assess the general health state of all Dutch adult inhabitants. Findings of this survey are used to outline future health policies at a national, provincial and local level. In total, 41.155 invitees (43\%) participated in the Adult Health 
Monitor of which 16.940 (41\% of all participants) completed the online version. 9.169 participants ( $54 \%$ of the online sample) indicated their interest in the program and 5.168 participants (31\% of the online sample and $56 \%$ of the sample of people interested in participating) paid a first visit to the intervention. Results regarding adequacy of reach indicated that intervention users were more likely to be male, older and higher educated, with a healthier lifestyle and a lower BMI compared to non-users. With one out of three online Adult Health Monitor participants actually using the intervention, the employed proactive dissemination strategy succeeded in ensuring relatively high levels of reach. With regard to adequacy of reach, results indicated that reach among at-risk individuals (e.g. low socioeconomic status and unhealthy lifestyle) was lower compared to low-risk individuals.

The second part of this dissertation aimed at optimizing prolonged use of the intervention included two chapters. Chapter 4 describes a three-round Delphi study to identify factors associated with optimal development of health-related websites, as well as the extent to which experts and potential future users agreed on the importance of these factors. Experts and potential future users both agreed that websites should provide information on how to attain a healthy lifestyle. Furthermore, the website needs to be easy to use, with a clear structure, easy log-in procedure and simple design. Finally, in order to stimulate questionnaire completion, websites should display a progress bar and provide clearly structured questionnaires, which can be completed in parts. Development of the Internet-delivered intervention content and its online environment was guided by the results from this Delphi study.

Chapter 5 describes a study that examined prolonged use of the intervention by studying module initiation and completion as well as predictors of initiation and completion ( $\mathrm{N}=3448$ ). Furthermore, the effect of using a periodic email prompt to encourage re-use of the intervention was tested in a randomized control trial. Results indicated that $61 \%$ of all intervention users initiated one of the modules. In comparison with people that refrained from initiating the modules, initiators were more likely to be male, of older age, employed and had a lower income. Furthermore, with regard to their health behaviour, module initiators were more likely to have a higher body mass index and an unhealthier lifestyle. $62 \%$ of all initiators actually completed the module, with older participants and participants with a higher income and a relatively healthy lifestyle being more likely to complete the module. Positive effects of the email prompt were found on sustained use of the intervention. People that received an additional email prompting them to revisit the program were more likely to revisit the program compared to people that did not receive an additional prompt ( $6 \%$ vs. $0.2 \%$ ). Based on these findings we can conclude that the program was able to persuade those people who benefit most from online life-style interventions, e.g. people with a 
lower income and unhealthier lifestyle, to initiate one of the CT modules. How ever, these people tended to disengage from the intervention before actually finishing these modules. Furthermore, sending periodic email prompts significantly increased revisits to the program. Though promising, this effect was modest and needs to be further examined, in order to maximize the potential of periodic email prompting.

With this aim, the third and final part of this dissertation focussed at optimizing sustained use of the intervention. Chapter 6 describes a $2 \times 3$ design used to test the effect prompt content and prompt timing. The first version of the prompt contained an invitation to re-use the intervention, whereas a second version was complemented with a message alerting people to new content added to the intervention website. Participants received an email prompt after two, four or six weeks. In addition to these six experimental conditions, a control no-prompt condition was included. Results indicated that sending a prompt two weeks after the first visit was more effective compared with using a longer time period. This effect not only referred to clicking on the URL, but also to the proportion of log-ins to the program. Secondly, a trend was observed indicating that adding a preview of new website content to a standard prompt increased its effectiveness in persuading people to log in to the program. Finally, sending a prompt with additional content after a two-week period significantly increased program log-ins compared to using a reactive approach in which no additional prompts were used. The findings of this study underline the importance of sending an email prompt relatively shortly after a first visit to the program.

Chapter 7 provides an overview and integrated discussion of the results of the presented studies. In addition important considerations regarding these results are addressed and recommendations for future research and practice are provided. 
Samenvatting 

Het internet vormt een veelbelovend platform voor het aanbieden van leefstijl interventies aan de algemene bevolking. Aangezien in Nederland momenteel bijna 93\% van de bevolking toegang heeft tot het internet, is het potentiele bereik van dit medium behoorlijk groot. Ondanks dat het empirische bewijs voor het effect van deze online leefstijlinterventies veelvuldig geleverd is en nog groeit, voldoet het daadwerkelijke bereik van deze interventies vooralsnog niet aan de hoge verwachtingen. Om de impact van effectieve leefstijlinterventies op de volksgezondheid te vergroten, is het van het grootste belang om het bereik en het gebruik van deze interventies te optimaliseren.

De focus van dit proefschrift lag op het optimaliseren van bereik en gebruik van een leefstijlinterventie die werd aangeboden via het internet. Hierbij werd de nadruk gelegd op drie verschillende vormen van gebruik; eerste gebruik, aanhoudend gebruik en herhaald gebruik. Hoofdstuk 1 geeft een algemene introductie van de studies die in dit proefschrift worden gepresenteerd. Het biedt een overzicht van de achtergrond en rationale voor de studies die zijn uitgevoerd, evenals een beschrijving van de belangrijkste onderzoeksvragen.

Het eerste deel van dit proefschrift, gericht op het optimaliseren van bereik en eerste gebruik van een leefstijlinterventie, bestaat uit twee hoofdstukken. Hoofdstuk 2 biedt een uitgebreid overzicht van de rationale en de belangrijkste doelen van dit proefschrift. Verder biedt dit hoofdstuk een uitgebreide beschrijving van de gebruikte online leefstijlinterventie, die bestond uit een interventie aangeboden gericht op fysieke activiteit, fruit en groente consumptie, rookgedrag en alcoholinname.

In hoofdstuk 3 wordt een prospectieve studie beschreven waarin het effect van een proactieve disseminatiestrategie is bestudeerd. De focus lag hierbij op het in kaart brengen van (1) het absolute aantal en de proporties van interventiegebruikers en (2) de adequaatheid van bereik. Deze proactieve strategie hield in dat de leefstijlinterventie werd ingebed in een bestaand kanaal: de Gezondheidsenquête, gebruikt door alle Gemeenschappelijke Gezondheidsdiensten (GGD) in Nederland. Deze Gezondheids- 
enquête is een periodieke meting die wordt uitgevoerd om de Gezondheid van de Nederlandse bevolking in kaart te brengen. De resultaten van de enquête worden vervolgens gebruikt als input bij de ontwikkeling van toekomstig gezondheidsbeleid op een nationaal, provinciaal en regionaal niveau. De huidige online leefstijlinterventie werd geïncorporeerd in de Gezondheidsenquête van de verschillende GGD'en (GGD Brabant-Zuidoost, GGD Hart voor Brabant, GGD West-Brabant en GGD Zeeland) in de Nederlandse provincies Noord-Brabant en Zeeland. De resultaten van de enquête worden vervolgens gebruikt als input bij de ontwikkeling van toekomstig gezondheidsbeleid op een nationaal, provinciaal en regionaal niveau. In totaal hebben 41.155 genodigden (43\%) deelgenomen aan deze Gezondheidsenquête. Van deze deelnemers, namen 16.940 deelnemers (41\% van alle deelnemers) deel middels een enquête die werd aangeboden via het internet. Het merendeel van de internet-deelnemers (9.169; (54\%) gaf aan geïnteresseerd te zijn in deelname aan de leefstijlinterventie. Deze deelnemers werden vervolgens uitgenodigd om de interventie te bezoeken. Dit resulteerde in een totaal aantal van 5.168 deelnemers (31\% van alle deelnemers via internet en $56 \%$ van alle geïnteresseerde deelnemers) dat daadwerkelijk gehoor gaf aan deze uitnodiging door de interventie een eerste keer te gebruiken. De resultaten betreffende de adequaatheid van het bereik laten zien dat interventiegebruikers veelal mannen waren, met een hogere leeftijd en opleiding. Verder hadden deze deelnemers een gezondere leefstijl en een lagere body mass index (BMI) in vergelijking met deelnemers die geen gebruik maakten van de interventie. Aangezien 1 van de drie online deelnemers gebruik maakte van de leefstijlinterventie, kon de gebruikte proactieve disseminatiestrategie als succesvol worden beschouwd in het verhogen van het bereik van de leefstijlinterventie. Wanneer we kijken naar de adequaatheid van het bereik valt op dat het bereik onder risicogroepen (bijv. deelnemers met een lagere socio-economische achtergrond en een relatief ongezonde leefstijl) lager lag dan het bereik onder groepen met een lager risico.

Het tweede deel van dit proefschrift was gericht op het optimaliseren van aanhoudend gebruik van de interventie. Hoofdstuk 4 beschrijft een Delphi studie die werd uitgevoerd om in kaart te brengen welke factoren geassocieerd zijn met een optimale ontwikkeling van websites over gezondheid. Daarnaast werd ook de mate waarin experts en toekomstige gebruikers overeenstemming bereiken over het belang van deze factoren onderzocht. Zowel experts als toekomstige gebruikers waren het eens over het belang van een aanzienlijk aantal factoren. Zo vonden beide groepen dat deze websites informatie moesten bieden over de wijze warop een gezonde leefstijl verkregen kan worden. Daarnaast was het ook van belang dat de website gemakkelijk in het gebruik is en een overzichtelijke structuur heeft met een eenvoudige registratieprocedure en een simpel design. Beiden groepen waren van mening dat verschil- 
lende factoren het invullen van vragenlijsten die worden aangeboden op website kunnen bevorderen. Deze factoren hadden betrekking op het aanbieden van een voortgangsbalk en op het aanbieden van goed gestructureerde vragenlijsten, die in delen ingevuld kunnen worden.

In hoofdstuk 5 worden de resultaten beschreven van een studie naar aanhoudend gebruik van de leefstijlinterventie. De focus lag hierbij op het bestuderen van de mate van initiatie en voltooiing van een interventiemodule, evenals het bepalen van belang rijke voorspellers van initiatie en voltooiing $(\mathrm{N}=3.448)$. Daarnaast werd het effect van het sturen van een periodieke emailprompt op het herhaald gebruik van de interventie bestudeerd in een gerandomiseerd gecontroleerd experiment (RCT). De resultaten van de studie in dit hoofdstuk laten zien dat 61\% van alle deelnemers die een eerste bezoek brachten aan de interventie daadwerkelijk een van de beschikbare gedragsmodules initieerden. In vergelijking met deelnemers die er voor kozen om niet aan een van de modules te beginnen, waren deze deelnemers veelal van het mannelijke geslacht en hadden zij een relatief hogere leeftijd. Ook hadden deze deelnemers vaker een baan en een lager inkomen. Als we kijken naar het gezondheidsgedrag van deelnemers die een module initieerden, valt het op dat deze deelnemers een hogere BMI en relatief ongezondere leefstijl hadden in vergelijking met deelnemers die afzagen van het gebruiken van een van de modules. $62 \%$ van alle deelnemers die een module initieerde, rondde deze module ook daadwerkelijk af. Wanneer we kijken naar de eigenschappen van de deelnemers die de modules afrondden, valt te zien dat dit vooral de deelnemers zijn met een hogere leeftijd, een hoger inkomen en een relatief gezondere leefstijl. In het licht van deze resultaten kunnen we concluderen dat de interventie in staat was om juist die deelnemers te trekken die de meeste baat kunnen hebben bij een leefstijlinterventie, aangezien deelnemers die de module initieerden veelal een lager inkomen en een ongezondere leefstijl hadden. Echter, juist deze mensen bleken deze modules veelal niet te voltooien. Daarnaast bleek het versturen van een periodieke email prompt een positief effect te hebben op herhaald gebruik van de interventie. Deelnemers die drie maanden na gebruik van de interventie een email prompt ontvingen, waren meer geneigd om de interventie opnieuw te gebruiken in vergelijking met deelnemers die geen additionele prompt ontvingen (6\% vs. $0.2 \%)$. Hoewel veelbelovend, was dit effect beperkt. Toekomstig onderzoek dat ingaat op het verfijnen van deze email prompts is dan ook van belang om het effect te optimaliseren.

Met bovenstaande aanbeveling voor toekomstig onderzoek in gedachten, was het derde en laatste deel van dit proefschrift gericht op het optimaliseren van herhaald gebruik van de leefstijlinterventie. Hoofdstuk 6 beschrijft een gerandomiseerd RCT met een $2 \times 3$ design dat werd gebruikt om het effect van prompt inhoud en timing 
te testen. Hiertoe werden twee verschillende versies van een prompt gebruikt. De eerste versie van deze prompt bevatte enkel een uitnodiging om de interventie opnieuw te bezoeken, terwijl de tweede versie naast deze informatie ook een boodschap bevatte die deelnemers wees op nieuwe informatie die was toegevoegd aan de interventiewebsite. Deelnemers in de experimentele condities ontvingen een van beide prompts na twee, vier of na zes weken. Deelnemers in de controle conditie ontvingen geen additionele email prompt. De resultaten van deze studie laten zien dat het sturen van een email prompt na twee weken een significant groter effect had dan het sturen van een prompt na vier of zes weken. Dit effect had niet alleen betrekking op het klikken URL in de email, maar ook op het daadwerkelijk inloggen in de interventie. Ten tweede werd er een trend aangetoond, duidend op een positief effect van het toevoegen van een boodschap over nieuwe inhoud op de website. Deelnemers die een prompt met deze boodschap ontvingen waren vaker geneigd om in te loggen in de interventie dan deelnemers die deze informatie niet ontvingen. Tenslotte werd aangetoond dat de combinatie van snelle timing ( 2 weken) en nieuwe inhoud van een email prompt tot een significant hoger aantal log-ins in de interventie leiden in vergelijking met de controlegroep (geen email prompts). Deze studie toont eens te meer aan dat het gebruik van periodieke email prompts een belangrijke rol kan spelen bij het bevorderen van herhaald gebruik van interventies.

Hoofdstuk 7 biedt een overzicht en een geïntegreerde discussie van de resultaten verkregen uit de verschillende studies. In aanvulling op deze discussie worden in dit hoofdstuk ook belangrijke overwegingen besproken in het licht waarvan de resultaten gepresenteerd in dit proefschrift geïnterpreteerd dienen te worden. Tenslotte biedt dit hoofdstuk aanbevelingen voor zowel wetenschappelijk onderzoek als de praktijk van gezondheidsbevordering. 
Curriculum vitae 



\section{CURRICULUM VITAE}

\section{Biography}

Francine Schneider was born in Kerkrade, in the southern part of the Netherlands, on May $3^{\text {rd }}$ 1982. After she graduated from high school (College Rolduc, Kerkrade), she studied psychology at Maastricht University, Maastricht. Here she received her Bachelor's (2007, Cognitive Psychology) and Master's degree (2008, Experimental Health Psychology). While studying for her degree she worked as a student

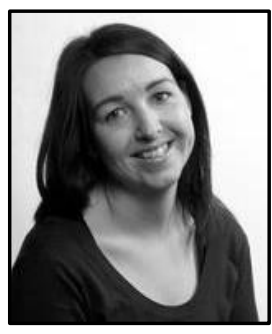
assistant at the Department of Clinical Psychological Science. In 2008, she started working at the Department of Health Promotion at Maastricht University, to conduct the studies that are presented in the dissertation. As of August 2012, she is working as a postdoctoral researcher at this department support by a ZonMw-grant entitled 'Enhancing reach of evidence-based e-mental health courses by using the Adult and Senior Health Monitor environment: a pilot test'.

\section{Publications}

d.d. 26-11-2013

Publications from this thesis

Schneider, F., van Osch, L., Kremers, S.P.J., Schulz, D.N., van Adrichem, M.J.G., \& de Vries, H. (2011). Optimizing diffusion of an online computer tailored lifestyle program: a study protocol. BMC Public Health, 11, 480.

Schneider, F., van Osch, L., \& de Vries, H. (2012). Identifying factors for optimal development of health-related websites: a Delphi study among experts and potential future users. Journal of Medical Internet Research, 14, e18.

Schneider, F., van Osch, L., Schulz, D.N., Kremers, S.P.J., \& de Vries, H. (2012). The influence of user characteristics and a proactive diffusion strategy on depth of use of an Internet-delivered computer tailored lifestyle program. Journal of Medical Internet Research, 14, e40.

Schneider, F., de Vries, H., Candel, M., van de Kar, A., \& van Osch, L. (2013). Periodic Email prompts to re-use an Internet-delivered computer tailored lifestyle program: Influence of Prompt Content and Timing. Journal of Medical Internet Research, 15, e23.

Schneider, F., Schulz, D. N., Pouwels, L. H., de Vries, H., van Osch, L. (2013). The use of a proactive dissemination strategy to optimize reach of an internet-delivered 
computer tailored lifestyle intervention. BMC Public Health, 13, 721.

\section{Other publications}

Alberts, H. , Schneider, F. \& Martijn, C. (2008). Efficiënt omgaan met negatieve emoties: het accepteren van negatieve emoties kost minder energie dan het onderdrukken ervan. In R. Custers, B. Beersma, H. van den Berg, F. Harinck \& M. van Zomderen (Eds.), Jaarboek Sociale Psychologie. Groningen: Aspo Pers.

Schulz, D.N., Kremers, S.P.J., van Osch, L., Schneider, F., van Adrichem, M.J.G., \& de Vries, H. (2011). Testing a Dutch web-based tailored lifestyle program among adults: a study protocol. BMC Public Health, 11, 108.

Schulz, D.N., Schneider, F., de Vries, H., van Osch, L., van Nierop, P.W., \& Kremers, S.P. (2012). Program completion of a web-based tailored lifestyle intervention for adults: differences between a sequential and a simultaneous approach. Journal of Medical Internet Research, 14, e26.

Alberts, H.J., Schneider, F., \& Martijn, C. (2012). Dealing efficiently with emotions:

Acceptance-coping with negative emotions requires fewer resources than suppression. Cognition and Emotion, 26, 863-870.

Schulz, D.N., Kremers, S.P., Vandelanotte, C., van Adrichem, M. J. G., Schneider, F., Candel, M. J. J. M., \& de Vries, H. Effects of a web-based tailored multiple lifestyle intervention for adults: A two-year randomized controlled trial comparing sequential and simultaneous delivery modes, submitted. 
Dankwoord 


\section{(CO) PROMOTER}

Hein, bedankt voor je begeleiding, je inbreng, onze discussies en de kansen die je me geboden hebt. Je stimuleert me altijd om tot het uiterste te gaan. Liesbeth, je kritische blik en je oog voor detail zijn werkelijk bewonderenswaardig. Door jou wist ik steeds het beste uit mezelf te halen. Bedankt voor alles!

\section{DE GGD}

Graag wil ik alle deelnemende GGD'en bedanken voor de fijne samenwerking gedurende dit project; GGD BrabantZuidoost, GGD Hart voor Brabant, GGD West-Brabant, GGD Zeeland, GGD Zuid Limburg en GGD Limburg-Noord. Een speciaal woord van dank voor Angélique, Peter en Klaas Jan.

\section{HET TEAM}

Omdat het geheel zeker meer is dan de som der delen: Mathieu en Daniela, het was heel leuk om samen met jullie aan dit project te werken. Veel dank voor alles! Stef, heel erg bedankt voor je begeleiding vanaf de zijlijn. Kim, bedankt voor al je hulp (en gezelligheid!).

\section{COLLEGA'S}

Als 'gezelligheidsmens' is het heel fijn om leuke collega's om je heen te hebben. Ik had dat geluk. Collega's, bedankt voor de gezellige sfeer, de goede hulp en raad, de inhoudelijke discussies en niet te vergeten de leuke uitstapjes! 


\section{COLLEGA EN MEER}

Eline, Judith, Eva, Karen en Leonieke - de Goulash: Het was geweldig om samen met jullie aan dit avontuur te beginnen. Ik vind het heel leuk dat we elkaar niet uit het oog zijn verloren. Eline, ik had geen andere kamergenoot willen hebben. Bedankt voor alles. Erg fijn dat jij mijn paranimf bent!

\section{EN VOORAL...}

Rik, lief. Omdat iedere dag met jou een geweldig feest is!

\section{FAMILIE}

Pap, mam, Rob; jullie zijn mijn ontzettend fijne thuisfront. Heel erg bedankt voor alles. En natuurlijk voor nog veel meer! 
